\title{
Ultrafast Spectroscopy for Printable Photovoltaics
}

\author{
by \\ Silvina Norma Pugliese
}

A thesis submitted to Victoria University of Wellington in fulfilment of the requirements for the degree of Doctor of Philosophy

Victoria University of Wellington 2020 


\section{Abstract}

The development of efficient and low cost photovoltaic technologies is key to a more sustainable energy pathway for future generations. Research efforts aimed at improving the performance of organic photovoltaic (OPV) materials have resulted in a continuous growth in power conversion efficiency (PCE) over time, with a recent maximum PCE value of $18.22 \%$ in a single bulk heterojunction device. However, further improved efficiency, stability and cost reduction are required in order for OPVs to succeed in the market.

To produce better performing OPV devices in a rational way, it is necessary to understand the relationships between material properties (e.g. energy levels, recombination rates, charge carrier mobilities) and the photovoltaic parameters. This requires combining different fundamental techniques, such as spectroscopic, electrical and structural studies of the materials. In this thesis work we contribute to the understanding of the mechanisms of charge photo-current generation in OPV layers by using transient absorption spectroscopy (TAS) to directly measure the fate of the photo-excited species created upon light absorption. In particular, we contribute to the understanding of the dynamical properties of tightly bound, interfacial charge-transfer (CT) states at the donor:acceptor heterojunction. We disentangle the contributions from individual transient species to the overall TAS signal via the soft-modelling algorithm known as Multivariate Curve Resolution by Alternating Least Squares (MCR-ALS), and we use simple 
kinetic models to retrieve associated kinetic rates.

Our first study explores the photo-physics of a family of polymers derived from the low-band-gap alternating copolymer PTBT where the sulphur atom in the thiadiazole unit was substituted with oxygen or selenium. The literature shows that replacing a single atom in the donor or acceptor unit of a polymer donor can cause large changes in the photovoltaic parameters, which cannot be explained considering only the variations in the optical band-gap. Opposite results have been reported on systems where a sulfur atom is replaced by selenium, and spectroscopic studies were lacking. Our TAS results on PTBO and PTBSe systems explain the superior photovoltaic performance of the original sulfur-containing variant PTBT, highlighting the low tolerance of these materials to backbone substitutions. In both PTBO and PTBSe systems, we identify strong recombination of geminate CT pairs as the major limiting factor of the Jsc and FF photovoltaic parameters. This is attributed to unfavourable electronic and conformational properties at the donor:acceptor interface. In the particular case of PTBSe:PC61BM, the recombination pathway of CT states with triplet character into the triplet exciton manifold is facilitated by the heavy atom effect, in addition to a highly intermixed morphology.

Our second study comprises the spectroscopic comparison between fullerene and nonfullerene (NFA) OPV layers. The PCE of OPV devices was reaching a plateau in past years, which was overcomed thanks to the development of high efficiency NFA acceptors. Here, we compare charge generation and recombination between three systems featuring the same polymer donor PPDT2FBT matched with three different acceptors, namely the fullerene acceptor PC70BM, the small molecule nonfullerene acceptor NIDCS-HO and the polymeric acceptor N2200. Our results provide insight on the processes that 
limit the performance of each device, showing that small molecule NFA are promising acceptors, since morphology and disorder, the factors that we have found to be limiting the device performance, could potentially be tuned for the development of more efficient materials. For the all-polymer device based on the N2200 acceptor, we find that both geminate and nongeminate recombination are limiting the photovoltaic performance.

Lastly, we investigate charge carrier dynamics in a series of solar devices composed predominantly of C60 and small amounts of organic small molecule donors, where their CT state energies are systematically varied. The well-defined microstructure in low-donor-content OPV blends makes it easier to correlate macroscopic properties to molecular parameters. Our results, in combination with time-delayed collection field (TDCF), and external quantum efficiency measurements (EQE) measurements at different bias performed by our collaborators, allow us to identify geminate recombination as the major loss channel. We find that the dynamics of the CT decay are connected to the CT state energy via the energy-gap law. In this way, the energy of the CT state is identified as the main parameter determining the efficiency of photocurrent generation in these morphologically well-defined donor:acceptor blends.

Overall, the contributions in this thesis work demonstrate how TAS measurements can provide valuable information to construct a comprehensive picture of the underpinning mechanisms of charge photo-current generation in OPV layers, in particular by isolating the dynamical properties of interfacial chargetransfer (CT) states at the donor:acceptor heterojunction via modelling. 


\section{Dedication}

To my wonderful daughter, Aurelia. 


\section{Acknowledgements}

I would like to begin this work by extending my gratitude to people and institutions who have made it possible.

First and foremost, I would like to thank my supervisor Justin Hodgkiss, for the opportunity to do research in his lab, for always finding the time to discuss about research questions, and for being so kind. Not all supervisors are understanding when a student has a baby during the $\mathrm{PhD}$ candidature. I am forever grateful for that.

I would like to thank Petrik Galvosas, Patricia Stein and Kelly Atherton for having listened to me in difficult times within the academic environment, and for taking me seriously. I could not have completed this thesis without their support.

I would like to extend a special thanks to prof. Dieter Neher. It was a huge privilege to have collaborated with his group and I greatly appreciated each communication.

I would also like to thank prof. Han Yang Hoo and prof. Jin Young Kim for the valuable communications, for providing photovoltaic materials for this work and for being so generous when I visited their labs in South Korea.

From Justin Hodgkiss's group, I would like to thank Ronnie for sharing his 
enthusiasm for physics, to Mike and Clement, for offering help with the preparation of samples when I was pregnant (I am really grateful for that!), and to Sreelakshmi, for her patience and help fine tuning the laser beams for the experiments. Thom, Clement, Shalini, Parth, Vaibhav, Yuta and Loc, you have been amazing people to be around. And Thom, thank you so much for your kindness and for giving me very clever and creative advice about the thesis writing process.

I would also like to thank my friends outside academia. Thank you so much Lorraine, Zlata and Irina, sharing time with you always lifts my spirit! A special thanks goes to Lorraine, for staying in contact with me during the isolating times of the thesis writing.

A special thanks goes to my family. To my parents, for their continuous care and support and for having made the big effort of visiting me in Wellington twice. To my wonderful daughter, Aurelia, for bringing so much love and joy to my life, more than I could have ever imagined! And to Richard, for his loving support and taking care of me and Aurelia.

Last but not least, I would like to thank the Ministry of Business, Innovation and Employment and the MacDiarmid Institute for funding my scholarship and for additional support that has allowed me to attend multiple scientific events. 


\section{Contents}

$\begin{array}{llr}1 & \text { Introduction } & 13\end{array}$

1.1 Motivation . . . . . . . . . . . . . . . . . 13

1.1.1 Mitigating the effects of climate change . . . . . . . 13

1.1.2 The scale of the solar resource $\ldots \ldots \ldots \ldots$

1.1.3 Advantages of organic photovoltaic devices . . . . . . . 16

1.2 Outline of this thesis $\ldots \ldots \ldots \ldots \ldots$

2 The Theory of Organic Photovoltaic Devices 18

2.1 Organic semiconductors . . . . . . . . . . . . . . . 18

2.2 Electronic and optical processes . . . . . . . . . . . . 21

2.3 The organic photovoltaic device . . . . . . . . . . . . . 29

2.3.1 From excitons to extractable charge carriers . . . . . . . 34

2.3.2 Photovoltaic parameters . . . . . . . . . . . . . 42

2.4 Recombination losses . . . . . . . . . . . . . . . . . . 44

2.4 .1 Exciton decay $\ldots \ldots \ldots \ldots \ldots$. . . . . . . . . . 44

2.4 .2 Geminate recombination . . . . . . . . . . . 45

2.4 .3 Nongeminate recombination . . . . . . . . . . . . 46

2.5 Research questions addressed in this thesis $\ldots \ldots \ldots \ldots$

3 Experimental Methods and Data Analysis $\quad 52$

3.1 Sample preparation . . . . . . . . . . . . . . . 52 
3.2 UV-Vis absorption spectroscopy $\ldots \ldots \ldots \ldots$. . . . . . . . 53

3.3 Transient absorption spectroscopy . . . . . . . . . . . . . . . 54

3.4 Multivariate curve resolution . . . . . . . . . . . . . . . 62

4 How atom substitution affects the photophysics of low-bandgap copolymers for OPV cells

4.1 Overview . . . . . . . . . . . . . . . . 67

4.2 Background . . . . . . . . . . . . . . . . 69

4.2.1 Single-atom substitution in low-band-gap copolymers . . 69

4.2 .2 Previous study on РТВТ . . . . . . . . . . . 70

4.2.3 The role of spin in organic photovoltaic devices . . . . . . 72

4.3 Results and discussion . . . . . . . . . . . . . . . . 75

4.3.1 Device characteristics . . . . . . . . . . 75

4.3.2 UV-Vis steady state absorption . . . . . . . . . 76

4.3.3 Transient absorption spectroscopy $\ldots \ldots \ldots$. . . . 77

4.3.4 Neat PTBO and PTBO:PC ${ }_{61} \mathrm{BM} \ldots \ldots \ldots \ldots$

4.3.5 Neat PTBSe and PTBSe:PC ${ }_{61} \mathrm{BM} \ldots \ldots \ldots$

4.4 Conclusions . . . . . . . . . . . . . . . . . . . . 94

5 Comparative transient absorption spectroscopy of fullerene and nonfullerene organic photovoltaic cells $\quad 96$

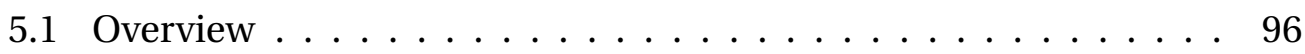

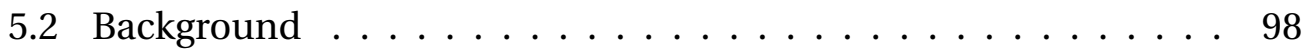

5.2.1 The donor material PPDT2FBT . . . . . . . . . . . . 98

5.2.2 The small molecule electron acceptor NIDCS-HO . . . . . 100

5.2.3 The polymer electron acceptor N2200 . . . . . . . . 100

5.2.4 Fullerene vs. nonfullerene organic photovoltaics . . . . . 105

5.3 Results and discussions $\ldots \ldots \ldots$. . . . . . . . . . . 106 
5.3.1 Device characteristics . . . . . . . . . . . . 106

5.3.2 UV-Vis steady state absorption . . . . . . . . . . . . 107

5.3.3 Transient absorption spectroscopy . . . . . . . . . 108

5.3.4 Excited states in the neat materials . . . . . . . . . . 109

5.3.5 Donor:acceptor blend films - Assignment of the transient spectral features . . . . . . . . . . . . . 113

5.3.6 Donor:acceptor blend films - Charge generation . . . . . 121

5.3.7 Donor:acceptor blend films - Charge recombination . . . 125

5.4 Conclusions . . . . . . . . . . . . . . . . . . . . . . . 134

6 Charge Photogeneration in Low-Donor-Content Organic Photovoltaic Blends $\quad 136$

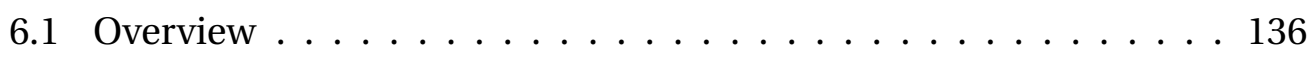

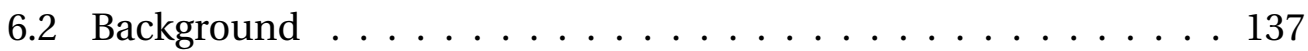

6.2.1 Low-donor-content organic photovoltaic blends . . . . . 137

6.2 .2 Nonradiative voltage losses . . . . . . . . . . . 138

6.3 Results and discussion . . . . . . . . . . . . . . . . . . . 140

6.3.1 Device performance versus open-circuit voltage . . . . . 141

6.3.2 Bias and photon energy dependence of free charge generation143

6.3.3 Transient absorption spectroscopy . . . . . . . . . . . 145

6.3.4 Spectral assignments . . . . . . . . . . . . . . . . 145

6.3.5 Charge carrier dynamics . . . . . . . . . . . . . 150

6.4 Conclusions . . . . . . . . . . . . . . . . . . . 157

$\begin{array}{lll}7 & \text { Conclusions } & 160\end{array}$

$\begin{array}{ll}\text { A Sample Preparation } & 163\end{array}$

A.1 Preparation of the PTBO and PTBSe solutions . . . . . . . . . 163

A.2 Preparation of the PPDT2FBT:PC ${ }_{70} \mathrm{BM}$ solution $\ldots \ldots \ldots$. . . . . 164 
A.3 Preparation of the PPDT2FBT:NIDCS-HO solution . . . . . . . . . 164

A.4 Preparation of PPDT2FBT:N2200 solution . . . . . . . . . . . . 164 


\title{
List of Abbreviations
}

\author{
ALS Alternating least squares \\ BHJ Bulk heterojunction \\ BMR Bimolecular recombination \\ CT Charge transfer \\ DOS Density of states \\ ECA Exciton-charge annihilation \\ EFA Evolving factor analysis \\ EGE External generation efficiency \\ EQE External quantum efficiency \\ ET Electron transfer \\ FC Franck-Condon factor \\ FF Fill factor \\ FWHM Full width half maximum \\ GSB Ground state bleach \\ HOMO Highest-occupied molecular orbital \\ HT Hole transfer \\ IC Internal conversion \\ ICS Intersystem crossing \\ IPCC Intergovernmental panel on climate change \\ IQE Internal quantum efficiency
}


$J_{s c} \quad$ Short-circuit current

LCAO Linear combination of atomic orbitals

LUMO Lowest-unoccupied molecular orbital

MCR Multivariate curve resolution

NFA Nonfullerene acceptor

OPA Optical parametric amplifier

OPV Organic Photovoltaic

PCE Power conversion efficiency

PIA Photoinduced absorption

PV Photovoltaic

SC Separated charges

SE Stimulated emission

SVD Singluar value decomposition

TAS Transient absorption spectroscopy

TDCF Time delayed collection field

UV-Vis Ultraviolet-visible spectroscopy

$V_{o c} \quad$ Open-circuit voltage

VR Vibrational relaxation 


\section{Chapter 1}

\section{Introduction}

\subsection{Motivation}

\subsubsection{Mitigating the effects of climate change}

Historically, nonrenewable fossil fuels such as coal, oil and gas have been the major sources of human energy consumpsion, providing electricity, heat, transportation, and energy for a wide range of purposes in the industrial sector. When these fuels are burned, carbon dioxide and other greenhouse and polluting gasses are released into the atmosphere, making them the primary contributors to global warming and climate change $[1,2]$. Today, the scientific evidence for human-induced climate change is unequivocal [3].

In December 2015, 195 nations committed to the Paris Agreement to address the threats of climate change. The aim of the Paris agreement was to strengthen the global response and keep the global temperature rise within this century well below 2 degrees Celsius above pre-industrial levels (the time period between 1850 and 1900). The target limit for the temperature increase above pre-industrial levels was set to 1.5 degrees Celsius [4]. 
To assess the impacts of global warming on our planet in various scenarios, the Intergovernmental Panel on Climate Change (IPCC) released a Special Report on Global Warming of 1.5 Degrees Celsius [5]. The report was written by 91 authors and review editors from 40 countries along with 133 contributing authors and cites more than 6000 scientific references [6]. Figure 1.1, extracted from the IPCC report, shows the observed monthly global mean surface temperature change (grey line up to 2017) and the estimated anthropogenic global warming (solid orange line up to 2017, with orange shading indicating assessed likely range). The orange dashed arrow and horizontal orange error bar show respectively the central estimate and likely range of the time at which 1.5 degrees Celsius is reached if the current rate of warming continues. We can see in Figure 1.1 that global warming is likely to reach 1.5 degrees Celsius above pre-industrial levels sometime between 2030 and 2052, with a best estimate of around 2040 [6] 


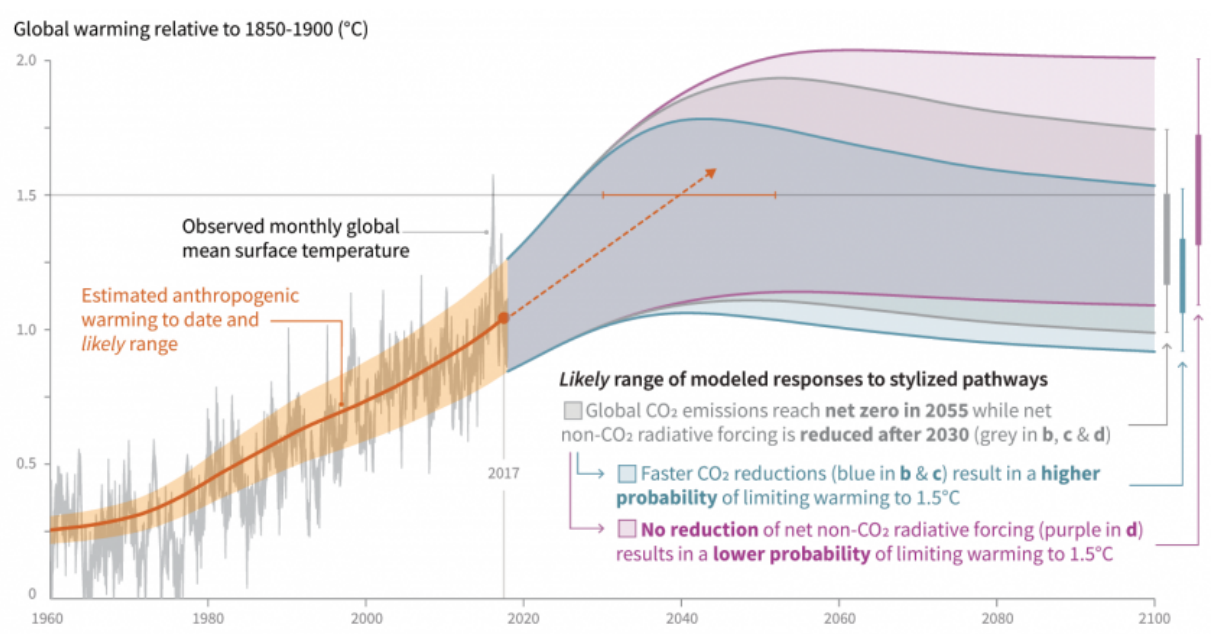

Figure 1.1: Monthly global mean surface temperature change (grey line up to 2017) and the estimated anthropogenic global warming (solid orange line up to 2017, with orange shading indicating assessed likely range). Orange dashed arrow and horizontal orange error bar show respectively the central estimate and likely range of the time at which 1.5 degrees Celsius is reached if the current rate of warming continues. Figure reproduced with permission from the IPCC report [5].

The need for decarbonising the world's energy system is imperative if we aim to prevent the worst effects of climate change, such as heatwaves and extreme precipitation. Low-carbon energy sources such as solar, wind and hydroelectric power do not contribute to human-induced climate change. Universal access to efficient technologies that make use of renewable energy sources, together with the protection of the environment, is fundamental to secure a habitable planet for future generations.

\subsubsection{The scale of the solar resource}

Of all renewable energy sources, the sun is the most abundant, providing Earth with an average power density of $1000 \mathrm{~W} / \mathrm{m}^{2}$ having considered atmo- 
spheric absorption and scattering [7]. In Reference [8] Richard Perez et al. show a comparison between the finite and renewable planetary energy reserves (in TW-years) estimated in 2015. The authors point out that the solar resource potential is that of the entire planet (excluding oceans), accounting for weather, but assuming perfect conversion efficiency. Furthermore, considering only $4 \%$ of land area, and a conversion efficiency of as little as $20 \%$, solar energy remains the obvious energy solution for the future of the planet.

Solar power (as well as wind power) is intrinsically intermittent, which is a barrier to their integration into the electric grid system due to reliability and stability problems. Solar intermittency is characterized by different types of fluctuations which occur at different time scales. Power fluctuations due to diurnal and seasonal variations are predictable, whereas variations that are related to weather are not [7]. This is a complex problem which is expected to be tackled by a combination of energy transmission and storage solutions, many of which are still under development.

\subsubsection{Advantages of organic photovoltaic devices}

Solar photovoltaic (PV) technologies convert solar radiation directly into electricity, and it is one of the fastest growing energy technologies in the world today [10]. The photoactive materials are conventionally inorganic, such as crystalline silicon (c-Si) wafer-based PV which constitute $90 \%$ of the current global production capacity and are the most mature of all PV technologies [7].

Organic photovoltaic (OPV) cells, which are the materials studied in this thesis, use photoactive layers composed of organic small molecules or polymers. OPVs are promising for a number of reasons, such as an abundance of raw materials, tunability and the possibility of manufacture by printing from solution 
onto large areas of flexible and lightweight substrates in a continuous process at room temperature [11]. Due to their properties, OPV active layers can be used in a wide variety of applications, for example embedded in portable products. Recently, OPV system have also been optimized for indoor light harvesting [12], outperforming silicon when using indoor lighting. The output power and voltage obtained, despite being low, could be sufficient for powering electronic devices and sensors, alleviating the use of batteries. The development of OPV cells have shown a continuous growth in power conversion efficiency (PCE) over time, mainly due to research efforts to produce better performing materials, and due to improvements in device architecture. A chart of the highest confirmed conversion efficiencies for emerging PV research cells, including OPV cells, can be found in Reference [9]. In order for OPVs to succeed in the market, further improved efficiency, stability and cost reduction are required.

\subsection{Outline of this thesis}

This Thesis is structured as follows. The following chapter is an introduction into the theory of organic semiconductors and photovoltaic devices, which is necessary to describe the OPV systems of interest. Chapter 3 describes sample preparation, experimental methods and data analysis approaches used throughout the thesis. Chapters 4, 5 and 6 are the experimental chapters, where different factors that influence the performance of OPV devices are targeted. All experimental chapters include reviews of the relevant literature. Chapter 7 is a summary of the key findings and implications. A more detailed description of sample preparations is included as Appendix A. 


\section{Chapter 2}

\section{The Theory of Organic Photovoltaic}

\section{Devices}

The following is a succinct overview of the properties of organic semiconductors and the working principles of organic photovoltaic devices that will guide and provide context to the photophysical studies in organic photovoltaic systems throughout this thesis.

\subsection{Organic semiconductors}

Organic semiconductors are a range of organic materials (small molecules, oligomers, polymers) that combine the electronic properties of semiconductors with the chemical and mechanical benefits of soft materials. Their main constituents are, as indicated by the word organic, carbon and hydrogen atoms, with a few heteroatoms such as sulfur, oxygen, and nitrogen, and their semiconducting behaviour is intrinsic to the electronic structure consisting of a continuous array of alternating single and double bonds forming a $\pi$-conjugated system $[13,14]$. 
Atomic carbon in its ground state has six electrons, two in a $1 s$ orbital, two in a $2 s$ orbital, and the remaining two in two of the three $2 p_{x}, 2 p_{y}$, and $2 p_{z}$ orbitals. When participating in molecular bonds, these atomic orbitals are linearly combined (mixed) forming hydrid orbitals. There can be mixing between all 4 orbitals, between only three of them or between only 2 of them. When the $2 s$ orbital mixes with two $2 p$ orbitals, three $s p^{2}$ hybrid orbitals are formed, leaving the other $p$ orbital un-hybridised. This is the form taken by carbon in the molecule ethene, $\left(\mathrm{H}_{2} \mathrm{C}=\mathrm{CH}_{2}\right)$, also known as ethylene (Figure $2.1 \mathrm{~A}$ ). The $s p^{2}$ orbitals in one carbon atom form $\sigma$ bonds with the hydrogen atoms and with the other carbon. The electrons in the remaining $2 p_{z}$ orbital of each carbon atom will be paired forming a molecular $\pi$ orbital, with spatial probability density above and below the molecular axis. These molecular orbital may be approximated by a linear combination of atomic orbitals (LCAO) and we show in Figure $2.1 \mathrm{~B}$ a simple energy level diagram illustrating the formation of $\sigma$ and $\pi$ bonds from atomic orbitals in ethylene. The splitting between $\pi$ and $\pi^{*}$ orbitals is lower than the splitting between $\sigma$ and $\sigma^{*}$. In this case, the highest occupied molecular orbital (HOMO) is a $\pi$ orbital and the next higher orbital is the $\pi^{*}$ orbital, which is the lowest unoccupied molecular orbital (LUMO). HOMO and LUMO are also known as frontier orbitals and they play a critical role in the optical and electrical processes of the molecule. Importantly, the weaker splitting between $\pi$ and $\pi^{*}$ results in HOMO-LUMO transitions in the visible spectral range, making these materials suitable for semiconductor applications. 
A

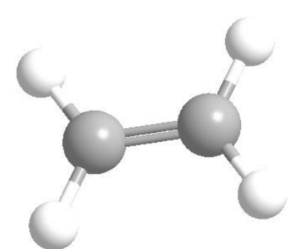

B

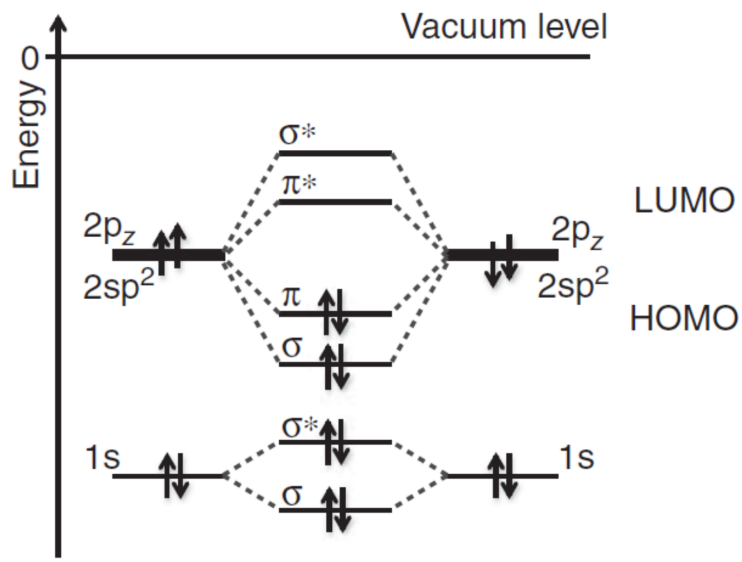

Figure 2.1: A) An illustration of ethylene, the simplest molecule with $s p^{2}$ hybridasation. B) Simple energy level diagram illustrating the formation of $\sigma$ and $\pi$ bonds from atomic orbitals in ethylene. Only orbitals involved in the carbon-carbon interaction are shown. The Figure is reproduced from Reference [13].

The field of research of organic semiconductors originated with the study of molecular crystals, composed by flat, large, aromatic molecules such as the polyacenes, in particular naphthalene, anthracene, tetracene, and pentacene, as well as pyrene, perylene, and similar compounds $[13,14]$. The field was further revolutionised in the 1970s by the discovery that polymers can, after certain modifications, also be made electrically conductive. This is achieved by conjugation along the polymer chains and doping through the addition of suitable atoms. Alan J. Heeger, Alan G. MacDiarmid and Hideki Shirakawa were rewarded with the Nobel Prize in Chemistry in 2000 for the discovery and 
development of conductive polymers $[15,16]$.

\subsection{Electronic and optical processes}

When the organic semiconductor material absorbs a photon, the $\mathrm{HOMO} \rightarrow$ LUMO transition takes place, resulting in the excitation of the molecule. When investigating the excited states, we need to consider the spin of the electrons. The spin of a state is given by the total spin of all electrons in all orbitals. However, since filled orbitals contain paired electrons, those orbitals do not contribute to the total spin and it is sufficient to consider the unpaired electrons of an excited state configuration. In a singlet excited state, the spin of an electron in the $\pi^{*}$ orbital and that of the remaining electron in the $\pi$ orbital are antiparallel, adding up to a total spin of zero. In a triplet state, the spin of the electrons in the $\pi^{*}$ and $\pi$ orbitals are parallel, adding up to a total spin of one. Table 2.1 describes states, characteristic orbitals and characteristic spin configurations of low-lying states of ethylene.

Table 2.1: States, characteristic orbitals and characteristic spin configurations of lowlying states of ethylene $\left(\mathrm{CH}_{2}=\mathrm{CH}_{2}\right)$ [14].

\begin{tabular}{ccc}
\hline State & $\begin{array}{c}\text { Characteristic } \\
\text { orbitals }\end{array}$ & $\begin{array}{c}\text { Characteristic } \\
\text { spin electronic configuration }\end{array}$ \\
\hline$S_{1}$ & $\pi, \pi^{*}$ & $(\pi \uparrow)^{1}\left(\pi^{*} \downarrow\right)^{1}$ \\
\hline$T_{1}$ & $\pi, \pi^{*}$ & $(\pi \uparrow)^{1}\left(\pi^{*} \uparrow\right)^{1}$ \\
\hline$S_{0}$ & $\pi^{2}$ & $(\pi)^{2}\left(\pi^{*}\right)^{0}$ \\
\hline
\end{tabular}


In a diatomic or polyatomic molecule, vibrational and rotational states are superimposed on each electronic state, and when a photon with sufficient energy is absorbed, the molecule is transferred into an excited electronic state, but also acquires vibrational energy [17]. The decay of an excited state in a single molecule is usually represented in a state energy diagram such as Figure 2.2 , which is sometimes referred to as a Jablonski diagram. The $y$-coordinate represents the potential energy of the system and different states are grouped horizontally by spin multiplicity. Due to the conservation of total spin, the lowest optical spin-allowed transition is $S_{0}+h v \rightarrow S_{1}$ which requires a minimum photon energy $(h v)_{\min }$ equivalent to the optical bandgap of the molecule.

In inorganic semiconductors, electrical and optical bandgaps are identical because optical absorption creates a pair of unbound charges. The bandgap is given by the difference between ionization potential and electron affinity. In an organic semiconductor, the optical badgap is different from the electrical bandgap due to the binding energy of the strongly bound excitons that are created upon light absortion. Electron-hole pairs need to overcome their binding energy in order to fully dissociate into an electron and a hole separated by the energy of the electrical gap [18].

When $S_{1}$ or a higher excited state is populated, radiative and nonradiative decay processes take place. The radiative decay known as fluorescence typically occurs from the first excited state to the ground state $S_{1} \rightarrow S_{0}+h v$ with characteristic timescales between $10^{-9}-10^{-6} \mathrm{~s}$. When higher electronic states are populated, the nonradiative decay process to a high vibrational level of a lower electronic state with the same spin, for example $S_{2} \rightarrow S_{1}$, is known as internal conversion. If higher vibrational states for a given electronic state have been populated, decay to lower vibrational states is known as vibrational re- 
laxation (VR) or vibrational cooling, which involves the dissipation of energy from the molecule to its surrounding. The nonradiative decay from $S_{1}$ to $T_{1}$ is a spin-forbidden transition known as intersystem crossing (ISC), where a singlet excitation is converted into a triplet excitation, and its rate is determined by the interaction between the electron's spin motion and its orbital motion, i. e. the spin-orbit coupling [14]. Phosphorescence is also a spin-forbbiden transition $T_{1}+h v \rightarrow S_{0}$ and occurs at very low rates, with characteristic timescales between $10^{-3}-10^{2} s$.

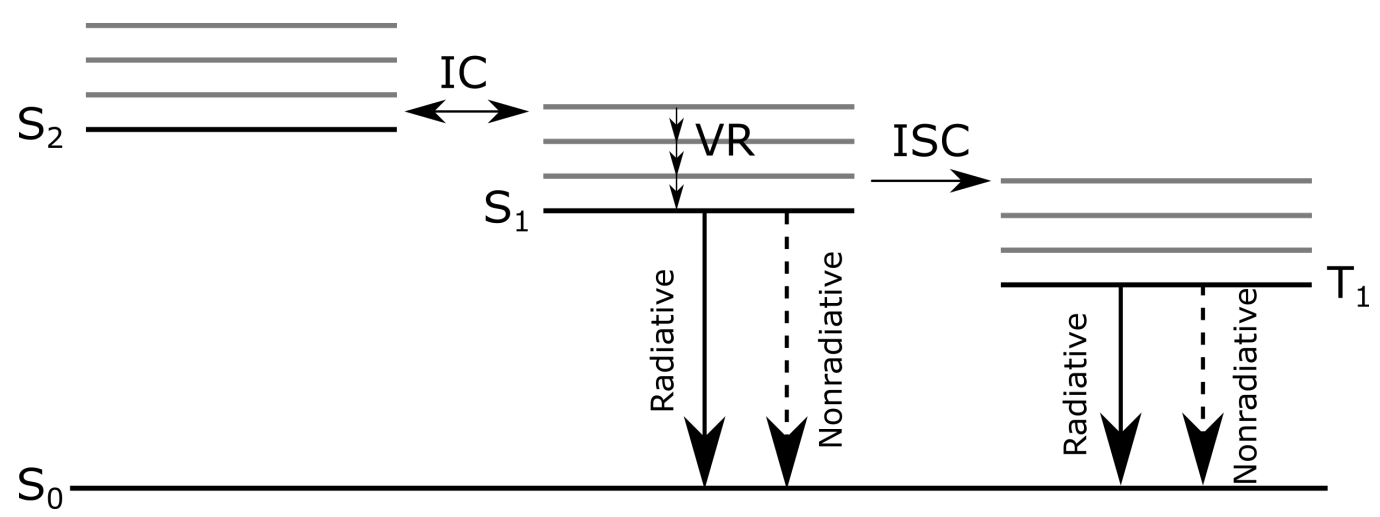

Figure 2.2: Electronic state diagram for a single molecule. $S_{0}$ : singlet ground state, $S_{1}$ and $S_{2}$ : first and second singlet excited states, $T_{1}$ : first triplet excited state, ICS: intersystem crossing, IC: internal conversion, VR: vibrational relaxation. Figure modified from [14].

Optical transitions can be formally described by a quantum mechanical treatment. Electronic transitions are essentially instantaneous, since there is no time for appreciable motion of the nuclei, and the transitions appear as vertical lines between the electronic potential surfaces of the states involved [22]. This is known as the Franck-Condon principle, which is the application of the Born-Oppenheimer approximation to photon absorption and emission. This is illustrated in Figure 2.3, with electronic potential surfaces for the ground 
state and an excited state as a function of the internuclear distance. The FrankCondon principle explains the presence of vibronic structure in the absorption and photoluminescence spectra. However, many organic materials have broad absorption spectra due to the absorption of multiple molecular conformations [23]. Since molecules are bound by weak Van-der-Waals interactions, the electronic structure of individual molecules is only weakly disturbed in the solid phase. Therefore, the optical properties of thin-films and single molecules are quite similar. However, due to the polarization effects, solid state absorption and emission spectra are typically red-shifted compared to the respective molecular spectra [24].

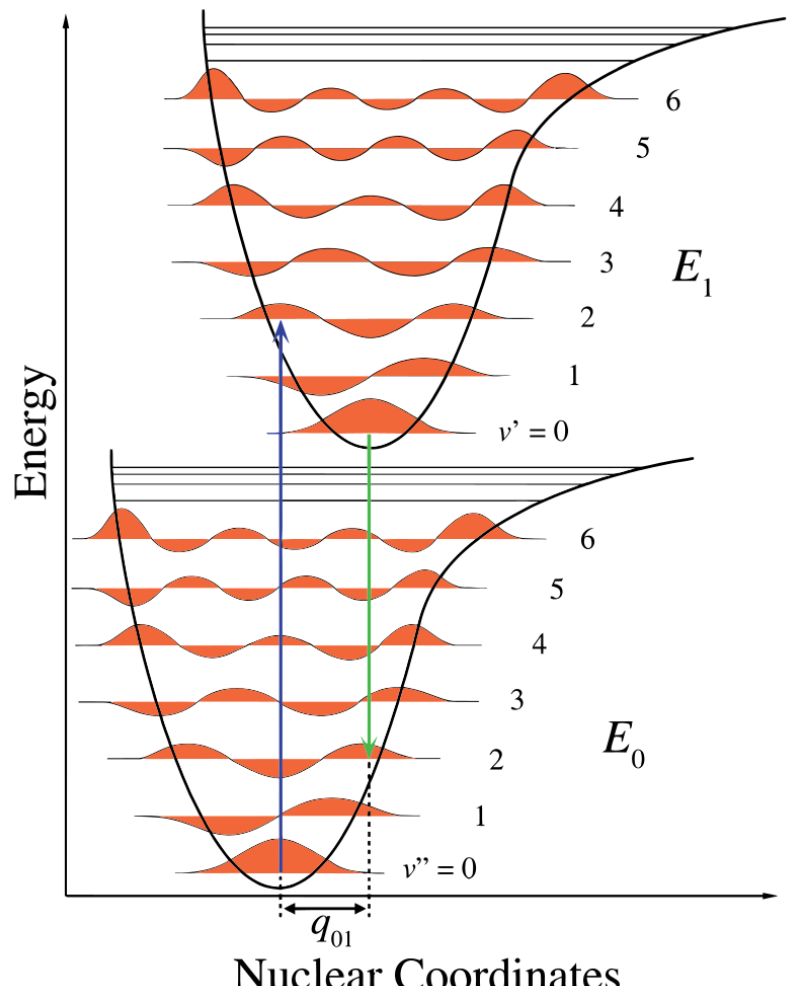

Figure 2.3: Franck-Condon diagram, illustrating the wavefunction overlap between the initial and final vibrational states [25] (Creative Commons license). 
The probability of an optical transition between an initial state $i$ and a final state $f$ can be described in terms of the quantum mechanical approach of perturbation theory. The rate of the optical transition $k_{i f, r a d}$ can be calculated via Fermi's golden rule in the dipole approximation, using the dipole operator $e \hat{\mathbf{r}}$ for the perturbing Hamiltonian [13]:

$$
k_{i f, r a d}=\frac{2 \pi}{\hbar}\left|\left\langle\psi_{f}|e \hat{\mathbf{r}}| \psi_{i}\right\rangle\right|^{2} \rho(E),
$$

where $\psi_{i}$ and $\psi_{f}$ are the wavefunctions of the initial and final states, and $\rho$ is the density of the final states. If the Franck-Condon principle is valid, Equation 2.1 can be written as Equation 2.2, where the dipole operator acts only on the electronic wavefunction.

$$
k_{i f, r a d}=\frac{2 \pi}{\hbar}\left|\left\langle\psi_{e l, f}|e \hat{\mathbf{r}}| \psi_{e l, i}\right\rangle\right|^{2}\left|\left\langle\psi_{\nu i b, f} \mid \psi_{\nu i b, i}\right\rangle\right|^{2} \rho(E) .
$$

According to Equation 2.2, the optical transition rate depends on an electronic factor, which is the transition dipole matrix element $\left\langle\psi_{e l, f}|e \hat{\mathbf{r}}| \psi_{e l, i}\right\rangle$, and a vibrational factor $\left\langle\psi_{\nu i b, f} \mid \psi_{\nu i b, i}\right\rangle$, which is the overlap of the vibrational wavefunctions, also known as the Franck-Condon overlap integral. The square of the Franck-Condon overlap integral is the Franck-Condon factor FC [13].

Nonradiative transitions occur between the zeroth vibrational level of the initial state and a higher vibrational level of the final state. They are horizontal (isoenergetic) transitions in the potential energy curve diagram. The rate of a nonradiative transition $k_{i f, n o n r a d}$ can be calculated by once again invoking Fermi's golden rule, where the perturbing Hamiltonian that induces the radiationless transition is the nuclear kinetic energy operator $\partial / \partial Q$ (See Equation 2.3) $[13]$. 


$$
k_{i f, \text { nonrad }}=\frac{2 \pi}{\hbar}\left|\left\langle\psi_{f}|\partial / \partial Q| \psi_{i}\right\rangle\right|^{2} \rho(E) .
$$

In the case of nonradiative transitions, one has to go beyond the Born-Oppenheimer approximation, as demonstrated by Siebrand [74], by first integrating Equation 2.3 over the electronic coordinates and thereafter inserting the molecular wavefunctions and operators. The result is Equation 2.4, which is a similar expression to Equation 2.2 for radiative transitions, where $J$ is the electronic coupling between the initial and final states and FC is the Franck-Condon factor.

$$
k_{i f, \text { nonrad }}=\frac{2 \pi}{\hbar} \rho J^{2} F C .
$$

Siebrand, Robinson and Frosch $[27,74]$ calculated how the Frack-Condon factor changes with the energy difference $\Delta E$ between the $0-0$ energies of the initial and final state. Considering only the vibrational mode with the highest frequency $\omega_{M}$ of the final state, and evaluating $\mathrm{FC}$ for the case of a displaced and distorted oscillator, FC results in an exponential dependence on $\Delta E / \hbar \omega_{M}$. As $J$ is constant with energy, this leads to an exponential dependence of the nonradiative transition rate on the energy difference between the initial and final states (Equation 2.5), which is known as the energy-gap law.

$$
k_{i f, \text { nonrad }} \propto \exp \left(-B \frac{\Delta E}{\hbar \omega_{M}}\right)
$$

where $B$ is a molecular parameter.

The radiative and nonradiative decay rates are the photophysical parameters that control the lifetime $\tau$ of an excited state, the quantum yield of emission from an excited state, and the quantum yield of transitions between states [13]. The lifetime (the total number of decays per second) of an excited state 
can be calculated from the radiative and nonradiative rates $\left(k_{r a d}\right.$ and $k_{\text {nonrad }}$ respectively) as described in Equation 2.6.

$$
\tau=\frac{1}{k_{\text {rad }}+k_{\text {nonrad }}}
$$

In a TAS experiment, lifetimes can be directly retrieved from the timedependent behavior of populations.

Before focusing on the working principles of organic photovoltaics, we will introduce the Frenkel exciton, which is the elementary excitation in an organic solid, and the process of electron transfer. The dielectric constant $\epsilon_{r}$ in these materials is of around $\epsilon_{r}=3.5$, which is relatively low compared to inorganic semiconductors such as silicon $\left(\epsilon_{r}=12\right)$, germanium $\left(\epsilon_{r}=16\right)$, and GaAs $\left(\epsilon_{r}=\right.$ 13). As a result, upon photo-excitation, a spatially localised electron-hole pair is created, coulombically bound with a relatively high binding energy of $E_{b} \approx$ $0.1-1 \mathrm{eV}$. Such localised electron-hole pair is known as a Frenkel type exciton and does not spontaneously dissociate into free charge carriers due to $E_{b}$ being one order of magnitude greater than the thermal energy $k_{B} T=25 \mathrm{meV}$ at room temperature. Since this is such a distinctive property of organic semiconductors, organic solar cells are also known as excitonic solar cells.

As will be illustrated in the next section, in order to produce useful electrical power, excitons must dissociate into free, extractable charge carriers. Such dissociation is facilitated by a charge transfer process at the interface between an electron-donor material and an electron-acceptor material, i.e. a donor:acceptor heterojunction. A successful analytical description of electron transfer was developed by Marcus in 1956 [28]. Marcus theory has been successfully applied to a number of chemical systems, including the photoinduced charge transfer process in conjugated polymer blends [29]. In Marcus theory, the electronic 
potential surfaces of the ground state, the excited state and the charge transfer state are represented as harmonic oscillators (parabolas), as illustrated in Figure 2.4 .

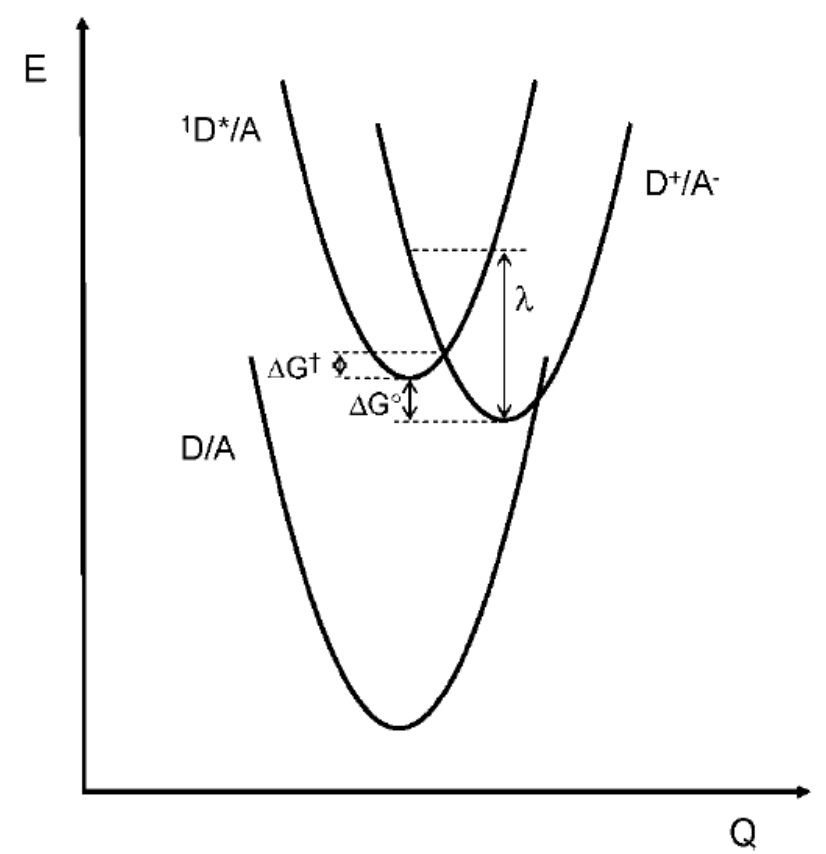

Figure 2.4: Potential energy surfaces for a $D / A$ system, where photoexcitation generates $D^{*} / A$ and subsequent electron transfer generates $D^{+} / A^{-} . \Delta G^{o}$ is the energy difference between the two surfaces' minima, the energy barrier for the reaction is $\Delta G^{\dagger}$, and $\lambda$ is the reorganization energy. Reprinted with permission from [29]. Copyright 2010 American Chemical Society.

According to Marcus theory, electron transfer must occur at the intersection between the parabolas in Figure 2.4, to satisfy both energy conservation and the Franck-Condon principle, which states that the nuclear configuration is 'frozen' during the charge transfer. As a result, the electron transfer is an activated process with an energy activation barrier $\Delta G^{\dagger}$, which according to Marcus is a function of the Gibbs free energy, $\Delta G^{o}$ and the reorganization energy $\lambda$ (see Equation 2.7). 


$$
\Delta G^{\dagger}=\frac{\left(\lambda+\Delta G^{o}\right)^{2}}{4 \lambda}
$$

The reorganization energy $\lambda$ is the energy required to rearrange the initial system such that it matches the final geometry (including changes in the nuclear geometry of the molecules involved in the charge transfer event and of the surrounding medium).

The rate constant for electron transfer $\left(k_{E T}\right)$ is calculated invoking Fermi's golden rule, which results in the following expression,

$$
k_{E T}=\frac{2 \pi}{\hbar} V^{2} F C=\frac{2 \pi}{\hbar \sqrt{4 \pi \lambda k T}} V^{2}\left(-\frac{\left(\lambda+\Delta G^{o}\right)^{2}}{4 \lambda k T}\right)
$$

where $V$ is the electronic coupling between the initial and final states. According to the exponential term in Equation 2.8, which is a Gaussian normal distribution, the rate for electron transfer increases as $-\Delta G^{o}$ increases, until the maximum rate is achieved when $\lambda=-\Delta G^{o}$. A further increase in $-\Delta G^{o}$ results in a decrease in the electron transfer rate, which has been verified experimentally and it is known as the Marcus inverted region [29].

\subsection{The organic photovoltaic device}

The first uses of molecular materials for photovoltaic applications consisted of replacing the typically inorganic photo-active material in a solar cell with an organic material. However, the efficiencies achieved were minimal. The potential of these materials for photovoltaic use became more evident with the use of two materials, an electron donating and an electron withdrawing material (donor and acceptor), in a bilayer architecture [30]. The pioneering work of Tang in 1986, using copper phthalocyanine and a perylene tetracarboxylic 
derivative, already demonstrated that the properties of the interface between the two organic materials, rather than the electrode/organic contacts, is crucial in determining the photovoltaic properties of the device.

The efficiency of organic photovoltaic devices took a leap in the 1990s thanks to two major contributions: 1) the incorporation of fullerene and its derivatives as electron accepting materials, and 2) the invention of the bulk heterojunction device architecture [31-33], where the donor and acceptor materials are blended forming a single interpenetrating active layer. The materials used in these groundbreaking publications are shown in Figure 2.5 and the active layer of a BHJ device is illustrated in Figure 2.6 along with an illustration of a bilayer structure. Fullerenes have an extremely low reorganization energy following electron transfer, a property that makes them one of the most useful electron acceptor molecules in organic electronics. Additionally, their (nearly) spherical structure allows electron transfer to occur in any spatial direction without preference, and their electron mobility is excellent (on the order of $0.1 \mathrm{~cm}^{2} / \mathrm{Vs}$ ) for an organic material [34].

A

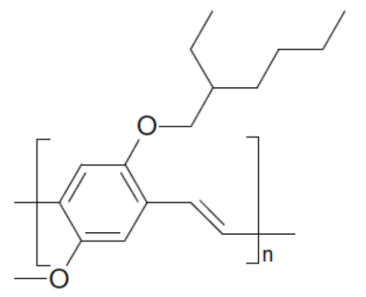

MEH-PPV
B

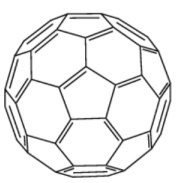

C60
C

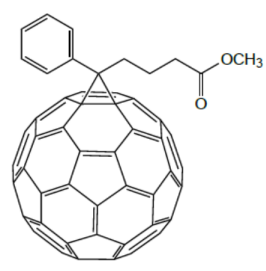

PC61BM

Figure 2.5: Materials composing the active layers of pioneering publications on $\mathrm{BHJ}$ OPV devices in References [31-33]. A) The polymer donor MEH-PPV, B) the electron acceptor $\mathrm{C} 60$, and $\mathrm{C}$ ) the ubiquitous electron acceptor $\mathrm{PC}_{61} \mathrm{BM}$. 
A

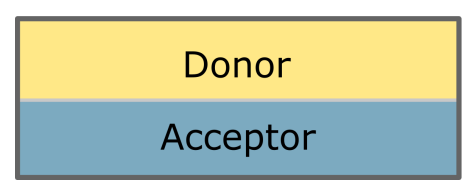

B

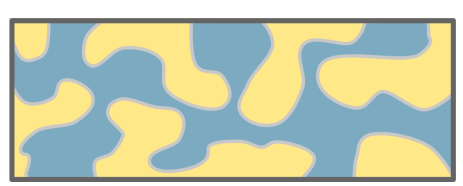

Figure 2.6: Illustrations of A) a bilayer structure, and B) a bulk heterojunction (BHJ) structure in OPV active layer.

For decades, fullerenes were featured in the most efficient OPV systems, however, the efficiency of fullerene devices was reaching a plateau in the past years and today the highest PCEs (featured in Figure ??) have been achieved using nonfullerene small molecules as electron accepting materials. State-of-the-art nonfullerene acceptors can be categorized into two types according to their chemical structure: acceptors based on fused aromatic diimides and acceptors based on strong intramolecular electron push-pull effects, showing great tunability in absorption and electron energy levels. In addition, for solution-processed solar cells, appropriate functional groups also need to be carefully designed to satisfy solubility requirements [35].

One of the requirements for an efficient photovoltaic material is featuring an optimal energy gap for photo-absorption. In Figure 2.7 we show the Air Mass 1.5 (AM1.5) spectrum, which corresponds to the solar irradiance with the sun $45^{\circ}$ above the horizon (Extracted from Reference [36]). The data is given in units of irradiance per bandwidth and units of photon flux, both as a function of wavelength. The latter is particularly useful since photovoltaic devices directly convert photons into pairs of charge carriers (ideally with unitary yield). It is clear from the figure that accessing longer wavelengths is required to harvest a larger number of photons, to achieve high currents and therefore, higher efficiencies. 


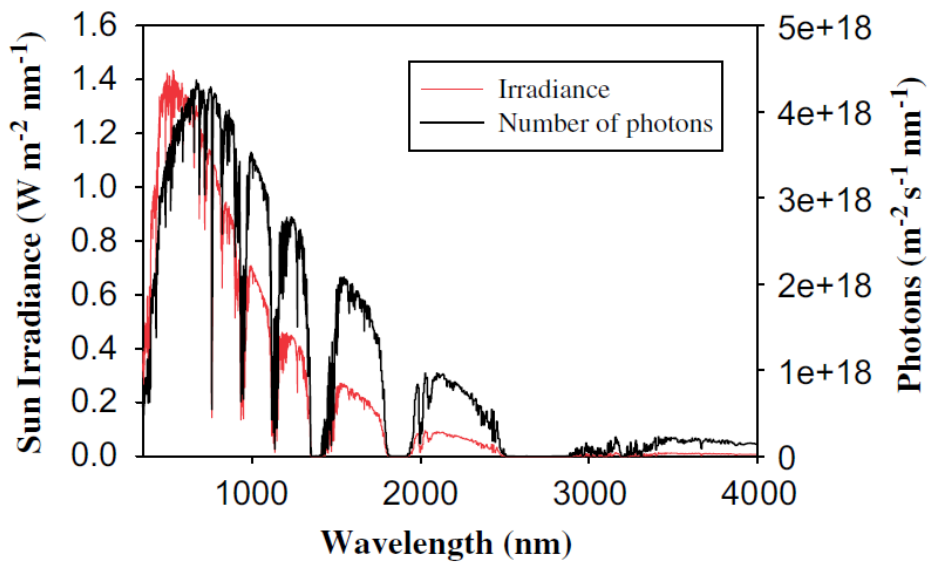

Figure 2.7: Sun irradiance (red) and number of photons (black) as a function of wavelength. The sun intensity spectrum is based on data from the National Renewable Energy Laboratory (NREL). Figure reproduced with permission from Reference [36].

To extend the absorption range of donor polymers, different strategies have been implemented. One strategy consisted of extending the conjugation length of the $\pi$ conjugated segments, for example, by replacing phenyl rings with thiophene rings that help to planarize the polymer backbone through a reduced steric effect [37]. Another strategy involved varying the heteroatoms in the conjugated backbone, e.g. replacing the sulphur atom in polythiophene with selenium, which leads to a lowered LUMO energy but unchanged HOMO [38]. However, the most widely used approach for obtaining low-band-gap polymers is based on the use of alternating electron-rich (donor) and electron-deficient (acceptor) moieties as repeating units in polymer backbones, an approach introduced in the 1990s by Wynberg and co-workers [39]. Due to the combination of high-lying HOMO levels residing on the donor units and low-lying LUMO levels residing on the acceptor units, an overall narrow band gap results [40], and in this approach HOMO and LUMO can be controlled separately [41]. Because of the flexibility of this approach, it became ubiquitous in the synthesis of low- 
band-gap polymers allowing the harvesting of considerably longer wavelengths of the solar spectrum. The term low band gap polymer is used for polymers with a band gap below $2 \mathrm{eV}$, i.e. absorbing light with wavelengths longer than $620 \mathrm{~nm}$.

Some of the polymer donors that are already commercially available today, are shown in Figure 2.8. P3HT is a high-band-gap polymer that has been extensively studied as a donor material in fullerene OPV devices [42]. PffBT4T-2OD, also known as PCE11, is a medium-band-gap polymer that has shown efficiencies above $10.8 \%$ with a wide variety of fullerene-based electron acceptors [43]. PTQ10 is a medium-band-gap polymer that has demonstrated PCEs up to $15.5 \%$ without additive and up to $16.5 \%$ with the additive DIO with the well-known nonfullerene acceptor Y6 [44]. PPDT2FBT is a low-band-gap polymer with long-term thermal stability, which is studied in Chapter 5 of this thesis [45-48]. 


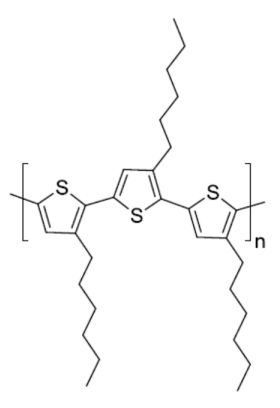

$\mathrm{P} 3 \mathrm{HT}$

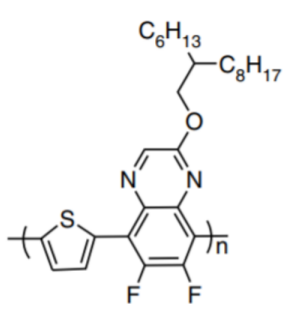

PTQ10

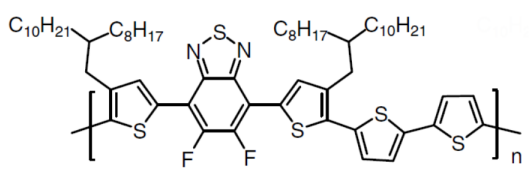

PffBT4T-2OD

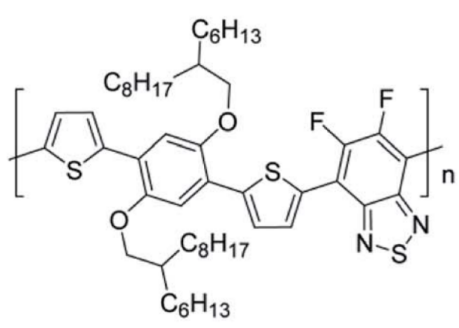

PPDT2FBT

Figure 2.8: Some polymer donors that are commercially available.

\subsubsection{From excitons to extractable charge carriers}

In order to give useful electrical power, dissociation of excitons into free charge carriers must occur in the organic photovoltaic (OPV) device. As mentioned earlier, major breakthroughs were the discovery that dissociation of electron-hole pairs was facilitated at the interface between a donor and an acceptor material, i.e. a donor:acceptor heterojunction, and the introduction of the bulk heterojuction (BHJ) architecture together with the identification of fullerene derivatives as favorable electron acceptors. A vast number of donor:acceptor combinations have been reported to this date, typically featuring conjugated polymers as the electron donors blended with a fullerene derivative as the elec- 
tron acceptor. In summary, the processes involved from exciton formation to charge extraction (illustrated in Figure 2.9) are the following [29, 49, 50]:

1. The exciton reaches the donor-acceptor interface and charge transfer is driven via either electron transfer (ET) or hole transfer (HT), resulting in the population of charge-transfer (CT) states.

2. CT states dissociate into charge carriers, resulting in the appearance of holes (positive polarons) in the donor and electrons (negative polarons) in the acceptor.

3. Charge transport occurs via drift and diffusion in both donor and acceptor phases and, unless they are lost due to recombination, charges are extracted at the electrodes of the device.

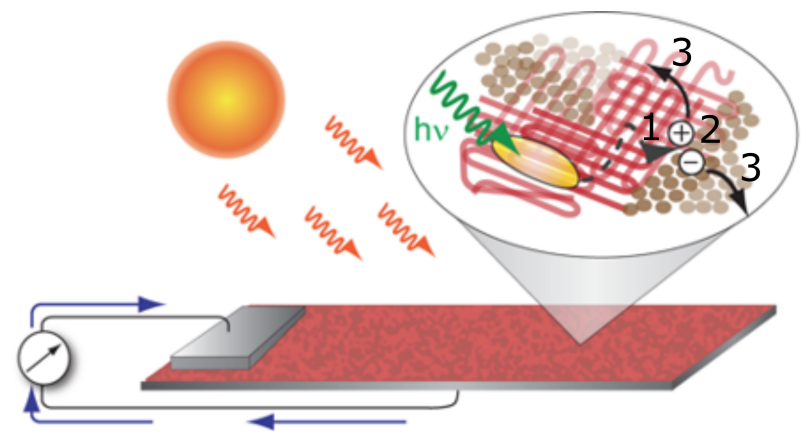

Figure 2.9: An illustration of the processes involved from exciton formation to charge extraction. 1) The exciton reaches the donor-acceptor interface and undergoes charge transfer, 2) CT states dissociate into charge carriers, and 3) Charges are transported through the pure phases and extracted at the electrodes of the device. Figure provided by justin Hodgkiss.

It is important to note that the steps involved in charge photo-generation are not completely efficient, since they compete with different loss processes, such 
as exciton decay, recombination of geminate CT states (known as geminate recombination) and recombination of separated charges (known as nongeminate recombination), which will be discussed in more detail later.

An analytical descripton of geminate recombination was first proposed by Onsager for the dissociation and recombination of ions in solution. Onsager's original theory was refined in 1984 by Braun, who highlighted that the CT state has a finite lifetime [29]. The yield of free carriers was specified by Braun as the product of a dissociation rate $k_{d}(E)$, dependent on the strength of any applied electric field $E$, and the lifetime of the CT states $\tau(E)$, according to Equation 2.9, where $k_{r}$ is the decay rate constant for the CT state to the ground state.

$$
P(E)=\frac{k_{d}(E)}{k_{d}(E)+k_{r}}=k_{d}(E) \tau(E)
$$

The main limitation of the Onsager-Braun model to describe dissociation and recombination processes occuring in bulk-heterojunction OPVs is that the model assumes that carriers exist in a single phase, with equally distributed donor and acceptor molecules. In a bulk-heterojunction, phase separation results in holes living in the donor phase and electrons living in the acceptor phase.

In the OPV community, it is widely accepted that CT states are the species that mediate charge separation and recombination in OPV active layers [51], however, the mechanism by which CT states dissociate at the interface, overcoming the Coulomb attraction, is still not well understood. In the framework of Marcus theory, the difference in energy between the intramolecular singlet excited state on the donor or acceptor and the CT state, provide the driving force for electron-hole separation. Several publications featuring fullerene devices reported a correlation between the frontier energy level offsets and the 
yield of photogenerated free carriers, and an explanation proposed was that the excess energy of the CT state assists in overcoming its binding energy [58]. Bakulin et al. showed experimentally that optical infrared excitation pushes low-energy CT states to higher energies, resulting in a temporary increase in photocurrent [54]. In the same line, Tamura and Burghardt combined electronic structure and quantum dynamical analysis that show that the experimentally observed efficient charge separations can be explained by a combination of two effects. First, the delocalization of charges, which substantially reduces the Coulomb barrier, and second, the vibronically hot nature of the charge-transfer state which promotes charge dissociation beyond the barrier [55]. As a result, it has been postulated that free charges are established via a long-range charge separation within the timescale of CT state thermalization. However, there is no general consensus on the fact that bypassing relaxed low-energy CT states is what leads to charge separation. Transient absorption measurements on high quantum yield OPV systems have shown that electron transfer from a photoexcited polymer, hole transfer from photoexcited fullerene, and prompt electron transfer in intimately mixed polymer-fullerene regions are all equally efficient in generating free carriers, despite the substantial variation of excess energy brought into the system [56-58]. The literature agrees with the role of CT states as mediators in the process of charge generation and recombination. However, whether relaxed or high-energy CT states are the precursors to free carriers is still today under debate. With regard to the driving force, a greater difference between the frontier orbitals at the donor:acceptor interface represents a loss of energy in the charge generation process, as illustrated in Figure 2.10. Recent studies have reported nonfullerene OPV devices where efficient charge separation was achieved despite a negligible driving force [59], demonstrating that high open circuit voltage can be achieved without significantly compromising 
the charge generation efficiency [60].

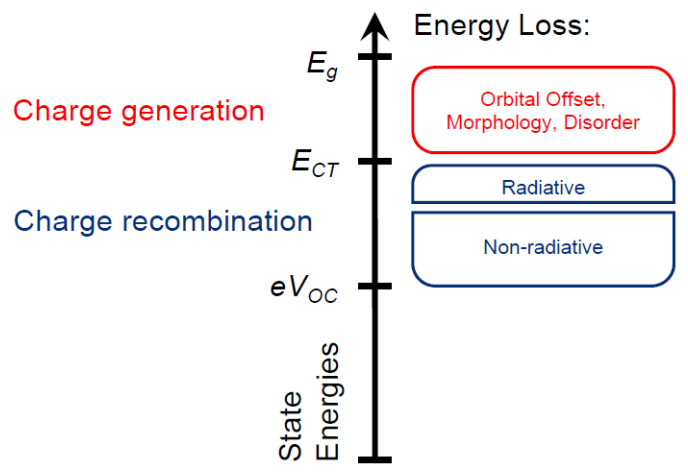

Figure 2.10: Energy loss in an OPV device, separated as losses during charge generation and losses due to charge recombination. Figure reproduced with permission from Reference [52].

A kinetic approach to charge separation and recombination in disordered and annealed P3HT:PCBM blends was carried out by Howard et al. [61]. The authors used transient absorption spectroscopy to study geminate and nongeminate charge recombination and proposed two possible mechanisms for charge generation and recombination, which are illustrated in Figure 2.11. In mechanism 1 the thermal relaxation of the charge-transfer state is much faster than separation and consequently charge separation and recombination are mediated by the relaxed charge-transfer state. An Onsager-Braun type description of charge separation should be applicable in this case. In mechanism 2, the spatial separation of charges in hot charge-transfer states created directly after exciton quenching rivals or exceeds the rate of internal conversion in the CT manifold. In this case, free, spatially separated charges are generated on a very fast time scale. In their study, the authors show that exciton quenching leads directly (in a time scale less than $100 \mathrm{fs}$ ) to two charge populations: bound charges and free charges. They used both kinetic models mentioned before to fit the data accross 
different pump fluences and found that the model that adequately explains the data is the so-called ultrafast charge separation (or two-pool) model (mechanism 2 in Figure 2.11), which consist of a population of bound charges which decay geminately and do not lead to photocurrent in a photovoltaic cell, and a population of charges generated promptly upon exciton quenching, which can be efficiently extracted.

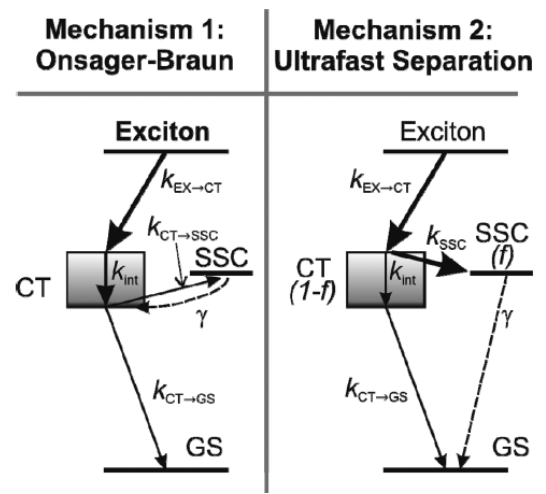

Figure 2.11: Illustrations of two possible mechanisms of charge generation and separation. CT stands for charge-transfer state, SSC for spatially separated (free) charges, and GS for ground state. Reprinted with permission from [61]. Copyright 2010 American Chemical Society.

The ultrafast charge generation model [61] has been used succesfully in a number of studies. According to this model, CT states decay monomolecularly to the ground state, and in parallel, spatially separated charges (SC) also recombine to the ground state following a recombination order of $\lambda+1$. Some of the reported parameters within this framework are shown in Table 2.2, where $k_{C T \rightarrow G S}$ is the CT state recombination rate constant, which has units of $\mathrm{s}^{-1}, \lambda+1$ is the recombination order for separated charges, and $\gamma$ is the recombination rate constant for separated charges, which has units that depend on the parameter $\lambda$ such that the product $\gamma S C^{\lambda+1}$ has units of $d S C / d t$. The rate equations 
Table 2.2: Parameters from global fits reported in the literature using the ultrafast charge separation model in annealed RR-P3HT:PCBM [61], PCPDTBT:PCBM [64] and annealed TQ1:N2200 [62].

\begin{tabular}{cccc}
\hline & $k_{C T \rightarrow G S}\left(\mathrm{~s}^{-1}\right)$ & $\gamma\left(\left(\mathrm{cm}^{3}\right)^{\lambda} \mathrm{s}^{-1}\right)$ & $\lambda+1$ \\
\hline RR-P3HT:PCBM & $2.5 \times 10^{8}$ & $1.9 \times 10^{-20}$ & 2.45 \\
\hline PCPDTBT:PCBM & $1.9 \times 10^{8}$ & $4.0 \times 10^{-10}$ & 1.93 \\
\hline TQ1:N2200 & $4.1 \times 10^{8}$ & $3.2 \times 10^{-18}$ & 2.36 \\
\hline
\end{tabular}

describing this mechanism are given in Equation 2.10.

$$
\left\{\begin{array}{l}
\frac{d C T(t)}{d t}=-k_{C T \rightarrow G S} C T(t) \\
\frac{d S C(t)}{d t}=-\gamma S C(t)^{\lambda+1}
\end{array}\right.
$$

The Onsager-Braun type model, where the CT state mediates charge generation and transport, has also been successful for explaining experimental recombination [62] and [63].

In Reference [66] the authors review models based in the Onsager-Braun theory and several theoretical efforts that have been focused on understanding the energy and nature of the electronic states at the donor-acceptor interface. Figure 2.12, extracted from Reference [66], illustrates different proposed mechanisms for efficient charge generation in OPVs, including intramolecular or intermolecular delocalisation of charge, which may result in a higher effective electron-hole separation, and reduced binding energy in the CT state, disorder at the interface, which may drive charges into single component domains, the built in electric field and the delocalisation of the initial excitation, with formation of separated charges without the intermediary interfacial CT state. 
(a)

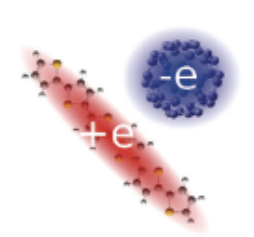

(C)点政

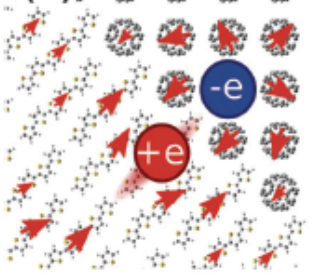

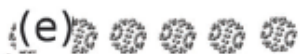

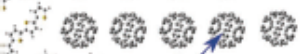
से

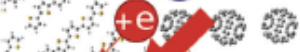

स $\mathrm{F}$. से से के

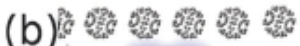
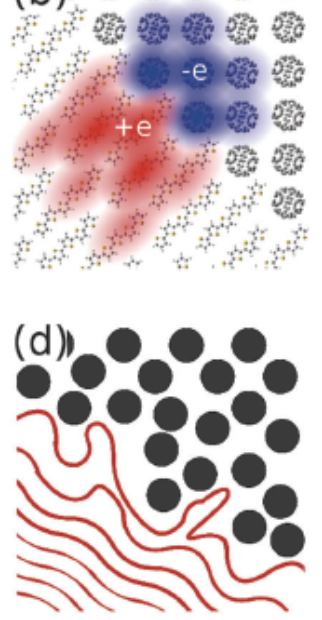

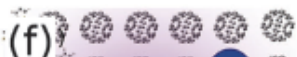

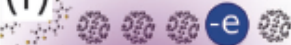

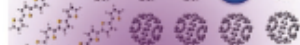
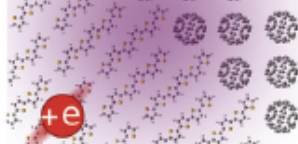

Figure 2.12: Schematics of proposed mechanisms for efficient charge generation in OPVs. a) Intramolecular or b) intermolecular delocalisation of charge may result in a higher effective electron-hole separation, and reduced binding energy in the CT state, c) a nonlinear molecular scale treatment of dielectric effects of the surrounding medium may result in changes to the energetic landscape for charge separation, d) disorder at the interface may drive charges into single component domains, e) the built in electric field may drive electrons and holes apart at the interface, and f) the initial excitation may be well delocalised, and directly form well separated charges without proceeding via an interfacial CT state. Figure extracted from Reference [66] (Creative Commons license). 


\subsubsection{Photovoltaic parameters}

OPV devices typically consist of layers of glass (or plastic)/indium tin oxide (ITO)/polyethylenedioxythiophene:polystyrene sulphonate (PEDOT:PSS)/photoactive blend layer/cathode interlayer (such as $\mathrm{Ca}$ or $\mathrm{LiF} / \mathrm{Al}$ ). A similar structure with inverted polarity consists of layers of glass (or plastic)/ITO/metal oxide/photoactive blend layer/PEDOT:PSS/Ag, which is widely studied, mainly because no evaporated metal layers are required [41]. Both device structures are shown in Figure 2.13.

(a)

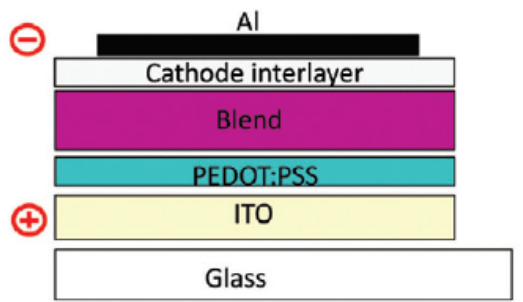

(b)

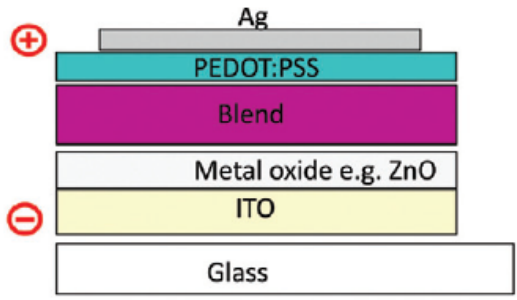

Figure 2.13: Layer structure of an organic photovoltaic device in the traditional a) and inverted b) polarity. Figure extracted from Reference [41] (Creative Commons license).

Under illumination, such device can take the place of a battery in an electric circuit, developing a voltage $V$ between zero and the open-circuit voltage $V_{o c}$ and delivering a current $I$ such that $V=I R_{L}$ where $R_{L}$ is the load resistance. The most fundamental characteristic of a photovoltaic cell is the power conversion efficiency $P C E$ which is defined as the ratio between the output electric power density $P_{\text {out }}=V J$ and the input solar power density $P_{i n}$. It can be calculated from a current density-voltage J-V measurement (Figure 2.14) under standard test conditions: air mass 1.5 spectrum and an incident irradiation of $P_{i n}=$ $100 \mathrm{~mW} / \mathrm{cm}^{-2}$ at $25^{\circ} \mathrm{C}$ [67]. 


$$
P C E=\frac{V J}{P_{\text {in }}}
$$

The fill factor $F F$ is the ratio of the maximun theoretical power (at MPP, the maximum power point) to the product of the short-circuit current and the open-circuit voltage. It is equivalent to the ratio between the light-blue and the blue areas in Figure 2.14 and is a measure of the "squareness" of the $J-V$ curve. By introducing the fill factor $F F$, the power conversion efficiency can also be expressed by equation 2.12, where $V_{o c}$ is the open circuit voltage and $J_{s c}$ is the short-circuit current density.

$$
P C E=\frac{V_{o c} J_{s c} F F}{P_{i n}}
$$

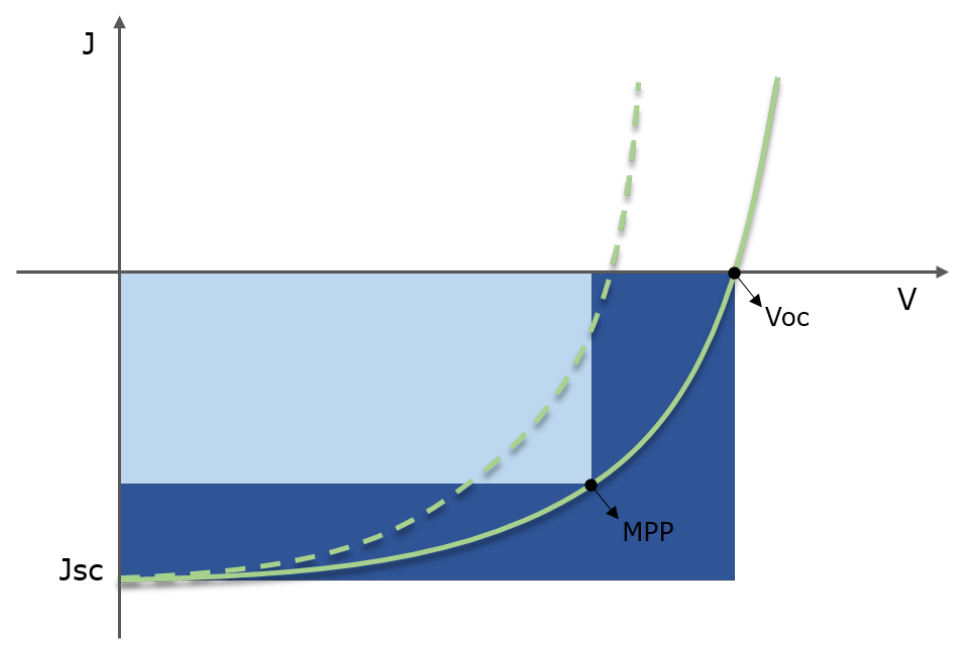

Figure 2.14: A typical current-voltage J-V curve. $J_{s c}$ is the short-circuit current density and $V_{o c}$ the open-circuit voltage. MPP is the maximum power point.

The open-circuit voltage $V_{o c}$ is the maximum voltage generated by the device and it was thought to be mainly determined by the energy difference between 
the energy levels of the LUMO of the acceptor and HOMO of the donor. However, it was demonstrated in recent years that $V_{o c}$ relates to the molecular properties at the donor:acceptor interface, i.e. the energy of the interfacial CT excitons rather than the HOMO-LUMO gap [68]. The short-circuit current $J_{s c}$ is the current density measured when the applied voltage is zero and it is the maximum current extractable from the device. $J_{s c}$ is strongly determined by the optical gap of the photo-absorbing material. The correlation between the fill factor and the material properties has been evaluated by Neher et al., and the authors introduced a new figure of merit $\alpha$, which relates characteristic parameters like the fill factor or the apparent series resistance of the device to the charge carrier mobilities, the active layer thickness and the bimolecular recombination coefficient [69]. Improvements in device efficiency are achieved when one or more of these figures of merit are enhanced simultaneously and therefore it is crucial to understand what are the molecular factors influencing these parameters.

\subsection{Recombination losses}

\subsubsection{Exciton decay}

Excitons are the precursors for charge photogeneration, and they need to diffuse to the donor-acceptor interface in order to dissociate. Therefore, the thickness of pure donor and pure acceptor domains in a $\mathrm{BHJ}$ must remain limited by the exciton diffusion lengths $[23,70,71]$. If instead, excitons are created at longer distances from the heterojunction interface, exciton decay takes place. A clear overview of singlet exciton diffusion and lifetimes can be found in Reference [72]. Exciton diffusion can be described as a series of dipoledipole induced nonradiative energy transfer steps between the neighboring 
chromophores, described by the Forster Resonance Energy Transfer (FRET) mechanism. The figure of merit for exciton diffusion is the exciton diffusion length $L_{D}$, which depends upon the diffusion constant $D$ and the exciton lifetime $\tau$. The exciton lifetime is determined by radiative and nonradiative decay to the ground state by Equation 2.6, with the nonradiative decay being predominant in the case of large hydrocarbon molecules. In Reference [72] the authors showed that, for a set of fourteen conjugated polymers, the energy-gap law (Equation 2.5) may be valid for the rate of singlet exciton decay.

The improvement of PCE through the use of photoabsorbing materials with large diffusion lengths is currently an active area in the community and remarkably rapid exciton diffusion has recently been demonstrated in a new class of nonfullerene acceptors called fused-ring electron acceptors [75].

\subsubsection{Geminate recombination}

The charge transfer process at the donor:acceptor interface results in a hole in the donor and an electron in the acceptor, which continue to experience strong Coulombic forces. Such electron-hole pair at the interface is known as a polaron pair, geminate pair or charge-transfer (CT) state, which is coupled to the ground state and decays in a monomolecular process that scales linearly with density, and therefore with illumination intensity. The kinetic profile of CT states decaying via gemiante recombination can be described by a single exponential decay, as Equation 2.13,

$$
C T(t)=N_{0} \exp \left(-k_{r} t\right)
$$

where $N_{0}$ is the initial charge carrier density and $k_{r}$ is the geminate recombination constant. Contributions to $k_{r}$ include both radiative and nonradiative 
processes, and the CT lifetime can be calculated using Equation 2.6. As noted earlier, in organic systems nonradiative losses are predominant.

Although the magnitude of the recombination constant is of interest, the ultimate goal is to be able to link these value with molecular properties. The work of Nguyen et al. in bilayer devices in Reference [76] addresses the impact of interfacial molecular orientation on radiative recombination and charge generation efficiency and the authors demonstrate that when the donor/acceptor interface is edge-on (where the $\pi$-faces of the donor and acceptor $\pi$-conjugated molecules or polymer chains are orthogonal), charge generation is more efficient, as evidenced by a higher IQE. This is attributed to reduced CT state-GS electronic coupling as well as smaller activation energy for charge generation in the edge-on bilayer, which may be a consequence of a reduced barrier between CT state and charge-separated states or favorable polarization at the donor/acceptor interface. Interestingly, high performance nonfullerene OPV devices recently reported have shown minimal geminate recombination $[60,77]$.

\subsubsection{Nongeminate recombination}

Dissociation of CT states at the interface results in the population of separate charge carriers in the donor and acceptor phases. Ideally, charge carriers diffuse to the electrodes where they are drawn to produce useful electricity in an external circuit. However, due to the large interfacial area in a $\mathrm{BHJ}$ device, charges may encounter each other and cancel out, a process refered to as nongeminate recombination. This type of loss is enhanced by the low electron and hole mobilities in organic materials. Groves and Greenham have explained in Reference [78] that the total rate of nongeminate recombination can be considered as being due to two sequential processes. First, the rate at which a pair of carriers within 
a random ensemble are transported toward one another. And second, the efficiency with which a pair of carriers in close proximity recombine, which is determined by the competition of the hopping rate with the recombination rate for adjacent carriers, reminiscent of the Onsager-Braun model discussed earlier.

Transport controlled recombination of free carriers is generally described as a bimolecular process, since it involves the encounter of two species. Such a recombination process is analogous to the recombination of ions in solution, which can be described by the Langevin theory, where the recombination rate $\gamma$ depends on the sum of the mobilities of the two species involved and the recombination process is bimolecular. The Langevin expression for $\gamma$ is presented in Equation 2.14,

$$
\gamma=\frac{q\left(\mu_{e}+\mu_{h}\right)}{\epsilon_{0} \epsilon}
$$

where $q$ is the electronic charge, $\mu_{e}$ and $\mu_{h}$ are the electron and hole mobilities, $\epsilon_{0}$ is the permittivity of free space, and $\epsilon$ is the relative permittivity. When spatially separated charges (SC) decay to the ground state in a bimolecular manner, the population of SC as a function of time can be described analytically by Equation 2.15,

$$
S C(t)=\left(\lambda \gamma t+N_{0}^{-1}\right)^{-1 / \lambda}
$$

where $\lambda+1$ is recombination order, $\gamma$ is the bimolecular recombination constant, also known as the Langevin constant, and $N_{0}$ is the initial charge carrier density.

Experimentally, it has been found that in most OPV systems Langevin theory fails to explain nongeminate recombination and recombination dynamics depends upon mobility, temperature, electric field, charge carrier concentration, 
and trapped charges [52]. One of the limitations of the Langevin bimolecular recombination constant described by Equation 2.14 was addressed by Koster et al. in Reference [79], where the authors explained that, as a consequence of the confinement of both types of carriers to the donor and acceptor phases, the fastest charge carrier reaches the interface first and the time taken by the slowest charge carrier to reach the donor:acceptor interface is the recombination ratelimiting step. The authors could explain experimental J-V curves successfully via drift-diffusion simulations when Equation 2.14 was replaced by Equation 2.16.

$$
\gamma=\frac{q \min \left(\mu_{e}, \mu_{h}\right)}{\epsilon_{0} \epsilon}
$$

Recombination orders in bulk-heterojunction OPV devices have also been found to disagree with Langevin theory, since experimental orders are usually higher than two. Recombination orders of three have been reported, which could suggest an Auger recombination process, where an electron and a hole recombine giving energy to another free electron, which is excited into a higher state. Ultimately that energy is lost as the excited carrier relaxes to the band edge. The rate of Auger recombination is proportional to the densities of the three carriers and therefore the process is only important at very high carrier densities, usually not relevant for OPVs under 1 sun operating conditions. Recombination orders close to 3 are commonly found in OPVs, however they have been explained by introducing disorder in the models via the incorporation of tails in the DoS.

It is worth reminding the reader that in TA experiments, pump fluences are much higher than in solar conditions, in order to achieve signal-to-noise ratios high enough to be able to measure a signal. In such conditions the process of exciton-charge annihilation (ECA) is indeed relevant when the densities of 
excitons or charges are very high. The process of ECA depends on the exciton oscillator strength. The recombination order that have been measured in OPV systems can not be explained via an ECA process, since CT states exhibit very low electrolumninescence due to their typically low oscillator strength.

Different explanations for higher-order recombination include the trapping of charges in the tail states of the density of states (DOS) and mobilities being charge carrier density dependent. In Reference [80] the authors demonstrated, in a system where they deliberately introduced TCNQ electron traps in blends of MDMO-PPV:PCBM, that the open circuit voltage and its light-intensity dependence are strongly affected, not only by bimolecular recombination, but additionally by recombination of holes with trapped electrons, a process also known as Shockley-Read-Hall Recombination. The authors show that the main indication of trap-assisted recombination is the enhanced dependence of the open circuit voltage on the light intensity.

Since opposite free charge carrier need to encounter at the donor:acceptor interface in order to recombine, the morphology of the OPV active layer has a large effect on the nongeminate recombination process. In Reference [81] the authors carried out time delayed collection field (TDCF) measurements to determine the nature of voltage dependent recombination losses in polymer:fullerene devices as a function of processing conditions. The researches demonstrated that annealing devices partially suppresses both geminate and nongeminate recombination, thereby increasing the $F F$ and $J_{s c}$. Additionally, processing devices with an additive allows free carriers to be generated independent of the internal field at the same time as reducing bimolecular recombination. 


\subsection{Research questions addressed in this thesis}

To produce better performing OPV materials in a rational way, it is necessary to understand the relationships between material properties (e.g. energy levels, recombination rates, charge carrier mobilities) and the photovoltaic parameters. And to construct universal relationships, it is essential to improve our understanding of the mechanisms of charge photo-current generation. Significant progress has been made along the years, reflected by the continuous improvement in device performance, however, fundamental aspects of the photo-physics of these systems are still under debate. One of such questions is whether relaxed or high-energy CT states are the precursors to extractable charge carriers. In terms of kinetic models, the ultrafast charge separation (or two-pool) model has been the most widely used to explain fluence-dependent recombination of charges, and such model is associated with the generation of charges via high-energy CT states that are dissociated in an ultrafast time scale.

In this thesis, we use transient absorption spectroscopy (TAS) to directly measure the fate of the photo-excited species created upon light absorption and we investigate charge generation, dissociation and recombination in OPV systems of interest, with two questions in mind. First, we aim to identify the factors limiting the performance of each system. Second, for systems with fluence-dependent charge decay, we test whether the data can be explained with a kinetic model where CT states mediate charge generation and recombination. We therefore use a model reminiscent of the Onsager-Braun model, composed of vibrationally cold CT states which dissociate into SCs and SCs recombining nongeminately to the CT state, with all recombination to the ground state occurring via cold CT states at the donor:acceptor interfaces. Questions related to each particular system under study are additionally addressed. 
In Chapter 4 we explore a family of related polymers based on the low-bandgap alternating copolymer PTBT, where the sulphur atom in the thiadiazole unit was substituted with oxygen or selenium in order to investigate how atom substitution affects the photophysics in this family of related polymers, and in particular the effect of the heavy atom selenium.

Chapter 5 comprises the spectroscopic comparison between fullerene and nonfullerene (NFA) OPV layers featuring the low-band-gap copolymer PPDT2FBT. A specific goal in this chapter is to explain the unexpected low value of $J_{s c}$ in the device featuring a small molecular acceptor, despite of its broad absorption due to complementary absorption of donor and aceptor.

Finally, in Chapter 6 we investigate charge carrier dynamics in a series of low-donor-content films composed predominantly of C60 and small amounts of organic small molecule donors, where their CT state energies are systematically varied. We aim to model charge recombination dynamics to understand how recombination is affected by a molecular factor such as the energy of the CT state. 


\section{Chapter 3}

\section{Experimental Methods and Data}

\section{Analysis}

\subsection{Sample preparation}

The OPV active layers studied in Chapters 4 and 5 of this thesis consist on blend films fabricated in our laboratory using materials developed by our collaborators from Korea University in the research group of Prof. H. Y. Woo. The general fabrication procedure consisted of blending donor and acceptor materials in a solvent, at a particular donor:acceptor ratio (w/w) and total concentration of donor+acceptor. In some cases a volume fraction of an additive was used. Solutions were usually stirred overnight before being spin-coated and post thermal annealing was performed in some cases. It is important to note that the incorporation of additives and thermal annealing have strong effects on the morphology. Solvent additives can affect polymer conformations and thermal annealing alters the film microstructure after casting by allowing the polymer chains more time to equilibrate [82]. 
The samples studied in Chapter 6 were fabricated by our collaborators from University of Postdam in the research group of Prof. Dieter Neher.

\subsection{UV-Vis absorption spectroscopy}

The light absorption properties of chromophores in a material are measured experimentally using steady-state UV-Vis spectroscopy. The spectrometer consists of white light from a tungsten lamp, which is dispersed by a monochromator in combination with a slit. The monochromatic beam is then split into two arms, a reference beam which passes through a blank sample (containing pure solvent in the case of a solution or a blank substrate in the case of a film), and a beam which passes through the sample under study. The intensities of the transmitted beams are recorded for the blank $\left(I_{0}\right)$ and for the sample $(I)$ at each wavelength to compute the absorbance $A=\log \left(\frac{I_{0}}{I}\right)$ of the sample. The absorbance depends on the pathlength and the concentration of the species in the sample according to the Beer-Lambert law presented in Equation 3.1.

$$
A=\log \left(\frac{I_{0}}{I}\right)=\epsilon(v) c d
$$

where $\epsilon(v)$ is the molar attenuation coefficient of that material, $c$ is the molar concentration and $d$ is the pathlength.

In TAS studies, it is a standard practice to measure the ground UV-Vis absorption spectra of samples to determine the required wavelength for the excitation pulses in the TAS experiment. Additionally, we measure the UV-Vis absorption after the TAS experiment as a way to verify that no degradation has occurred during the experiment. Steady-state absorption spectra can also reveal aggregation properties in the material [83]. 


\subsection{Transient absorption spectroscopy}

The development and characterization of intense and ultra-short laser pulses is currently very advanced and laser pulses are the best suited tools for the investigation of dynamic processes in the field of light-matter interaction. The basic principle of operation of a laser (Light Amplification by Stimulated Emission of Radiation) is the process of stimulated emission, where an initial photon serves as a stimulus for excited atoms to decay emitting an identical photon. The nature of this emission in addition to the characteristics of the amplification determine unique properties of laser light, such as high intensity, coherence, directionality and monochromaticity $[84,85]$.

Transient absorption (TA) spectroscopy is the optical pump-probe technique where we use a monochromatic laser pulse to pump or excite a sample and a broadband (white light) laser pulse to probe the dynamics of the photo-excited states, by monitoring their transmission. The ability to directly measure the temporal evolution of excited states, in particular of non-radiative states, makes this technique well suited for the study of charge generation and recombination in organic solar cells. Figure 3.1 illustrates a simplified version of our TA system in the most common configuration. 


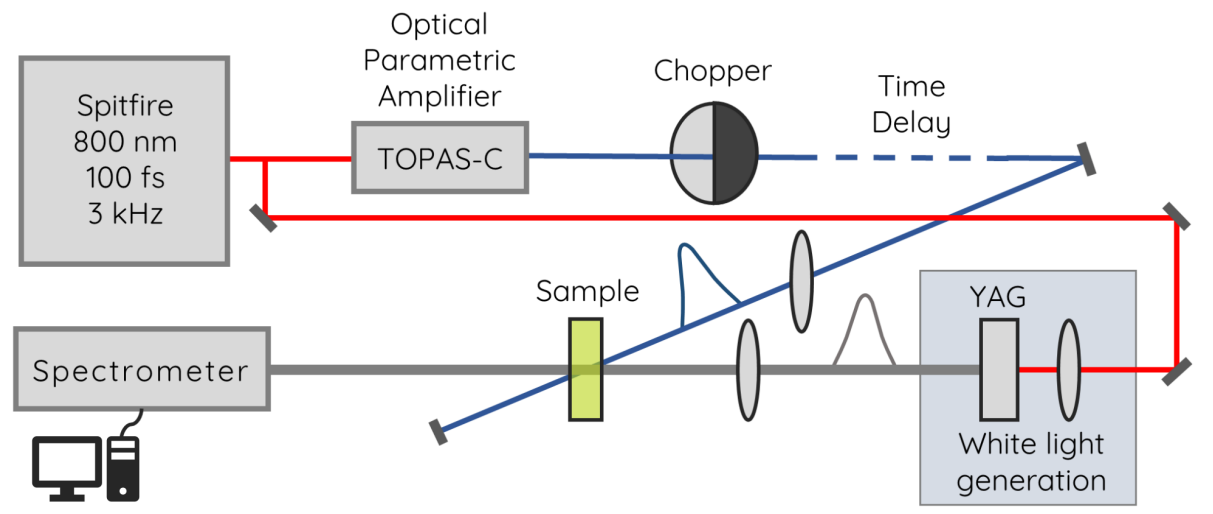

Figure 3.1: A simplified version of our transient absorption spectrometer in the most common configuration, which allows tuning of the pump pulse energy using a parametric amplifier. Pump-probe time delays, obtained by delaying the pump with respect to the probe by increasing its path length, are limited to $6 \mathrm{~ns}$.

Our laser system is based on a Ti-Sapphire regenerative amplifier (SpitfirePro, SpectraPhysics) which provides $800 \mathrm{~nm}$ fundamental $100 \mathrm{fs}$ laser pulses. The standard configuration of our setup consists on the splitting of the fundamental beam into two arms, one for the pump and one for the probe. The temporal resolution of the system is achieved by delaying the pump with respect to the probe by increasing its path length, using a retroreflector mounted in a mechanical delay stage $[86,87]$. In such configuration, we access pump-probe time delays up to $6 \mathrm{~ns}$.

To extend out time delay span up to the microseconds, we use a picosecond laser as the pump source which provides pulses characterised by $1064 \mathrm{~nm}$ wavelegth and $0.6 \mathrm{~ns}$ duration (FWHM), which can be electronically delayed with respect to the probe arm resulting in time delays up to $3 \mu$ s. This is achieved using a signal "T" from the laser amplifier (Spitfire) as the external trigger of a delay generator. The delay generator is controlled via a LabVIEW software 
which determines the different time delays. An output signal "T+delay" from the delay generator is then used as the trigger for the picosecond laser. Additional excitation wavelengths available in this configuration are the second harmonic $532 \mathrm{~nm}$ and third harmonic $355 \mathrm{~nm}$ of the $1064 \mathrm{~nm}$ source.

The repetition rate of our probe beam is $3 \mathrm{kHz}$ and by chopping the pump arm at every second pulse we are able to avoid the use of a reference channel. Rigorously, the probe ground state transmission should be measured via a reference channel, splitting the probe beam before reaching the sample. However, because of the high correlation between sequential pulses, it can be assumed that the ground state signal that corresponds to shot number $i$ is the same as for shot number $i+1$ [88]. In this way, transmitted probe pulses through the excited and the ground sample ( $T_{\text {on }}$ and $T_{\text {off }}$ respectively) are recorded at each time delay $t$. Probe pulses are dispersed with silica prisms and the dispersed light is focused into a visible and infra-red cameras. As a result, the TA signal is calculated as the probe transmission change normalised by the transmission of the ground state $\left(\frac{\Delta T}{T}\right)$ according to equation 3.2.

$$
\frac{\Delta T}{T}=\frac{T_{\mathrm{on}}(\lambda, t)-T_{\mathrm{off}}(\lambda)}{T_{\mathrm{off}}(\lambda)}
$$

In each experiment we excite the sample at specific electronic transitions and to achieve that we tune our pump pulses to the required wavelegth using a parametric amplifier (TOPAS). As explained earlier, when using the picosecond laser as the pump source, only specific wavelengths are available. The broadband probe is generated by focusing one of the $800 \mathrm{~nm}$ arms onto a $3 \mathrm{~mm}$ YAG crystal.

Before each TA measurement, the pump area was recorded at 100 different time delays using a Microsoft webcam and custom LabVIEW software. The 
pixel size of the camera was known, and images of the spot size were recorded for each measurement. The images were subsequently processed performing a gaussian fit in both dimensions, resulting in FWHMx and FWHMy average values. Once the pump area was obtained, the pump fluence $f$ was calculated as the ratio of the pump power $P_{\text {pump }}$ (measured with a Thorlabs power meter which delivers an optical power that depends on the wavelength of the incident beam) and the product of the beam area $A_{\text {pump }}$ times the pump repetition frequency $\left(R_{\text {pump }}=1500 \mathrm{~Hz}\right)$, as described in Equation 3.3.

$$
f=\frac{P_{\text {pump }}}{A_{\text {pump }} R_{\text {pump }}}
$$

Each TA experiment results in a data matrix $M$ where each row is the $\frac{\Delta T}{T}$ spectra at a particular time delay. Subsequently, we subject each data set to a series of corrections:

- Background subtraction, to avoid time-independent artifacts by subtracting the spectra at negative time from the data at positive times.

- Chirp correction. When a broadband laser pulse travels through a dispersive media, the different frequency components travel at a different velocity (longer wavelengths exceeding shorter wavelengths) and therefore time zero (the time at which pump and probe overlap temporaly) is wavelength dependent. By correcting the data due to chirp, we obtain the transient spectrum at each delay time.

- Wavelength calibration, to map camera pixels to wavelengths. We achieve this by calibrating our prism spectrometer using filters ranging $450-$ $1600 \mathrm{~nm}$. 
After fine tuning the prism spectrometer and right before running a TA measurement, we record the spectra of the probe after passing through a set of different filters, which allows us to correlate pixels to wavelengths. The post processing software performs the wavelength calibration taking into account the nonlinear angular dispersion equation, which relates the prism output angle with the incident angle, the apex angle and the index of refraction [88].

The amount of unavoidable chirp due to the WL generation process inside the YAG crystal and the probe transmission through the sample under study is corrected prior to any data analysis. For chirp correction, a time offset is determined as a function of wavelength via fitting a third order polynomial to the wavelength dependent onset of the TA signal [88].

For the interpretation of TA spectra we acknowledge that TA spectra contains contributions from all the different absorbing excited states, and that each component (or species) contributes with different TA features. Such features (illustrated in Figure 3.2) are:

- Ground state bleach (GSB). The excitation of the sample creates a depopulation of the ground state and as a result, spectral regions with ground state absorption exhibit a decrease in transmission of the probe. This feature is known as ground state bleach and according to our convention (Equation 3.2) it results in a positive TA signal.

- Stimulated emission (SE). When probe photons pass through the excited sample with energy that is resonant with a decay transition, they will stimulate the decay where an identical photon is emitted. This process, known as stimulated emission, also results in a positive TA signal.

- Photoinduced absorption (PIA). When the probe is resonant with the 
absorption of an excited species, the probe transmission is reduced and, according to our convension (Equation 3.2), this results in a negative TA signal.

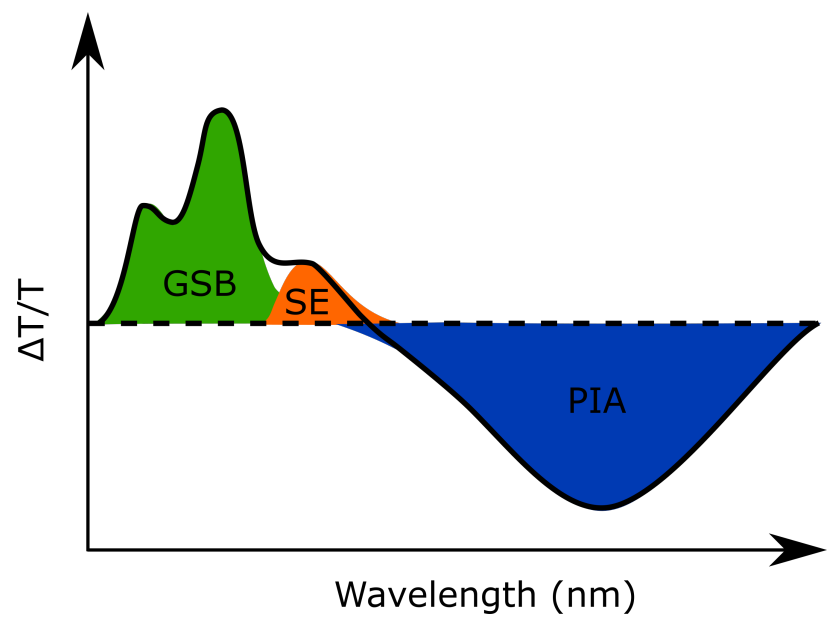

Figure 3.2: Constructed TA spectrum exhibiting features of ground-state bleach (GSB, green), stimulated emission (SE, orange) and photo-induced absorption (PIA, blue). Figure adapted from Reference [89].

In Figure 3.3 A we present a typical TA surface, where the intensity of the TA signal is plotted against the energy of the probe pulse on the $y$ axis and the pump-probe time delays in the $x$ axis. Our analysis of the data begins with the visualization of orthogonal slices of this surface. First, we obtain a series of slices of the TA matrix at different time delays, as shown in Figure $3.3 \mathrm{~B}$, which allows us to inspect the shape of the spectrum, identifying different TA features, and verifying if the spectrum exhibits any temporal evolution, i.e. if the shape of the TA features changes over time. In our example we can identify a positive GSB feature, a negative PIA feature and an additional PIA-2 feature. Having identified these features, we proceed to obtain slices of the matrix over each of the spectral regions, to inspect their temporal profiles. In Figure 3.3 C we show 
kinetic profiles that have been obtained by direct integration over the energy regions associated with each TA feature. The combination of spectral slices and integrated kinetic profiles allows us to carry out the spectral assignments on the TA matrix, i.e. the identification of the transient species contributing to the data. However, the spectral assignment usually requires additional information, such as known spectra for triplet excitons, which can be obtained by measuring the triplet-induced absorption spectrum of the material under study blended with a triplet sensitizer, and known spectra for charged species, by comparing the TA spectra to the absorption spectra of chemically oxidized or reduced samples. 
A

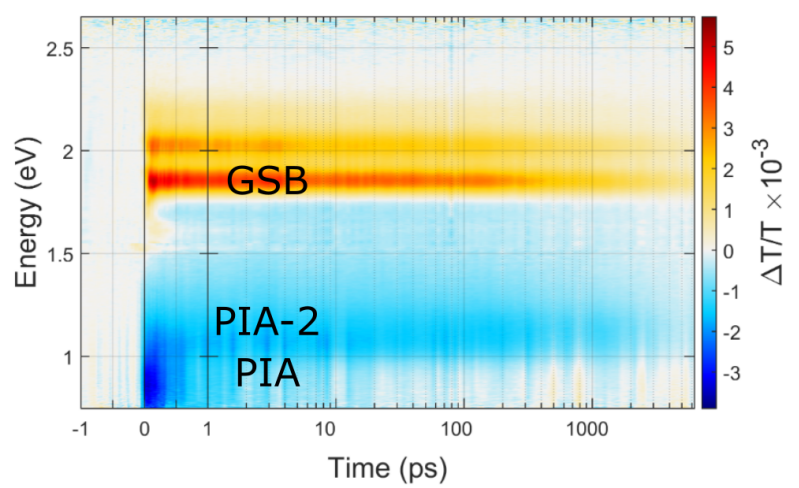

B

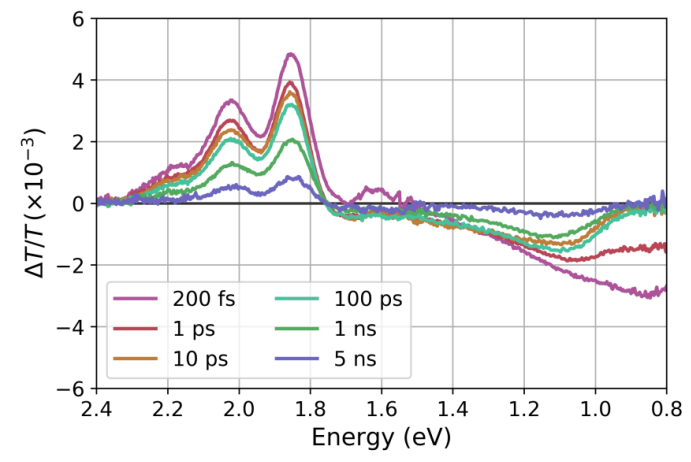

C

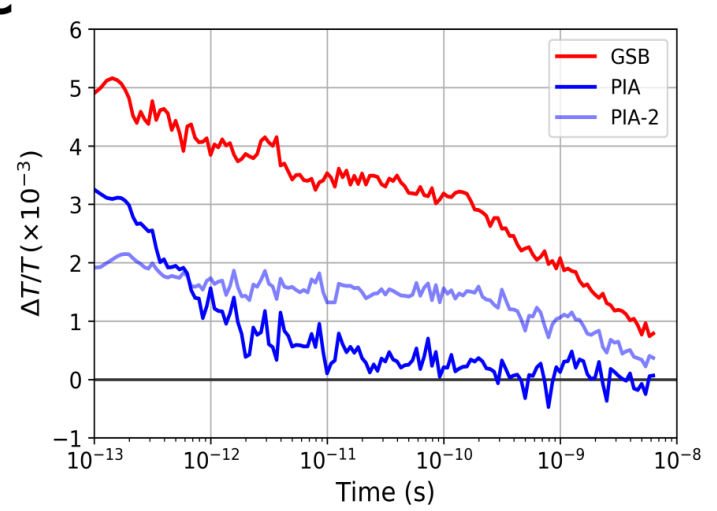

Figure 3.3: A) A typical TA surface, exhibiting a GSB and two PIA features, namely PIA and PIA-2. B) Spectral slices of the TA surface at indicated time delays. C) Kinetic profiles obtained by direct integration over the indicated spectral regions. 
In order to carry out a more detailed analysis of the data, including data modelling of the dynamics of certain species, we need to ensure that the overlapping features in the TA surface are disentangled. In some cases, it is possible to obtain clean dynamics for individual species by direct integration over spectral regions, as explained earlier. However, in most cases the spectral features are overlapping, and further manipulation of the data is required to separate the individual contributions. We achieve such deconvolution by using the powerful technique known as Multivariate Curve Resolution which is described in the following section.

\subsection{Multivariate curve resolution}

As noted previously, TA data is arranged in a multi-component matrix $M$, involving contributions from different photo-active transient species. The data analysis we implement here is based on a bilinear model, i.e. the decomposition of the data set into the product of two matrices, the spectrum of the transient species $S^{T}$ and their time-dependent concentration profiles or kinetics $C$, according to Equation 3.4, where $k$ is the number of pure species. A representation of such decomposition is shown in Figure 3.4 for a system composed by two transient species.

$$
M=C \cdot S^{T}+E=c_{1} \cdot s_{1}^{T}+c_{2} \cdot s_{2}^{T}+\ldots c_{k} \cdot s_{k}^{T}+E
$$




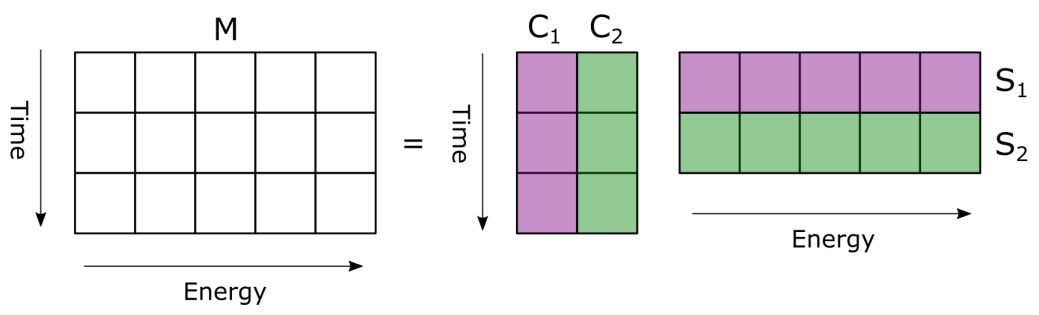

Figure 3.4: Bilinear decomposition of the multicomponent data set $\mathrm{M}$, composed by two transient species.

The number of independent components in a data set can be determined via either principal component analysis or via a factorization of the data matrix known as singular value decomposition (SVD). SVD yields the following product of three matrices:

$$
M=U S V
$$

where the columns of $\mathrm{U}$ and the rows of $\mathrm{V}$ are orthogonal and $\mathrm{S}$ is a diagonal matrix. The diagonal elements of $S$ are the square roots of the eigenvalues of $M M^{T}$ or $M^{T} M$ in descending order, which are known as singular values. Examination of the magnitude of the singular values allows us to distinguish components related to physical contributions, for which the singular values are large, from components related to noise, for which singular values are small and similar among them. It is worth mentioning that this decomposition is completely abstract and the matrices $\mathrm{U}$ and $\mathrm{V}$ have no chemical or physical meaning [90]. Therefore, physical constraints related to the systems of interested need to be incorporated into the analysis.

Multivariate curve resolution (MCR) is a method developed by Tauler and coworkers which solves Equation 3.4 through an iterative linear least square 
algorithm and does not require a priori knowledge on the kinetics of the system [91-93]. Multivariate Curve Resolution by Alternating Least Squares (MCR-ALS) calculates concentration $C$ and pure spectra $S^{T}$ matrices by optimally fitting the experimental data matrix M. MCR-ALS was implemented by the authors in the MATLAB environment with an intuitive graphical interface and it is available for download, to be used by anyone with a MATLAB license. Once the number of independent components is defined via SVD, initial estimates of $C$ or $S^{T}$ can be obtained using Evolving Factor Analysis (EFA) which uses a forward and backward going SVD on a sequence of submatrices and use the singular values to estimate how populations of excited-states evolve with time [94]. Once the initial concentrations are calculated, the optimisation method is carried out following the steps in Figure 3.5. The Figure shows how constraints are applied to spectra and concentration profiles in each iteration.

It is important to note that, when disentangling multiple components from the transient data, the solutions obtained via MCR are not unique, since different solutions might be mathematically equivalent, i.e. they might be able to explain the data equally well. Such mathematical limitation, intrinsic to the MCR-ALS method, is due to rotational and intensity ambiguities of the solutions. The most straightforward way to restrict the range of possible solutions is by applying constraints. Some constraints that we commonly use are non-negativity for concentration profiles, unimodality in spectra, and equality constraints, by including the pure spectrum of one or more of the transient components. Ambiguities can be related to different issues, such as intensity and rotational ambiguities. This can be demonstrated by re-writing Equation as:

$$
\begin{gathered}
\mathbf{M}=\mathbf{C}\left(\mathbf{R R}^{-\mathbf{1}}\right) \mathbf{S}^{\mathbf{T}}+\mathbf{E} \\
\mathbf{C}^{\prime}=\mathbf{C R}
\end{gathered}
$$




$$
\mathbf{S}^{\prime \mathbf{T}}=\mathbf{R}^{-1} \mathbf{S}^{\mathbf{T}}
$$

where the matrices $\mathbf{C}^{\prime}$ and $\mathbf{S}^{\prime}$ are rotationally equivalent to the real $\mathbf{C}$ and $\mathbf{S}^{\prime}$ and the decomposition of $\mathbf{M}$ based on $\mathbf{C}^{\prime}$ and $\mathbf{S}^{\prime}$ leads to the same residuals as the one based on $\mathbf{C}$ and $\mathbf{S}$. If $\mathbf{R}$ is a diagonal matrix the ambiguities are simply affecting the scale of the solutions, and this is known as an intensity ambiguity, otherwise the shape of both spectra and concentrations profiles are affected, which is known as a rotational ambiguity [93]. As discussed before, we apply constraints to restrict the space of possible solutions and retrieve physically meaningful pairs of spectrum and kinetics.

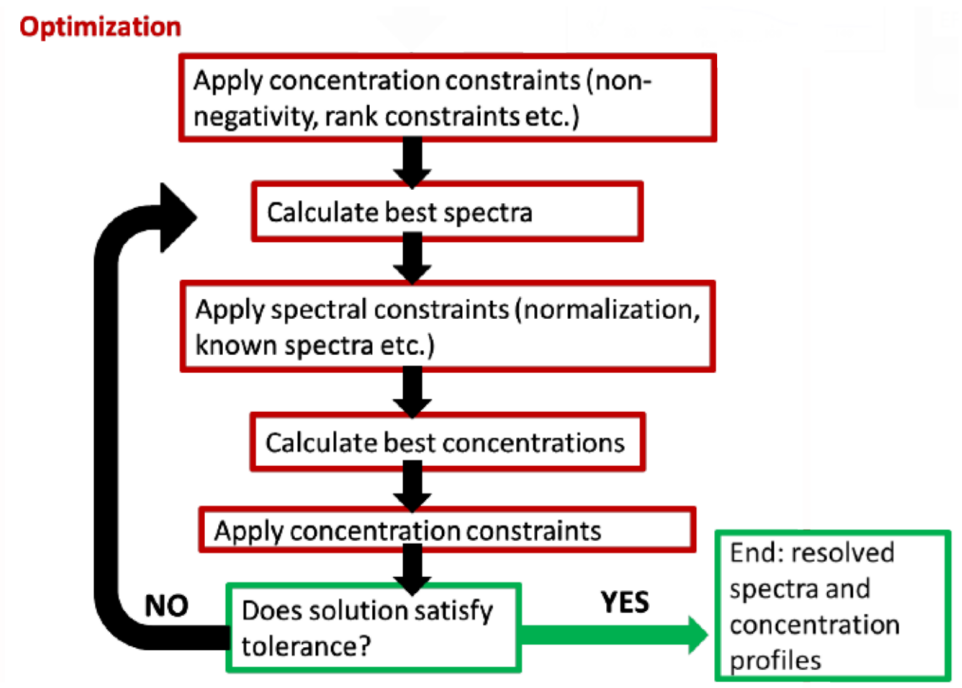

Figure 3.5: The optimization procedure performed by the MCR-ALS method, which involves the application of constraints and an iterative linear least square algorithm. Figure modified from Reference [94].

The MCR-ALS approach has been widely used for the analysis of TA data in the OPV community, enabling the separation of different excited states, such 
as excitons, charges and triples states [95-98]. In addition to the limitations of the method due to the mathematical ambiguities explained before, which can be tackled by the use of constraints, it is worth mentioning that, the MRC-ALS algorithm is not able to differentiate individual species when their sprectra are very similar. This has been found to be the case of bound CT states and free separated charges in OPV thin films, which exhibit the same mean cross-section. However, once the overall charge component is isolated from other excited states via MCR-ALS, the behavior of CT states and separated charges may be isolated via fluence-dependent measurements, since their dynamics scale differently with charge density $[61,63]$. 


\section{Chapter 4}

\section{How atom substitution affects the}

\section{photophysics of low-bandgap copolymers for OPV cells}

\subsection{Overview}

A widely used approach to synthesise low-band-gap polymers is based on an alternating donor-acceptor (D-A) or push-pull design that comprises the use of alternating electron-rich and electron-deficient moieties as repeating units in the main chain of the polymer [39]. The ability to systematically vary these units within the chemical structure has been exploited to manipulate electronic and optical properties, resulting in materials with narrow optical energy gaps and high absorption coefficients, allowing a more efficient harvesting of the solar spectrum. However, small changes, such as the replacement of a single atom in the donor or acceptor unit, have shown to cause large variations in the photovoltaic parameters, which cannot be explained considering only the variations in the optical band-gap [40,99-103]. 
In this work, we study a family of polymers derived from the low-band-gap alternating copolymer, poly5,6-bis(octyloxy)-4-(thiophen-2- yl)benzo[c]-1,2,5thiadiazole (PTBT) which was previously found to produce high performance photovoltaic devices when blended with fullerene derivatives [87]. We explore the photophysics of a family of related polymers, where the sulphur atom in the thiadiazole unit was substituted with oxygen or selenium. The photovoltaic characteristics show that the power conversion efficiency (PCE) in a PTBSe:PC ${ }_{61} B M$ device is $94 \%$ lower than that in the analogous PTBT:PC ${ }_{61} \mathrm{BM}$ device [87] under the same preparation conditions. Interestingly, previous studies reported in the literature show that the substitution of sulfur by selenium results in an improvement in the photovoltaic performance in some systems $[100,101,103]$, but it has a detrimental effect for other systems [102].

Our ultrafast transient absorption spectroscopy (TAS) measurements on the neat polymers and the polymer:fullerene blends explain the inferior photovoltaic performance of PTBO and PTBSe with respect to the original PTBT polymer. In PTBO: $\mathrm{PC}_{61} \mathrm{BM}$, we find that $33 \%$ of excitons are promptly quenched generating charges, with the reminder $67 \%$ needing to diffuse to the interface to undergo charge transfer. This suggests that the morphology of this blend is not as finely intermixed as the original PTBT blend, where nearly all excitons were quenched in an ultrafast time scale. Also in contrast with the PTBT system, recombination dynamics in $\mathrm{PTBO}: \mathrm{PC}_{61} \mathrm{BM}$ shows that geminate recombination, i.e. recombination of geminate pairs at the donor:acceptor interfase subsequent to the exciton quenching process, is a severe limiting factor in this system. In the case of PTBSe:PC ${ }_{61} \mathrm{BM}$, we find that all excitons are quenched in an ultrafast time scale, which, in addition to its broad and unstructured steady-state absorption, suggests a morphology that is more strongly intermixed than in the PTBT system. Exciton quenching is accompanied by ultrafast charge formation, 
followed by the formation of triplet excitons in the nanosecond time scale, due to enhanced intersystem crossing by incorporating the heavy atom selenium into the chemical structure. In both PTBO and PTBSe systems, strong recombination of geminate CT pairs is identified as the major limiting factor, through the ${ }^{1} C T \rightarrow G S$ pathway in the case of PTBO:PC ${ }_{61} \mathrm{BM}$ and via the ${ }^{3} C T \rightarrow^{1} T$ pathway in the case of PTBSe:PC ${ }_{61} \mathrm{BM}$. Our results are consistent with the literature, where it has been shown, in a closely related polymer, that the sulfur-containing compound has a more favorable intermolecular packing, resulting in better morphology and charge carrier transport, properties that are essential for efficient charge separation and extraction [102]. Thus, we speculate that unfavorable packing of the $\mathrm{PTBO}$ and PTBSe polymers at the interfaces with $\mathrm{PC}_{61} \mathrm{BM}$ results in higher geminate recombination rates for these systems. Additionally, in the case of PTBSe:PC ${ }_{61} \mathrm{BM}$ the heavy atom effect facilitates the formation of triplet excitons, which is identified as a major energy loss channel.

\subsection{Background}

\subsubsection{Single-atom substitution in low-band-gap copolymers}

As noted in the overview of this chapter, the replacement of a single atom in the donor or acceptor units of low-band-gap copolymers has shown to cause large variations in photovoltaic performance, which cannot be explained considering only the variations in the optical band-gap. The literature suggests that the effect of substituting one atom of sulfur by selenium in the chemical structure depends upon the system. In Reference [101] the authors investigate how the substitution of a sulfur atom by selenium in the thiadiazole unit of a low-band-gap small molecule affects the device optoelectronic properties and photovoltaic performance. The study shows that the selenium containing 
variant leads to a higher efficiency in a standard organic photovoltaic architecture, when combined with $\mathrm{PC}_{71} \mathrm{BM}$ and thermally annealed. The authors emphasise that this heteroatom engineering approach for the development of higher efficiency organic solar cells needs to consider details such as solubility, solid-state crystallization, packing, and the resulting morphology within the device, and not simply electronic considerations. Similarly, in Reference [103] the authors studied the substitution of a sulfur atom by selenium in the accepting units of D-A alternating polymers. Such substitutions result in remarkable changes in the optoelectronic properties of polymers, with the benzoselenadiazole (BSe) polymers showing enhanced photovoltaic performances than benzothiadiazole (BT) based polymers, with a strong dependence upon the processing conditions and morphology of the active layers. However, the improved photovoltaic performance in the variants containing selenium is not a general rule. In reference [102] the authors studied the effects of systematic sulfur/selenium substitutions into both donor and acceptor units using complementary Raman spectroscopy, density functional theory, and X-ray diffraction as characterisation tools. The authors demonstrate that, in each case, the heavy atom substitution is detrimental to photovoltaic performance, mainly due to unfavourable intermolecular packing in thin films and poor charge carrier mobility.

\subsubsection{Previous study on PTBT}

The low-band-gap alternating copolymer poly5,6-bis(octyloxy)-4-(thiophen2-yl)benzo[c]-1,2,5- thiadiazole (PTBT), which is the parent structure in this study, was previously found to produce high performance photovoltaic devices when blended with fullerene derivatives [87]. The work was carried out by Gallaher et al. on a series of closely related alternating copolymers: PTTBT, 
PDTBT, and PTBT, which have small variations in polymer backbone curvature, which was used to alter the component mixing in polymer:fullerene blends by altering the miscibility of fullerene. This yields the controlled formation of either 2- or 3-phase morphology over the series of polymer materials. The authors analysed charge dynamics in 3-phase blends compared with binary counterparts and linked these dynamics to free charge yields [87]. In particular for PTBT the copolymer, the processes of charge generation and recombination studied via TAS measurements by Gallaher et al. are summarized as follows:

- Nearly all excitons are quenched within the $200 \mathrm{fs}$ time resolution of the experiment, suggesting a finely intermixed blend morphology that does not require excitons to diffuse to reach an interface.

- Efficient formation of hole polarons upon exciton quenching is accompanied with the retention of the GSB.

- The hole polaron band shifts to lower energy over time, and the relative intensity of the 0-0 band in the GSB increases, which was attributed by the authors to the migration of excitations from disordered polymer segments to ordered chains with larger delocalisation.

- The GSB and charge intensity are retained in the nanosecond timescale.

Here, we explore a new family of related polymers based on PTBT, where the sulphur atom in the thiadiazole unit was substituted with oxygen or selenium in order to investigate how atom substitution affects the photophysics in this family of related low-bandgap copolymers for photovoltaic cells. Molecular structures of these materials are depicted in figure 4.1. 


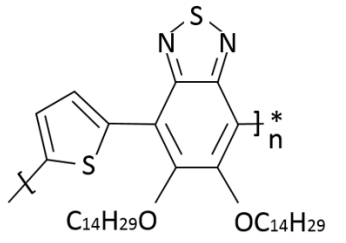

PTBT

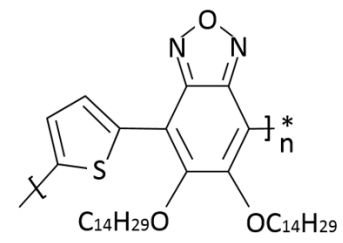

PTBO

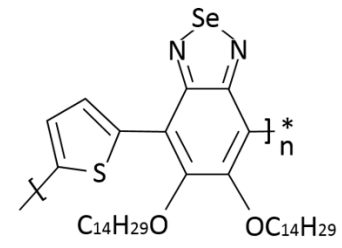

PTBSe

Figure 4.1: Molecular structures of the low-band-gap copolymers PTBT, PTBO and PTBSe under study.

\subsubsection{The role of spin in organic photovoltaic devices}

In systems featuring heavy atoms, such as selenium, triplet exciton formation via intersystem crossing (ISC) is enhanced due to strong spin-orbit coupling, and thus, we expect that electronic triplet states play a significant role in the optoelectronic characteristics of such devices [104-109]. As described in Section 2.9 of this thesis, the absorption of a photon with an energy above the optical bandgap in an OPV active layer, leads to the formation of localised singlet excitons. When excitons reach the donor:acceptor heterojunction, the charge transfer process takes place, with positive polarons being formed in the donor phase and negative polarons being formed in the acceptor phase. The process of dissociation of the polaron pairs into separated charges, that could potentially be extracted at the electrodes of the device, competes with the decay of the CT states (or polaron pairs) to the ground state. In fact, even when charges have been completely dissociated, in their way to the electrodes they may undergo undesirable nongeminate recombination, resulting in a re-creation of CT states. Because the electron and the hole orbitals in a CT state are separated in space, the splitting between singlet and triplet CT state energies is small [110] and in low-band-gap polymer donor materials, the energy of the triplet exciton $\left({ }^{1} \mathrm{~T}\right)$ is typically lower than the energy of the CT states. Therefore, the decay of ${ }^{3} \mathrm{CT}$ into 
${ }^{1} \mathrm{~T}$ is energetically favourable. Subsequently, triplet excitons either decay to the ground state or annihilate via triplet-triplet annihilation (TTA) or triplet-charge annihilation (TCA). Figure 4.2 shows a state diagram representing the different photophysical processes [104].

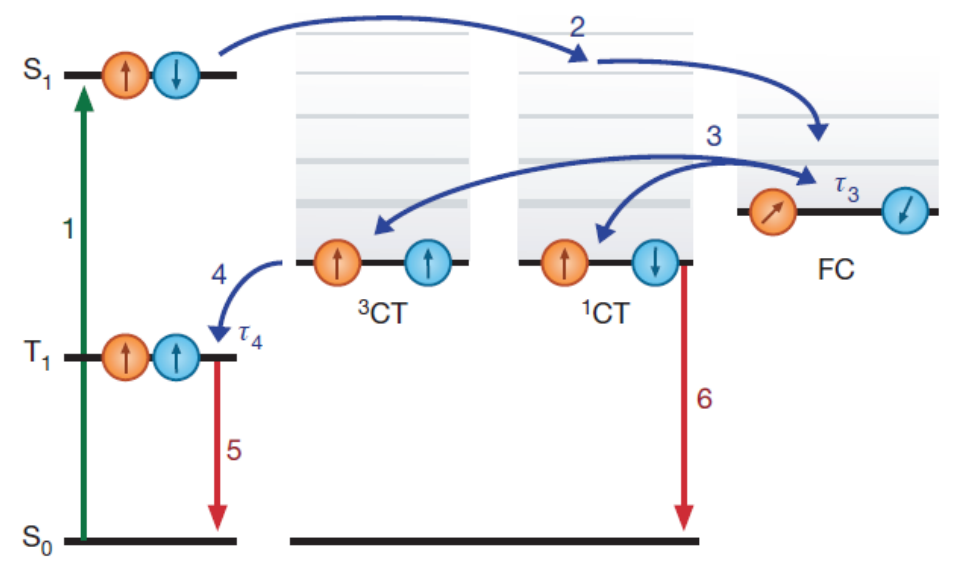

Figure 4.2: State diagram representing the various photophysical processes in an OPV device. The Figure is reproduced with permission from Refence [104].

The role of spin in charge recombination was studied experimentally by Rao et al. who demonstrated that electron-hole encounters lead to the formation of CT states with singlet and triplet character [104]. Their time-resolved spectroscopic study in polymer:fullerene systems where the energy of the triplet exciton in the polymer is lower than the energy of the CT state, shows that the relaxation of ${ }^{3} \mathrm{CT} \rightarrow{ }^{1} \mathrm{~T}$ can be suppresed by wavefunction delocalization, resulting in CT re-dissociating into free charges. In this way, the authors could explain the high external quantum efficiencies despite the high rate of electron-hole encounters in the bulk-heterojunction. The authors applied a kinetic model in a PIDT-PhanQ:ICBA blend and extracted a high triplet-charge annihilation rate, which explains why this is a loss channel. Triplet formation is observed in ICBA and ICMA blends at room temperature but is out-competed by dissociation 
back to free charges in the 1:3 PIDTPhanQ: $\mathrm{PC}_{60} \mathrm{BMblend}$. Additionally, in the efficient 1:3 PIDT-PhanQ:PC ${ }_{60} \mathrm{BM}$ blend triplets are formed at low temperatures $(<240 \mathrm{~K})$, but not at room temperature, suggesting that there is a thermally activated process competing with the relaxation to ${ }^{1} \mathrm{~T}$, which is dissociation of ${ }^{3} \mathrm{CT}$ back to free charges. The authors concluded that large aggregates in PCBM assists charge separation due to delocalization of the electron wave function over the aggregates.

Within the same framework, Chow et al. used the same model for the triplet dynamics to characterize the effect of morphology on recombination of PCPDTBT:PC ${ }_{70} \mathrm{BM}$ blends through the use of solvent additives [105]. The authors found that without additive, the IQE was $48 \%$ and $16 \%$ of excitations were decaying via bimolecular triplet formation. With the additive treatment however, crystallinity and carrier mobility were improved and the IQE increased to $65 \%$, but in contrast with the PIDT-PhanQ:PC ${ }_{60} \mathrm{BM}$ blend studies by Rao et al. [104], bimolecular triplet formation was increased to 35\%, suggesting that the presence of PCBM aggregates is not a sufficient condition to avoid recombination via triplets. The authors pointed out that the nature of PCBM clusters (size, crystallinity, etc.) is likely to dependent on the donor polymer.

A detailed kinetic study based on the Onsager-Braun model including back electron transfer was implemented by Shoaee et al. [108]. The authors show that suppression of both of the parallel loss channels of singlet and triplet states via nongeminate recombination can be achieved through increasing the dissociation rate of the CT states. The authors explain that due to the limitations to improve charge extraction in OPV devices, due to their intrinsic low free carrier mobilities, the possibility of reducing bimolecular recombination can be key for achieving high charge collection efficiencies, providing sufficiently long charge 
carrier lifetimes to be collected at the electrodes.

Interestingly, the competition between the dissociation of CT states and their recombination to an energetically favorable triplet state has been emphasized in a recent article which shows that in the systems with very efficient charge generation and very fast CT dissociation, free carrier recombination is strongly suppressed, regardless of the spin factors [109].

\subsection{Results and discussion}

\subsubsection{Device characteristics}

The photovoltaic parameters of the OPV devices under study in a conventional device structure are summarized in Table 6.1. We notice important variations in the $J_{s c}$ and $F F$ parameters, with the highest values in the device featuring the PTBT polymer and the lowest values when featuring the PTBSe polymer. $J_{s c}$ decreases by $83 \%$ and $F F$ decreases by $64 \%$ when PTBT is replaced by PTBSe, resulting in the overall power conversion efficiency (PCE) in the PTBSe:PC ${ }_{61} \mathrm{BM}$ device being $94 \%$ lower than that in the PTBT:PC ${ }_{61}$ BM device.

Table 4.1: Photovoltaic characteristics in the devices under study.

\begin{tabular}{cccc}
\hline & PTBT:PC $_{61}$ BM & PTBSe:PC $_{61}$ BM & PTB0:PC $_{61}$ BM \\
\hline$V_{o c}$ & 0.861 & 0.878 & 0.747 \\
\hline$J_{s c}$ & 11.7 & 1.90 & 3.28 \\
\hline FF & 0.703 & 0.253 & 0.432 \\
\hline PCE & 7.07 & 0.422 & 1.06 \\
\hline
\end{tabular}




\subsubsection{UV-Vis steady state absorption}

Before discussing our TAS measurements, we will examine the steady state absorption of the materials. UV-Vis steady state absorption spectra of the neat polymer donors $\mathrm{PTBO}$ and PTBSe and their blends when matched with $\mathrm{PC}_{61} \mathrm{BM}$ were obtained in a Cary $50 \mathrm{UV}$-Vis Spectrophotometer and are shown in Figure 4.3. Identical spectra were measure before and after our TA measurements, confirming that no degradation occured during the experiments. In PTBO we observe predominant absorption peaks at $650 \mathrm{~nm}$ and $610 \mathrm{~nm}$, assigned to $0-0$ and $0-1$ vibronic transitions. The ratio between these peaks intensities has been related to different types of aggregation in the polymeric phase, i.e. J-aggregates ( $\mathrm{J}$ for Jelly) and $\mathrm{H}$-aggregates ( $\mathrm{H}$ for hypsochromic) as we discussed in Chapter $2.5[83,111,112]$. The PTBO film exhibits a 0-0/0-1 ratio larger than 1 , consistent with a predominant contribution from J-aggregates due to intrachain throughbond interactions. When blended with the acceptor material the 0-0/0-1 ratio is decreased which can be explained by the disruption of the polymer order by the addition of $\mathrm{PC}_{61} \mathrm{BM}$ [87]. In PTBSe the absorption spectra is broad and unstructured, which can be explained by H-type aggregates due to interchain Coulombic interactions, and the incorporation of $\mathrm{PC}_{61} \mathrm{BM}$ results in a blue-shift of the spectra. TAS experiments were performed with an excitation wavelength of $650 \mathrm{~nm}$, indicated with a vertical line in the figure. 


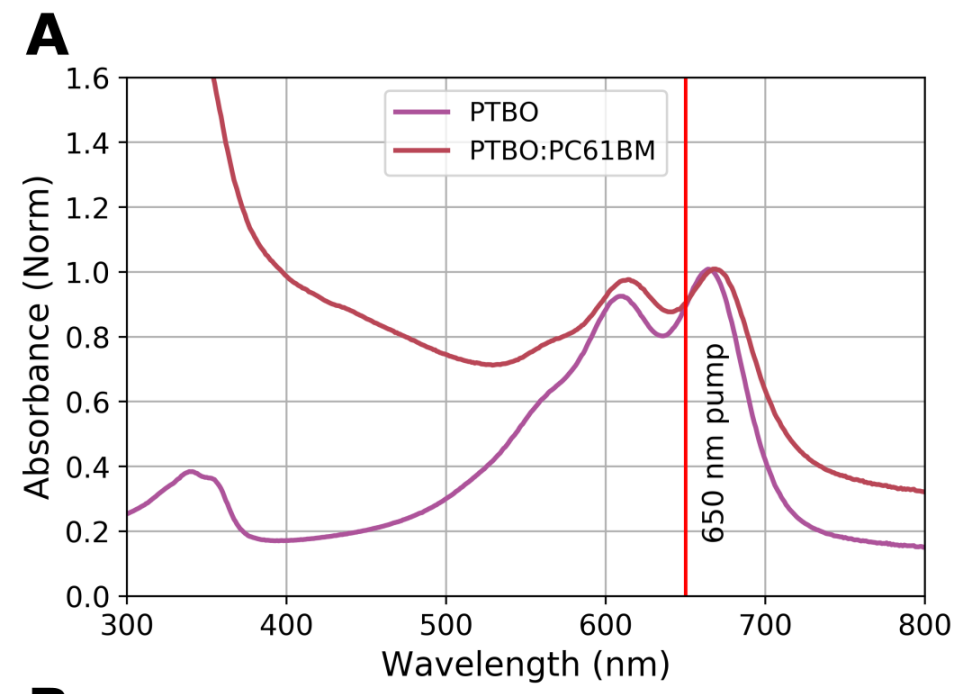

B

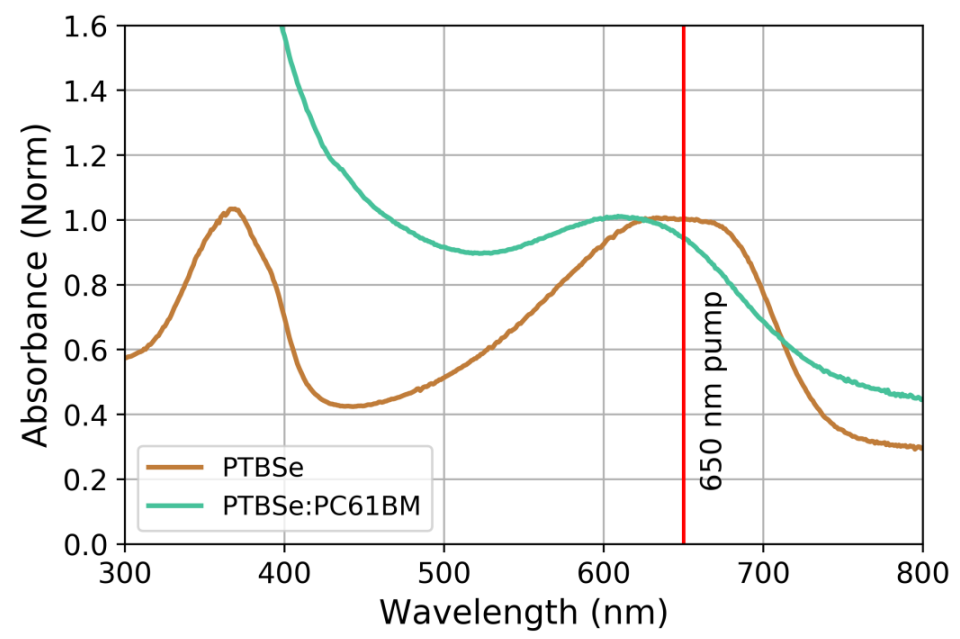

Figure 4.3: UV-visible absorption spectra of neat polymer donors A) PTBO and B) PTBSe, along with their fullerene blends.

\subsubsection{Transient absorption spectroscopy}

We performed TAS measurements at room temperature using the experimental setup described in Section 3.3 of this thesis. Pump pulses were obtained by tuning a parametric amplifier (TOPAS) to the desired wavelength and using 
a retroreflector mounted in a mechanical delay stage to obtain pump-probe delays up to $6 \mathrm{~ns}$. The spectral window of the broadband probe pulses covers the range $0.77-3.1 \mathrm{eV}$ (equivalent to $400-1600 \mathrm{~nm}$ ), allowing us to monitor dynamics of the photo-generated transient species.

\subsubsection{Neat PTBO and PTBO:PC ${ }_{61}$ BM}

\section{Neat PTBO}

Our TAS data on a PTBO neat film is shown in Figure 4.4 A as spectral slices at indicated time delays. The TAS features we observe are the GSB in the wavelength region $1.8-2.4 \mathrm{eV}$, a weak SE feature at $1.5-1.7 \mathrm{eV}$ and a PIA feature in the wavelength region $0.8-1.4 \mathrm{eV}$. Singular value decomposition of the data matrix returns singular values suggesting that a single component is sufficient to explain the data. Therefore, all features decay with the same kinetics, which is confirmed by identical integrated kinetics of the GSB and PIA features, as shown in Figure 4.4 B. In the same figure we show that the GSB (and PIA) kinetics can be modelled by a single exponential model. The equivalent decay of all features, together with the presence of the SE feature due to the $S_{1} \rightarrow S_{0}$ transition, supports our assignment of the PIA band as an exciton-induced absorption signal $[113,114]$. A nonlinear least squares fit of the exponential decay in Figure $4.4 \mathrm{~B}$ indicates an exciton half-life of approximately $70 \mathrm{ps}$, which is the order of magnitude found in other conjugated polymers measured via transient absorption or fluorescence spectroscopy [72]. 

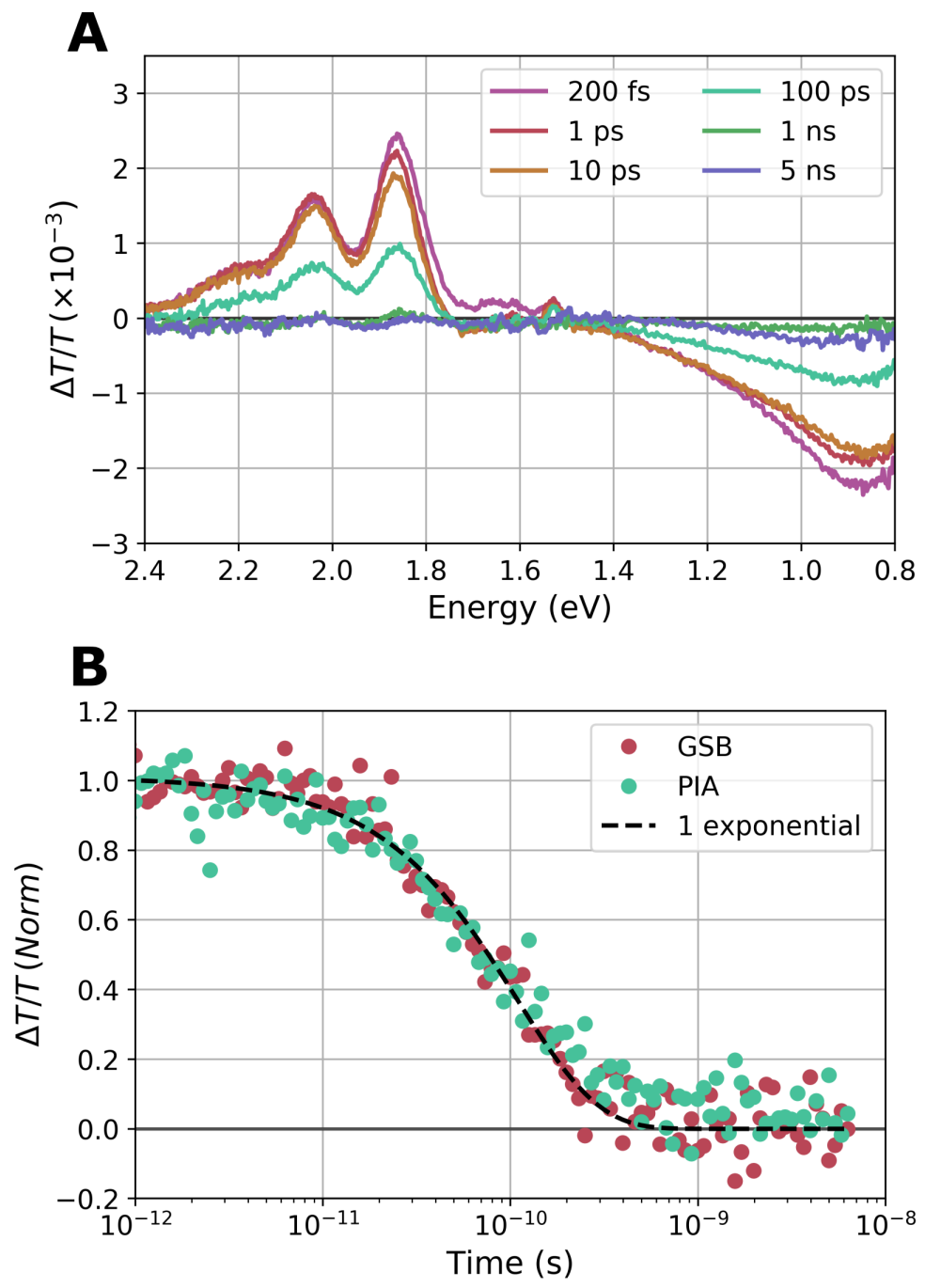

Figure 4.4: A) Spectral slices of the TAS data measured in a PTBO neat film at indicated time delays, and B) integrated kinetics of GSB and PIA features. Such kinetics can be modelled by a single exponential model.

\section{PTBO:PC ${ }_{61}$ BM}

In Figure 4.5 we show spectral slices of the TAS data measured in a PTBO:PC ${ }_{61} \mathrm{BM}$ blend film at indicated time delays, along with the excitonic 
spectra previously measured in the neat PTBO film. We note the matching overlap between the spectrum of the PTBO exciton and the early time spectra (at 200 fs time delay) in the blend film, however we observe that the ratio of the exciton PIA to the GSB in the blend is lower than such ratio in neat PTBO. According to such variation in PIA/GSB ratio, 33\% of excitons are promptly quenched after $200 \mathrm{fs}$, with the simultaneous emergence of a new $\mathrm{PIA}_{2}$ peak in the wavelength region $1.0-1.2 \mathrm{eV}$. This suggests that $33 \%$ of excitons are generated at very close proximity to the donor:acceptor interfase. However, the morphology of this blend is not as finely intermixed as the PTBT blend, where nearly all excitons were quenched in an ultrafast time scale. In the PTBO system, prompt charge generation is followed by further growth of the $\mathrm{PIA}_{2}$ feature in the region around $1.0-1.2 \mathrm{eV}$ at the expense of the exciton PIA, which we attribute to excitons reaching the donor:acceptor interface and undergoing charge transfer. In the $\mathrm{PIA}_{2}$ region we note an apparent shift of the peak towards slightly higher energies (i.e. a blue-shift) as time progresses. However, by decomposing the data matrix into independent contributions, we find that only two components are necessary to explain the data. Thus, we attribute the apparent shift in the $\mathrm{PIA}_{2}$ peak to the overlap of this signal with the exciton PIA at early times. The appearance of PIA 2 upon exciton quenching is attributed to charge transfer from the polymer to the fullerene and the concomitant appearance of charges. Another plausible explanation for this new feature could be the formation of triplet states in the polymer. However, it has been noted in previous works that in donor:acceptor systems, the charge transfer rate is much faster than that of triplet formation [113-116]. In this way, we attribute $\mathrm{PIA}_{2}$ to a charge-induced absorption feature originated from the absorption of hole polarons in the polymer. 


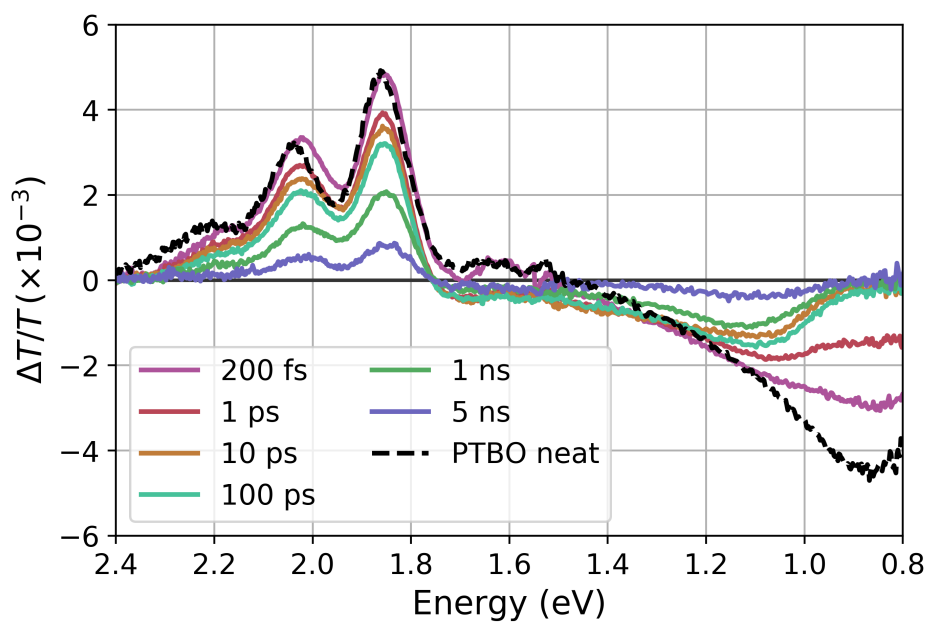

Figure 4.5: Spectral slices of the TAS data measured in a PTBO:PC ${ }_{61} \mathrm{BM}$ blend film at indicated time delays. Excitonic spectra measured in the neat PTBO film is superimposed to show its overlap with the early time spectra in the blend film.

The individual contributions from excitons and charges to the overall TA signal were obtained by using the soft-modelling algorithm known as Multivariate Curve Resolution by Alternating Least Squares (MCR-ALS) introduced in detail in Section 3.4 of this thesis [91-93]. Such decomposition allows us to recover the spectra and kinetics of the transient species, as long as they exhibit differentiable kinetics over time. As mentioned in Section 3.4, the outputs of this decomposition are not unique and additional information must be provided to obtain results with physical meaning. To achieve this, we used the following constraints: i) that kinetic profiles can not be negative, ii) that PIA signals can not be positive, and iii) we introduced the spectra of neat PTBO as a spectral mask, i.e. a fixed spectra that is forced to match one of the MCR-ALS components. The resulting MCR kinetics of excitons and charges are presented in Figure 4.6 A and B for experiments using pump fluences of $1.5 \mu \mathrm{J} / \mathrm{cm}^{2}$ and $6 \mu \mathrm{J} / \mathrm{cm}^{2}$. In Figure 4.6 C we include the integrated kinetics of the GSB normalised to one at a 
time delay of $200 \mathrm{fs}$. Since depopulation of the ground state is associated with the population of all excitations, including excitons and charged species, the GSB serves to quantify the population of excitons $E(t)$ and charges $C(t)$ over time, according to a linear combination such as $G S B(t)=E(t)+C(t)$ [87]. In this way, we are able to transform exciton and charge kinetics into normalized populations, shown in Figure 4.6 C. 
A
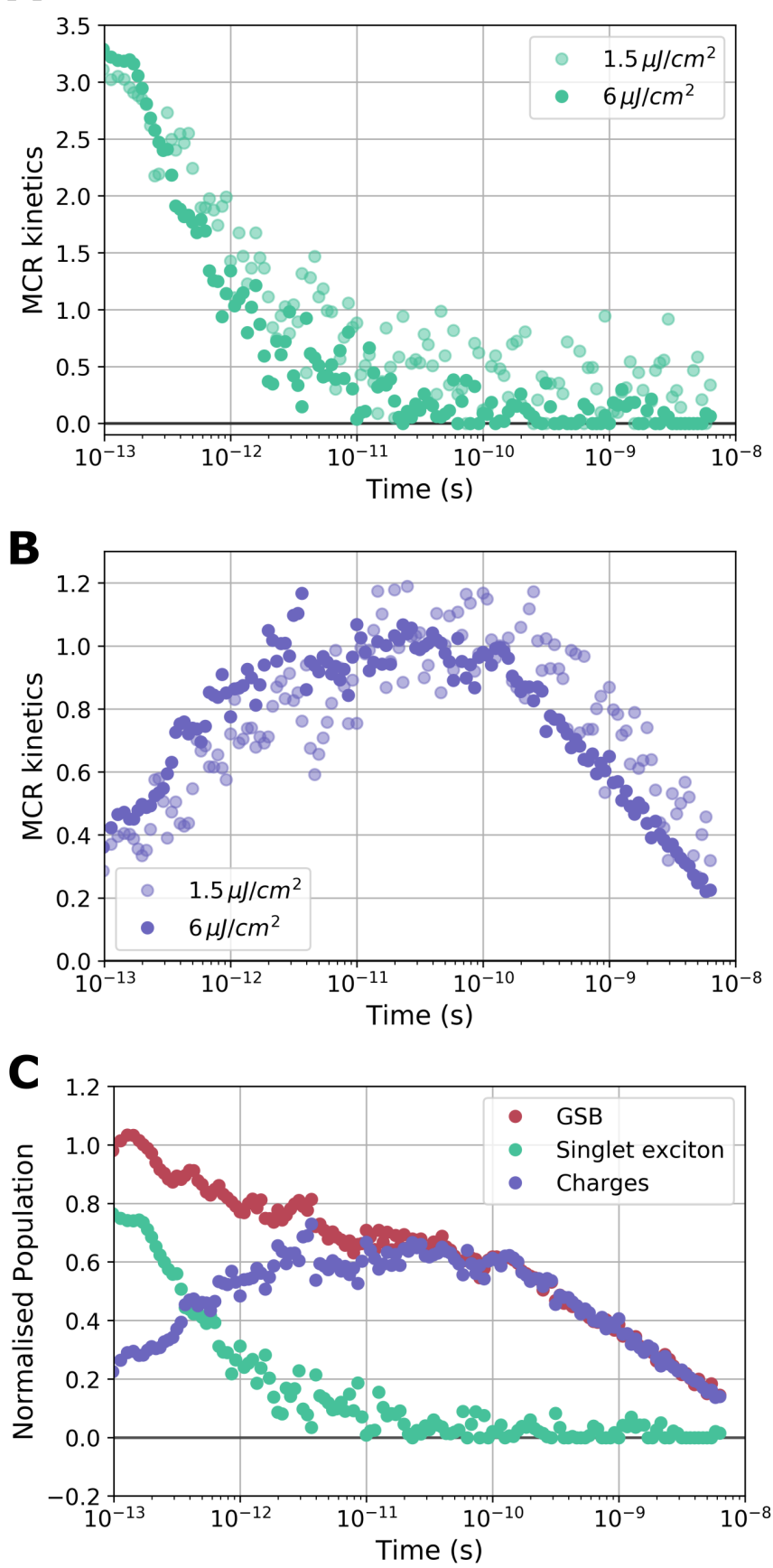

Figure 4.6: $M C R$ kinetics of $\mathrm{A}$ ) excitons and $\mathrm{B}$ ) charges in $\mathrm{PTBO}: \mathrm{PC}_{61} \mathrm{BM}$ at two different pump fluences. C) Normalised population of excitons and polymer hole polarons based on the integrated intensity of ground state bleach (GSB). 
We observe from Figure 4.6 C that excitons in the PTBO:PC ${ }_{61} \mathrm{BM}$ blend decay considerably faster than in neat PTBO, with a half-life below $1 \mathrm{ps,} \mathrm{compared}$ with the intrinsic exciton lifetime of 70 ps in neat PTBO, suggesting that excitons are rapidly quenched at the donor:acceptor interface before decaying to the ground state. However, at time delays where charges are photo-generated via excitons, the intensity of the GSB is not retained, as we would expect, and possible explanations involve exciton decay processes such as exciton-exciton or exciton-to-charge annihilation. According to Figure 4.6 A, exciton decay does not depend upon pump fluence, and thus we rule out the process of excitonexciton bimolecular annihilation. A more likely explanation is the decrease in GSB intensity caused by exciton-to-charge annihilation, due to the temporal overlap of exciton and charge species with sufficiently high density. [117].

Figure 4.6 C additionally indicates that the normalised charge population exhibits a pronounced decay at time delays above 30 ps, and Figure 4.6 B shows that such charge decay is practically fluence-independent. Such characteristics are, according to the literature, indicatives of strong geminate recombination of bound charge pairs to the ground state, which prevents their split into separated charges [118]. The early timescale of geminate recombination is associated with the fact that polaron pairs (also known as CT pairs) are formed directly after exciton dissociation and recombine subsequently in a process that depends on the local structure at the donor:acceptor boundary, as explained by Muller et al. in Reference [119] for polymer:fullerene systems and by Hodgkiss et al. in Reference [113] for polymer:polymer systems. Since geminate recombination involves bound pairs exclusively, the decay rate does not depend on the density of excited states and therefore, does not depend on the excitation fluence. Avoiding fast geminate recombination is essential for efficient charge separation and eventually extraction at the electrodes [120] and thus, we derive from our results 
that geminate recombination is the major limiting factor in this blend system.

The main loss pathway that we have identified in the PTBO:PC ${ }_{61} \mathrm{BM}$ is illustrated in the state diagram in Figure $4.12 \mathrm{~B}$, along with the state diagrams of the PTBSe system studied here and of the original PTBT system. Dominant pathways are represented with bold colours, and less efficient pathways in dashed colours.

\subsubsection{Neat PTBSe and PTBSe:PC 61 BM}

\section{Neat PTBSe}

Figure 4.7 shows spectral traces of the TAS data measured in a PTBSe neat film at indicated time delays. Due to low signal-to-noise ratio in this measurement we can not access the full range of wavelengths available in our probe pulse, but nevertheless we distinguish the GSB feature, SE due to singlet exciton decay and two PIA features: a broad PIA that peaks within the spectral region $0.8-1.0 \mathrm{eV}$ and a subsequent $\mathrm{PIA}_{2}$ peak within the region $1.0-1.2 \mathrm{eV}$. This is in contrast with the neat PTBO data discussed earlier and with the measurements in neat PTBT reported in the literature [87], where a single PIA feature was measured upon exciton decay. However, additional excited species can be generated in neat materials, including charged species and triplet excitons. Previous work reported in the literature shows that neat conjugated polymers can exhibit charge photogeneration, primarily obtained from the breakup of intrachain excitons, and that long-lived charge generation can occur at the interface between amorphous and crystalline domains of the polymer [121]. On the other hand, the formation of triplet excitons in neat materials can occur via intersystem crossing (ISC) from singlet excitons, which depends on spin-orbit coupling and on the vibrational overlap between the states involved. Spin-orbit coupling increases strongly 
with the atomic number and therefore is enhanced by incorporating heavy atoms into the chemical structure. In this way, it is not surprising to detect the conversion from singlet to triplet excitons in this material containing the heavy atom selenium [122]. To confirm this interpretation, we performed an additional experiment under the same conditions on a film composed of PTBSe blended with the triplet sensitizer PdOEP. The basic idea is that triplets are created very efficiently in the sensitizer and are transfered to the material under study via energy transfer, increasing the population of triplet states [123]. This method was pioneered by Terenin and Ermolaev to measure phosphorescence in organic solutions [124].

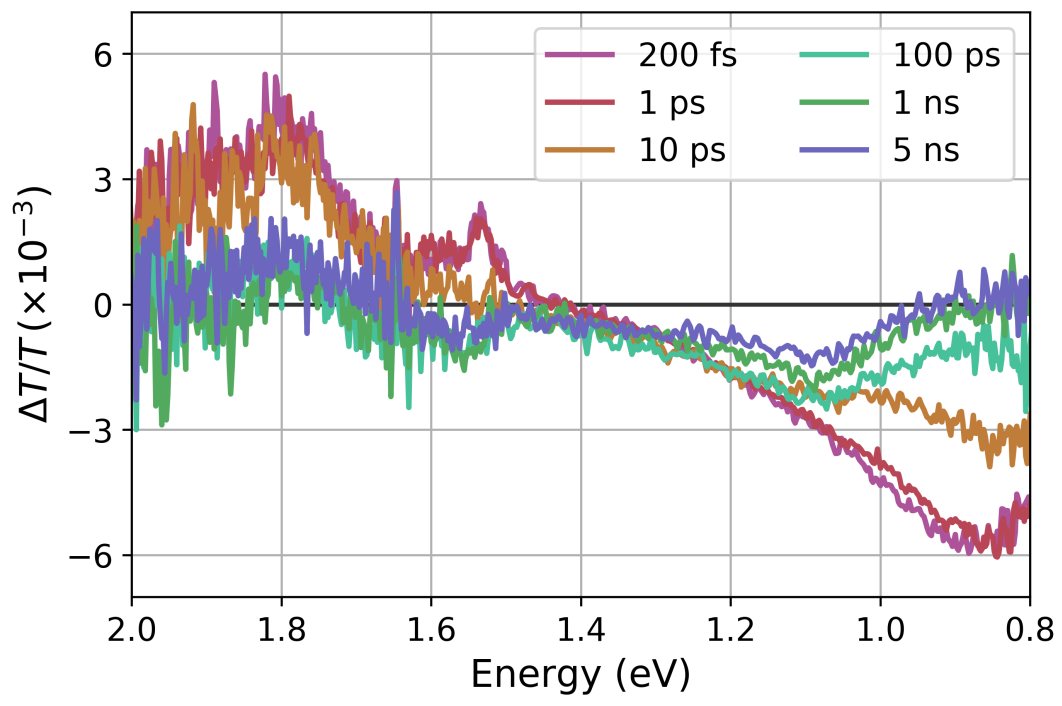

Figure 4.7: Spectral traces of the TAS data measured in a PTBSe neat film at indicated time delays.

Figure 4.8 shows spectra and kinetic profiles obtained for each transient species in PTBSe via the MCR-ALS algorithm, along with the spectra of the sensitized triplets measured in a PTBSe:PdOEP blend film. The first component obtained from the MCR decomposition (component 1 in the figure) is promptly 
created upon light absorption, and is attributed to a singlet exciton PIA. The following component (component 2 in the figure) overlaps reasonably well with the spectra in a PTBSe:PdOEP film and the appearance of this species does not involve characteristic features that would suggest the presence of charged species, such as an electro-absorption feature [53]. Thus, the $\mathrm{PIA}_{2}$ peak is assigned as a triplet exciton PIA. The dynamics of the species in Figure $4.8 \mathrm{~B}$ shows that triplets are formed from singlet excitons via ISC within a rapid time scale of tens of picoseconds and that triplet excitons experience partial decay on the nanosecond time scale. Rapid conversion of primary photo-excitations into the triplet manifold has been previously measured in Seleno- and Telluro- Analogues of Poly(3-hexylthiophene), in Reference [125]. The authors explained that such rapid ISC time scale, along with a decrease in the fluorescence quantum efficiency, was consistent with intersystem crossing facilitated by the heavier selenium and tellurium atoms. 

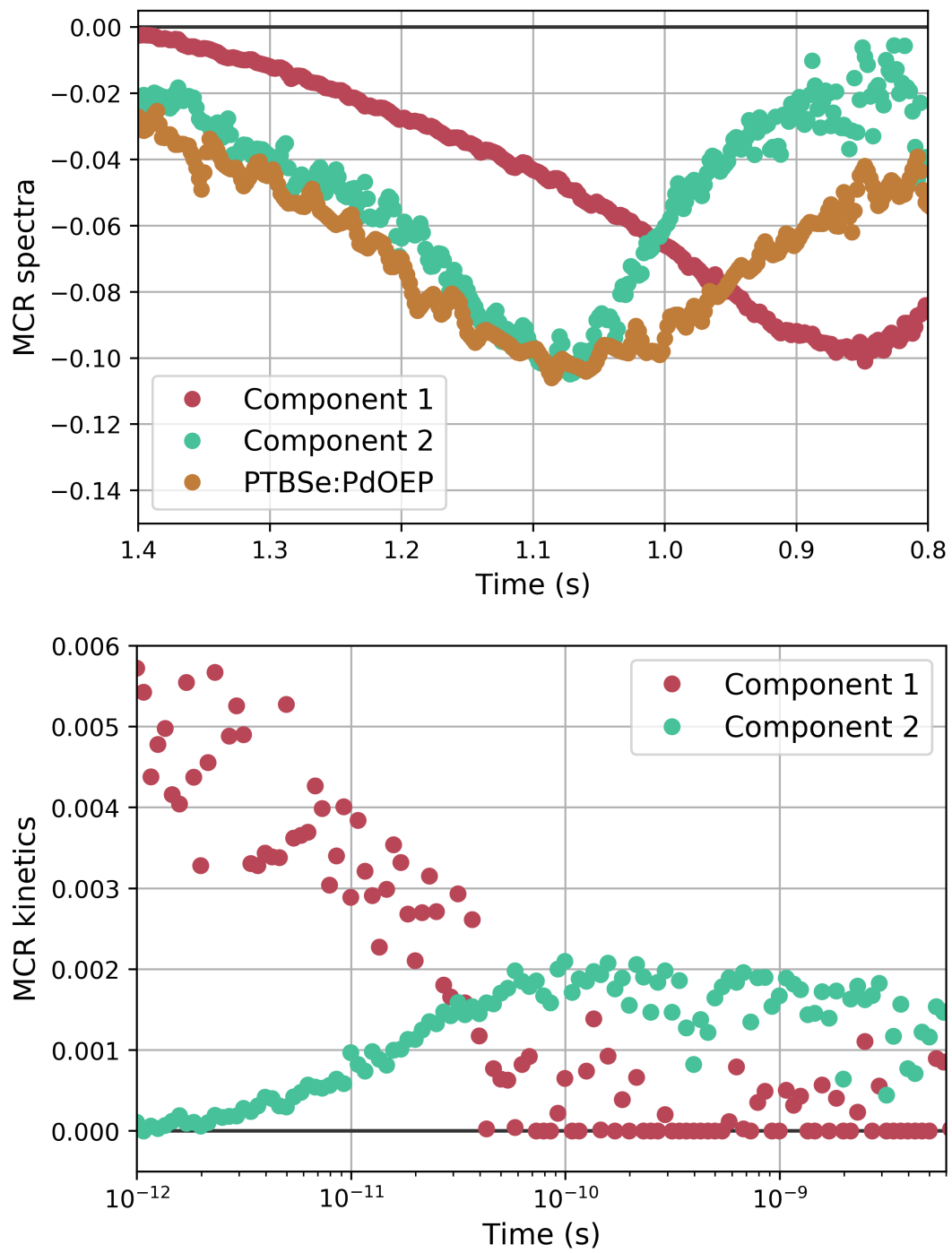

Figure 4.8: A) MCR spectra and B) MCR kinetics for the transient species in a neat fillm of PTBSe. Spectrum of sensitized triplets measured in a PTBSe:PdOEP blend film is also included.

\section{PTBSe:PC 61 BM}

Spectral traces in a blend film of PTBSe:PC ${ }_{61} \mathrm{BM}$ are shown at different time delays in Figure 4.9. First, we note the absence of the singlet exciton feature measured in neat PTBSe, which peaks in the wavelength region $0.8-0.9 \mathrm{eV}$ as shown 
in Figure 4.8 A. This implies that singlet excitons in the blend are quenched in the time scale below the first $200 \mathrm{fs}$, time at which another PIA is measured with a peak value at $1.2 \mathrm{eV}$. Such PIA feature does not match the spectra of triplet excitons shown in Figure $4.8 \mathrm{~A}$, suggesting that it is associated with charge-induced absorption. This ultrafast charge generation upon exciton quenching suggests that donor and acceptor phases are highly intermixed, similarly to the PTBT system, which is consistent with the broad and unstructured steady-state absorption spectra measured in this blend, suggesting the predominance of $\mathrm{H}$-type aggregates due to interchain Coulombic interactions. Interestingly, the spectra evolves over time approaching the long time spectra in neat PTBSe attributed to triplet excitons. As discussed earlier, strong spin-orbit coupling due to the heavy atom effect, facilitates the formation of triplet states. Additionally, in systems where the triplet exciton is lower in energy than the CT states, the decay of CT states into the triplet manifold ${ }^{3} \mathrm{CT} \rightarrow^{1} \mathrm{~T}$ is energetically favorable and represents a loss channel, preventing bound pairs to re-dissociate from CT states into separated carriers. With this in mind, a reasonable interpretation of our results is that bound CT states with triplet character created at the donor:acceptor interfase, decay to the polymer triplet excited state. Population of the ${ }^{3} C T$ state followed by triplet state formation and annihilation was reported by Gehrig et al. in a in PBDTTT-C:PC ${ }_{60} \mathrm{BM}$ system [126]. In agreement with our results, the authors distinguished a progressive red-shift of the TAS photo-induced absorption peak on the sub-nanosecond time scale, due to the formation of triplet excitons. 


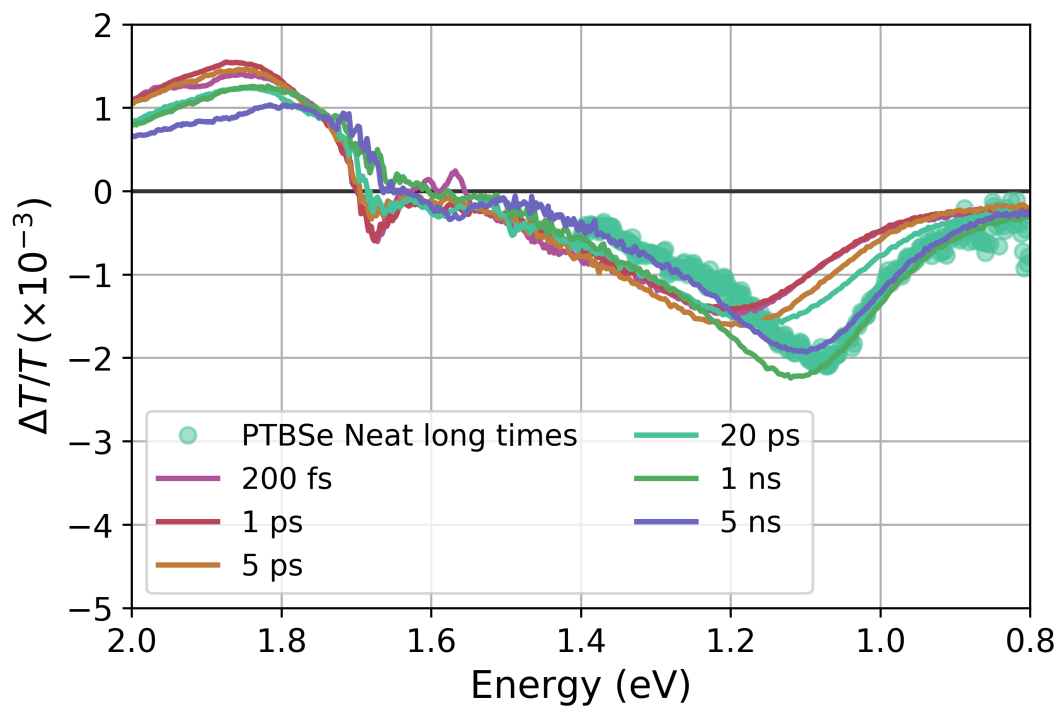

Figure 4.9: Spectral traces in a blend film of PTBSe: $\mathrm{PC}_{61} \mathrm{BM}$ at different time delays along with the spectra in PTBSe neat at long times, assigned to triplet excitons.

The contributions from charges and triplet excitons to the overall TAS signal can be recovered via the MCR-ALS algorithm, using the spectrum at long time delays, where only one component is present, as a spectral mask. In Figure 4.10 we show the temporal profiles obtained, at two different pump fluences. Figure 4.10 A shows that the rate of charge decay does not depend upon pump fluence, suggesting that charges decay into the triplet state via geminate recombination. 
A

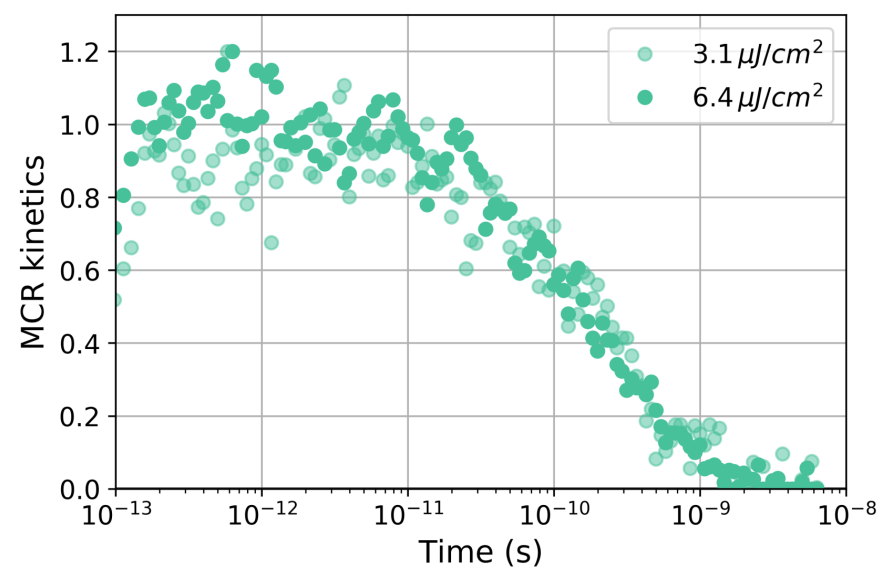

B

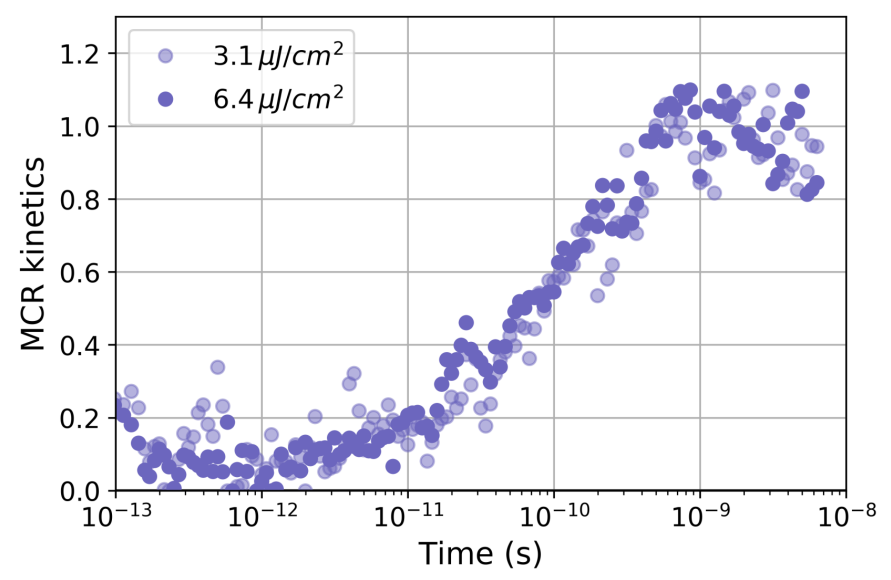

Figure 4.10: $M C R$ kinetics in a blend film of $\mathrm{PTBSe}: \mathrm{PC}_{61} \mathrm{BM}$ at different pump fluences. A) Component 1 , assigned to charges, and B) component 2, assigned to triplet excitons in the polymer.

As explained in Chapter 3, at particular wavelengths we can obtain monochromatic pump pulses by using the harmonic modules of a picosecond laser (fundamental wavelength $1064 \mathrm{~nm}$ ) which in our setup can be electronically delayed with respect to the probe beam resulting in time delays up to $3 \mu \mathrm{s}$. We used this configuration to extend our temporal range on PTBSe:PC ${ }_{61} \mathrm{BM}$ 
using $532 \mathrm{~nm}$ excitation pulses. The collected data matrix exhibits a single PIA feature which is identical to the PIA attributed to triplets in the nanosecond time scale. Figure 4.11 shows the integrated kinetics of triplet states at three different fluences. We observe a relatively short half-life of triplets of $\sim 70 \mathrm{~ns}$, which is essentially independent of the pump fluence. The triplet state can be depopulated by decay to the ground state with an intrinsic monomolecular triplet lifetime that is typically above the nanosecond time scale [127]. However, the triplet state can also decay by transfering its energy to triplet molecular oxygen $\left({ }^{3} \mathrm{O}_{2}\right)$ in the surroundings to generate singlet oxygen $\left({ }^{1} \mathrm{O}_{2}\right)$ [128]. Even though our measurements were performed under dynamic vacuum conditions, minimal amounts of oxygen may have served as a triplet quencher. It is worth noting that formation of highly reactive singlet molecular oxygen should be avoided, since it is expected to react with the polymer backbone creating carbonyl type defects eliminating the conjugation [129].

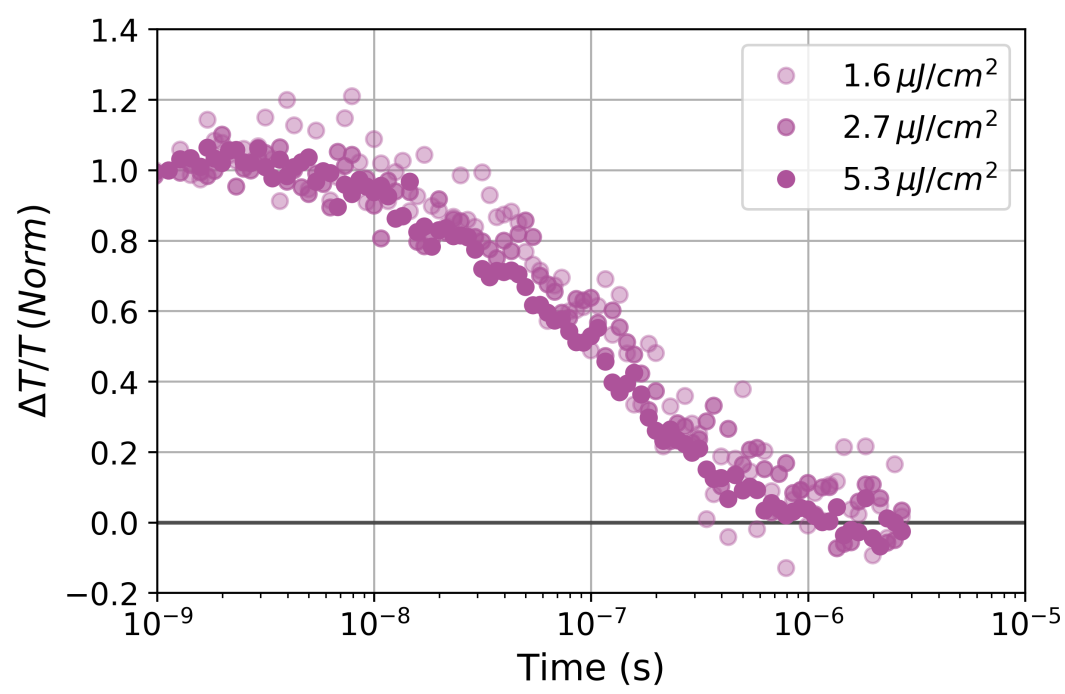

Figure 4.11: Integrated kinetics of triplets in PTBSe:PC ${ }_{61} \mathrm{BM}$ at three different fluences.

The main loss pathway that we have identified in the PTBSe:PC ${ }_{61} \mathrm{BM}$ is 
illustrated in the state diagram in Figure $4.12 \mathrm{C}$, along with the state diagrams of the PTBO system studied here and of the original PTBT system. Dominant pathways are represented with bold colours, and less efficient pathways in dashed colours.
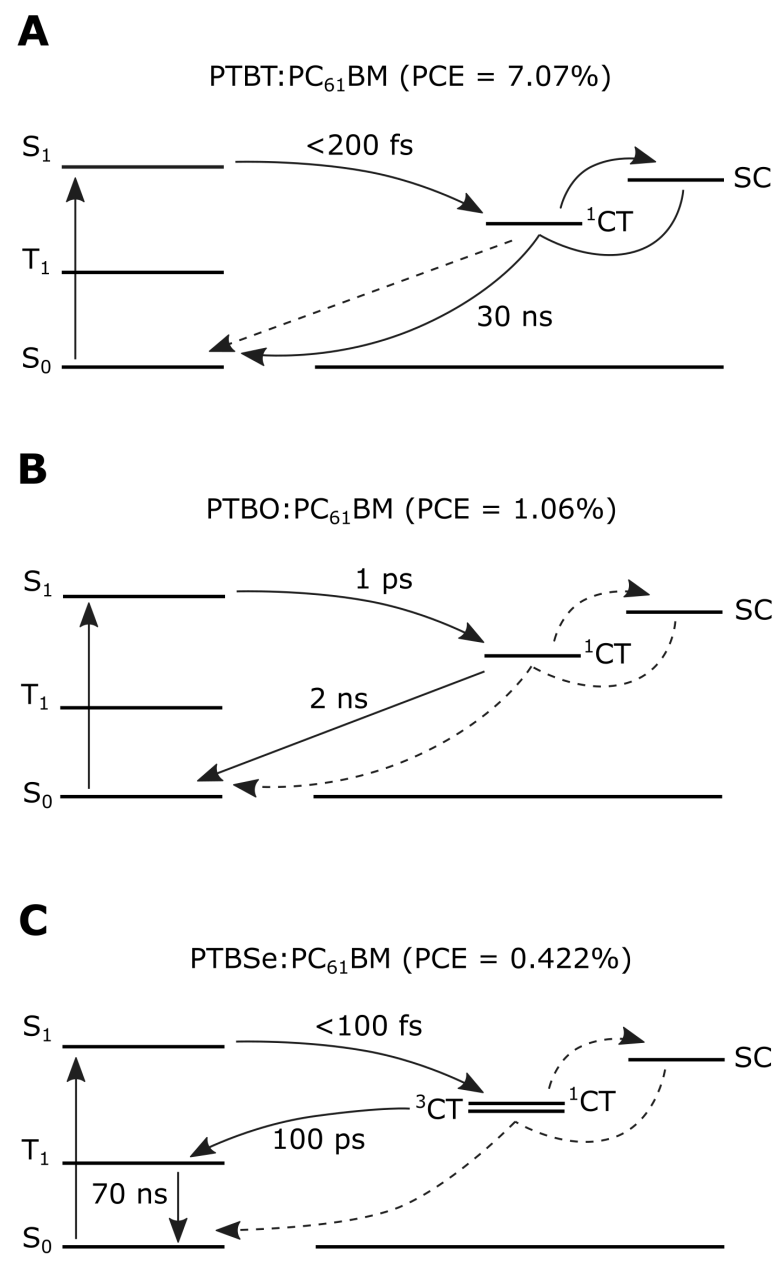

Figure 4.12: State diagrams illustrated for the parent blend PTBT:PC ${ }_{61} \mathrm{BM}(\mathrm{A})$ and the blend films under study in this work, i.e. PTBO:PC ${ }_{61} \mathrm{BM}(\mathrm{B})$ and $\mathrm{PTBSe}_{\mathrm{PC}} \mathrm{C}_{61} \mathrm{BM}(\mathrm{C})$. Dominant pathways are represented with bold colours, and less efficient pathways in dashed colours. 


\subsection{Conclusions}

We have applied ultrafast transient absorption spectroscopy (TAS) to explore the photophysics of a family of polymers derived from the low-band-gap alternating copolymer PTBT where the sulphur atom in the thiadiazole unit was substituted with oxygen or selenium. Overall, our data explains the superior photovoltaic performance of the original PTBT polymer and highlights the low tolerance of these materials to backbone substitutions.

Our TAS measurements on the neat polymers and the polymer:fullerene blends explain that the inferior photovoltaic performance of PTBO and PTBSe with respect to the original PTBT polymer, is caused by different geminate recombination pathways. In PTBO:PC ${ }_{61} \mathrm{BM}$, steady state $\mathrm{UV}$-Vis absorption suggests a predominant contribution from J-aggregates due to intrachain interactions, similar to the PTBT system. Our TAS measurements demonstrate that charges are photo-generated via excitons, with a fraction of $33 \%$ of excitons being promptly quenched within the first $200 \mathrm{fs}$ of our measurements, forming bound charge pairs. The individual contributions from excitons and charges to the overall TA signal were obtained via the soft-modelling algorithm known as Multivariate Curve Resolution by Alternating Least Squares (MCR-ALS) and the population of charges exhibits a strong decay in the sub-nanosecond time scale which does not depend upon excitation fluence. Thus, we conclude that geminate recombination, i.e. recombination of geminate pairs at the donor:acceptor interfase subsequent to the exciton quenching process, is the major limiting factor in this blend system. In PTBSe:PC ${ }_{61} \mathrm{BM}$, however, UV-Vis absorption suggests a predominant contribution from H-type aggregates due to interchain Coulombic interactions. Our TAS measurements show that all excitons are quenched in an ultrafast time scale, suggesting a highly intermixed morphology, leading to a 
charge-induced absorption feature assigned to hole polarons in the polymer. The charge-induced PIA rapidly exhibits a progressive red-shift towards a new absorption peak which is consistent with the spectrum of triplet excitons. Additionally, the rate of charge decay does not depend upon the excitation fluence. In this way, the low photovoltaic performance in the PTBSe variant is attributed to geminate recombination of CT states with triplet character into triplet excitons in the polymer.

Our results are consistent with the work of Ji-Seon Kim et al. in Reference [102], where it was shown, for the closely related polymers PSBS and PSBSe blended with $\mathrm{PC}_{71} \mathrm{BM}$, that the larger selenium heteroatom is unfavourable for close intermolecular packing in the thin film, resulting in a poor blend film morphology and low charge carrier mobility. We thus speculate that unfavorable packing of the $\mathrm{PTBO}$ and PTBSe polymers at the interfaces with $\mathrm{PC}_{61} \mathrm{BM}$ results in suppressed dissociation of CT states and concomitantly, high geminate recombination rates for these systems. Additionally, in the case of PTBSe:PC ${ }_{61} \mathrm{BM}$ the heavy atom effect facilitates the formation of triplet excitons, which is identified as a major loss channel. 


\section{Chapter 5}

\section{Comparative transient absorption}

spectroscopy of fullerene and

\section{non-fullerene organic photovoltaic} cells

\subsection{Overview}

Due to the incorporation of fullerene and its derivatives as electron accepting materials together with the invention of the bulk heterojunction device architecture, organic solar cells demonstrated a jump in efficiency by orders of magnitude [31] [32], compared to previous devices composed by simply neat organic semiconducting materials. For almost two decades, such configurations remained largely unchanged, with active layers composed of low-band-gap polymers in the donor phase and fullerene in the acceptor phase, validated by a continuous growth in PCE. However, the efficiency of fullerene devices was reaching a plateau and furthermore fullerenes are known to be expensive to 
purify, have limited tunability and low absorption in the VIS and NIR regions of the solar spectrum. Additionally, fullerene materials are morphologically unstable in ambient conditions [130].

Due to the limitations of fullerene materials, intense research efforts have been focused on the design and synthesis of nonfullerene acceptors (NFAs), particularly nonfullerene small molecules and oligomers. NFAs are today viable, tunable and low cost acceptors for solution-processed OPVs and some of them have exceded their fullerene counterparts exhibiting the highest certified PCEs for OPVs so far. The highest PCEs to date is of $18.22 \%$ in a single junction device in a laboratory scale [131].

In this chapter we report spectroscopic comparisons of charge generation and recombination in a series of blends featuring the same semicrystalline donor copolymer, PPDT2FBT, matched with different acceptors: the fullerene derivative $\mathrm{PC}_{70} \mathrm{BM}$, the naphthalenediimide-bithiophene copolymer $\mathrm{P}(\mathrm{NDI} 2 \mathrm{OD}-\mathrm{T} 2)$, which is commercially known as N2200, and a small molecular nonfullerene acceptor, NIDCS-HO. These blends were previously found to have power conversion efficiencies of $9.4 \%, 5.0 \%$ and $7.6 \%$, respectively. Using broadband transient absorption spectroscopy from hundreds of femtoseconds to the microsecond timescales with pump wavelengths that selectively excite the donor and the acceptor materials, we investigate the impact of complementary absorption of donor and acceptor and using a simple kinetic model we retrieve quantitative information of the dissociation and recombination processes.

Our comparative results show that in the fullerene and NIDCS-HO -based devices hole transfer from acceptor to donor give rise to a delayed increase of charges in the donor phase. However, the advantage of this contribution to charges that could potentially be extracted in a functional device, is limited 
by the morphology in the NIDCS-HO -based device. Our analysis on charge dissociation and recombination shows that PPDT2FBT:NIDCS-HO benefits from (i) the longest CT lifetime, which is consistent with the smaller energy driving force of this blend, and (ii) the lowest bimolecular recombination rate, on the order of magnitude that has been recommended for efficient NFA devices in recent articles. For this blend, we obtain a recombination order higher than two, which might suggest the contribution of trap assisted recombination in addition to bimolecular recombination between free charges. By simulating the kinetics in absence of disorder, i.e. when the recombination process is strictly bimolecular, we show that charges would live longer before recombining, increasing their chance of being extracted in an operating device. Thus, we speculate that disorder, in addition to the morphology issue, is the main limiting factor in this system. For the all-polymer device based on the N2200 acceptor, we show that both geminate and nongeminate recombination are limiting the photovoltaic performance. The key implication of our results resides in the potential of small molecule NFA acceptors, since morphology and disorder, the factors that we have found to be limiting the device performance, could potentially be tuned for the development of more efficient materials.

\subsection{Background}

\subsubsection{The donor material PPDT2FBT}

PPDT2FBT is a semicrystalline low-bandgap photovoltaic copolymer which was first studied in fullerene devices, demonstrating a well-distributed nanofibrillar networked morphology and balanced hole and electron mobilities (molecular structure is shown in Figure 5.1). This was attributed to its molecular structure where intramolecular and/or intermolecular noncovalent hydrogen 
bonds and dipole-dipole interactions assure strong interchain interactions without losing solution processability [45]. PPDT2FBT:PCBM devices without any post-treatment (annealing, solvent additive, etc.) achieved power conversion efficiencies (PCEs) of over 7\%, and PCE of $9.4 \%$ was proved in a $290 \mathrm{~nm}$ thick conventional single-cell device structure using diphenyl ether (DPE) as an additive and optimal annealing conditions. Furthermore, a high long-term thermal stability of $200 \mathrm{~h}$ at $130^{\circ} \mathrm{C}$ was proved. Because of its high carrier mobility with thickness tolerance, PPDT2FBT has been used as a hole transporting material in methylammonium lead triiodide-based perovskite solar cells (PVSCs) [132].

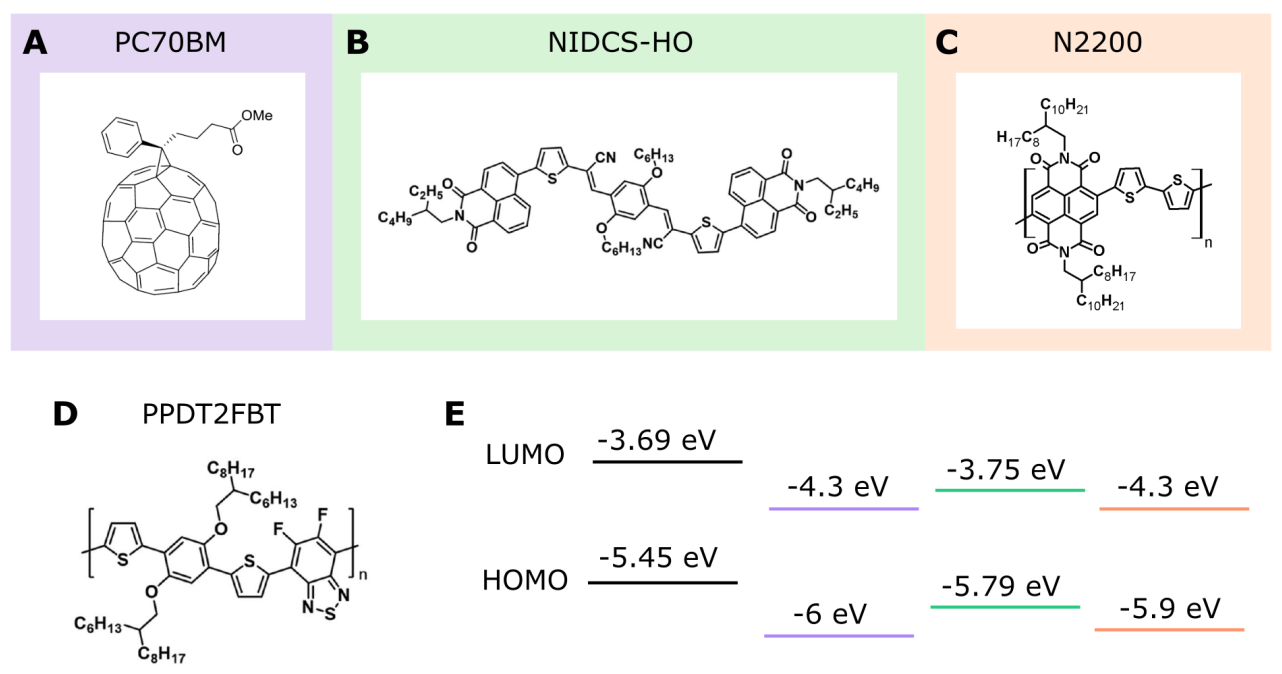

Figure 5.1: Chemical structures of $\mathrm{A}$ ) the fullerene acceptor $\mathrm{PC}_{70} \mathrm{BM}, \mathrm{B}$ ) the nonfullerene small molecule acceptor NIDCS-HO, C) the polymeric acceptor N2200 and D) the semicrystalline low-bandgap copolymer PPDT2FBT. E) Energy level diagrams of the different materials. The HOMO levels were measured by cyclic voltammetry (CV) and the LUMO levels were estimated from the HOMO values and the optical band gaps of the films [45-48]. 


\subsubsection{The small molecule electron acceptor NIDCS-HO}

Molecular acceptors based on a dicyanodistyrylbenzene moiety were known to exhibit efficient electron transporting properties in optoelectronic devices, however their strong self-assembly was resulting in extended domains that would limit their use in bulk heterojuction OPVs. To overcome this defficiency, Kwon et al. incorporated a bulky naphthalimide moiety at the terminal position of the molecule giving rise to the so called DCS-NI acceptors (NIDCS, NIDCS-MO, and NIDCS-HO) which have shown efficient electron accepting and transporting properties, in principle using regioregular poly(3-hexylthiophene) (P3HT) as the donor material [47]. Chemical structure of NIDCS-HO is shown in Figure 5.1. The device performance was tuned by applying a thermal annealing treatment, resulting in an optimised PCE of $2.71 \%$.

Subsequent to its use in combination with P3HT, NIDCS-HO was matched with the donor copolymer PPDT2FBT and optimised devices have shown a maximum PCE of $7.6 \%$ with $V_{O C}=1.03 \mathrm{~V}$ after optimal annealing treatment. The authors demonstrated that thermal annealing favored a well-organized crystalline structure of the NIDCS-HO acceptor without disrupting the faceon packing behavior of PPDT2FBT. However, the short-circuit current and the fill factor were lower than in PPDT2FBT:PCBM devices and the highest PCE achieved was of $7.6 \%$ [47].

\subsubsection{The polymer electron acceptor $\mathrm{N} 2200$}

The development of electron accepting polymers, i.e. polymers with electron withdrawing functional groups and high electron mobility, such as perylene diimide (PDI) and naphthalene diimide (NDI) -based copolymers, have permitted the enhancement of device performance in all-polymer OPVs. However, 
their PCEs are still lower than in polymer:fullerene and polymer:small molecule devices, mainly due to their low $J_{s c}$ and FF.

The most widely used polymer acceptor today is a NDI-based polymer named P(NDI2OD-T2) and commercially known as N2200 (chemical structure in Figure 5.1). This very efficient polymer acceptor was first reported for use in organic thin film transistors [133], showing a high electron mobility of $0.45-0.85 \mathrm{~cm}^{2} /(\mathrm{Vs})$ under ambient conditions in combination with gold contacts and various polymeric dielectrics. Due to its high bulk electron mobility, good solubility in commonly used solvents, near-infrared absorption and wellaligned energy levels, N2200 was a promising candidate for all-polymer OPVs.

N2200 was first used in organic photovoltaic devices by Moore et al. in 2011 [134]. As mentioned above, due to the high mobility in field effect transistors (FETs), this material was a promising candidate for organic solar cells. However, PCEs below $0.2 \%$ were reported for a P3HT:P(ND12OD-T2) device in this early work. To understand this issue, the authors performed TAS measurements on the neat materials P3HT and P(ND12OD-T2) and on the P3HT:P(ND12OD-T2) blend using $495 \mathrm{~nm}$ pump pulses. Transient spectra of the neat materials and the blend are shown at a time delay of 3 ps in Figure 5.2. The TA spectrum of the blend (blue solid line) shows a weak positive signal around $700 \mathrm{~nm}$, the same region where a positive SE is observed in P3HT and a positive GSB is observed in $\mathrm{P}(\mathrm{ND} 12 \mathrm{OD}-$ T2), and a broad PIA at $750-950 \mathrm{~nm}$. Because of the higher absorption cross section of P3HT at $495 \mathrm{~nm}, \mathrm{P} 3 \mathrm{HT}$ is predominantly excited in the blend. The negative PIA at the spectral region $750-820 \mathrm{~nm}$ in the blend is not present in the neat films (in the neat films the TA signal is positive in such spectral region) and the authors attributed it to charges formed on sub-picosecond timescales, overlaping with the exciton signal. At longer time delays, a shoulder in the PIA 
band is observed at $800-850 \mathrm{~nm}$, which is consistent with the polaron band of $\mathrm{P}(\mathrm{NDI}$ 2OD-T2) previously measured by charge modulation spectroscopy on $\mathrm{P}(\mathrm{ND} 12 \mathrm{OD}-\mathrm{T} 2)$ transistors. Therefore, the authors explain, it is likely that the P3HT and P(ND12OD-T2) polaron bands overlap and charges on both polymers are measured. Despite fast charge formation in the first $20 \mathrm{ps}$, the overall charge signal decreases over the first 200 ps. At $1 \mathrm{~ns}$ after excitation the PIA intensity has been reduced to $15 \%$ of its initial value, whereas the remaining charges are longlived and decay over time-scales of hundreds of nanoseconds to microseconds. The authors claim that the blend suffers from fast geminate recombination and attribute this to the specific electronic and conformational properties of the P3HT and P(ND12OD-T2) molecular interface and the nanoscale morphology.

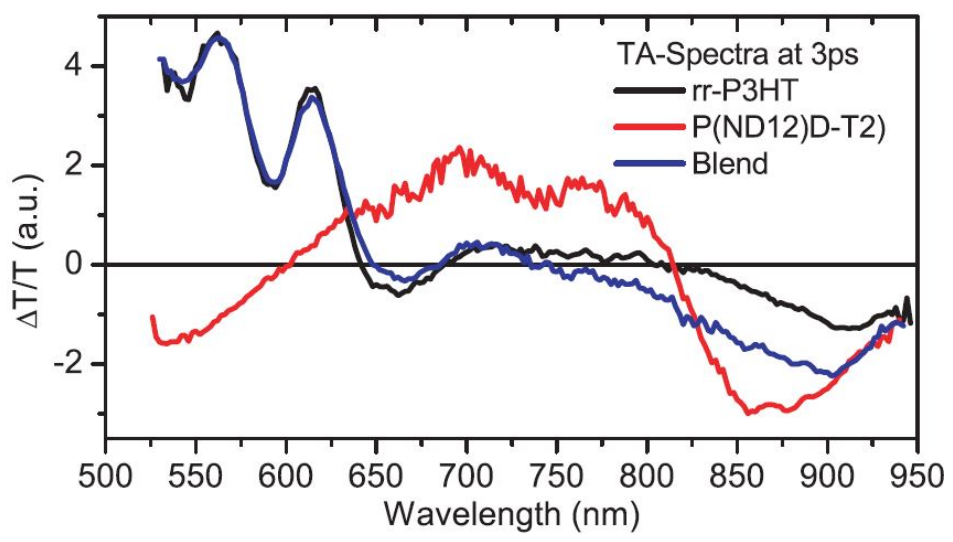

Figure 5.2: $\mathrm{TA}$ spectra of neat $\mathrm{P} 3 \mathrm{HT}$ and $\mathrm{P}(\mathrm{ND} 12 \mathrm{OD}-\mathrm{T} 2)$ (commercially known as N2200) films and of a P3HT:P(NDI2OD-T2) blend 3 ps after excitation. The Figure is reproduced with permission from Reference [134].

In another study, a comparison of the device physics, dynamics and nanoscale film morphology of PTP8:PC71BM (with PCE = 6.0\%) and PTP8:P(NDI2ODT2) (with PCE $=4.1 \%$ ) was performed by Yuan et al. [135]. The researchers show that photoluminescence (PL) signals in both systems are quenched efficiently 
and therefore exciton diffusion to the donor:acceptor interface and subsequent charge transfer are efficient in both systems. They performed TAS measurements on neat and blend films using $400 \mathrm{~nm}$ pump pulses, showing that excitons originate from both PTP8 and P(NDI2OD-T2) and they recognised signal contributions from both components in the blend. In order to quantify geminate recombination in the nonfullerene blend, the authors used the GSB signal, but contributions to the GSB needed to be isolated. They estimated the P(NDI2ODT2) contribution to the GSB from the signal at $750 \mathrm{~nm}$ where the transient signal arises only from $\mathrm{P}(\mathrm{NDI} 2 \mathrm{OD}-\mathrm{T} 2)$, because of negligible transient signal in the neat PTP8 film in such region. In this way, they estimated a 15\% spectral weight from $\mathrm{P}(\mathrm{NDI} 2 \mathrm{OD}-\mathrm{T} 2)$. After deducting the contribution from $\mathrm{P}(\mathrm{NDI} 2 \mathrm{OD}-\mathrm{T} 2)$, the authors distinguished two regions in the dynamics of the transient GSB signal from PTP8: 1) a rapid decay of $57.2 \%$ within 50 ps which they atribute to geminate recombination of excitons and CT states, and 2) a slow decay after 50 ps due to nongeminate recombination. For the PTP8:PCBM blend, the authors consider the absorption of PCBM as negligible and that therefore the GSB consists of only the PTP8 contribution. The GSB decay suggest a fraction of geminate recombination of only $6.7 \%$, in contrast with the $57.2 \%$ they found in the $\mathrm{P}(\mathrm{NDI} 2 \mathrm{OD}-\mathrm{T} 2)$ blend. The recombination of charges at longer time delays, of the order of hundreds of ps, is similar for the PTP8:P(NDI2OD-T2) and PTP8:PC71BM blends. Therefore, the better carrier generation in PTP8:PC71BM is considered by the authors the prominent factor for the higher EQE of the fullerene blend. Their morphology analysis shows that the low charge generation efficiency in PTP8:P(NDI2OD-T2) can be due to reduced crystallinity of $\mathrm{P}(\mathrm{NDI} 2 \mathrm{OD}-\mathrm{T} 2)$ in the blend film and unfavorable face-to-edge orientation at the donor acceptor interface.

A study for understanding the effect of annealing in a P(NDI2OD-T2) blend 
was perfomed by Karuthedath et al. in 2018 [62]. With a combination of steady state and time resolved spectroscopic techniques in TQ1:P(NDI2OD-T2) devices, the researchers concluded that the increase of PCE from $2 \%$ to $3.15 \%$ after thermal annealing $\left(100^{\circ} \mathrm{C}\right.$ for $\left.15 \mathrm{~min}\right)$ is due to the reduction of geminate recombination, but that nongeminate recombination is a significant efficiency loss process even in annealed TQ1:P(NDI2OD-T2) devices. The authors extracted spectra and kinetics of unbound charges from TAS measurements at sufficiently long time delays (nanosecond to microseconds) such that the contribution from excitons is no longer present and kinetics is shown at different excitation fluences for the system with and without annealing. In order to quantify geminate and nongeminate recombination, the authors used the ultrafast charge generation model described in Section 2.9 of this thesis [61], to global fit the data across all measured fluences with shared fitting parameters. The output parameters show that $93 \%$ of the photogenerated carriers in the annealed film are spatially separated and thus could potentially be extracted from the solar cell and an effective bimolecular recombination coefficient of $1.2 \times 10^{-12} \mathrm{~cm}^{3} / \mathrm{s}$ was calculated from the fit parameters using a carrier concentration of $5 \times 10^{15} \mathrm{~cm}^{-3}$ (approximately equivalent to 1 sun illumination). Other output parameters for this film are shown in Table 2.2 of this thesis. In contrast, in the as spun blend $65 \%$ of the photogenerated carriers are spatially separated and the effective bimolecular recombination coefficient is $0.3 \times 10^{-12} \mathrm{~cm}^{3} / \mathrm{s}$. The recombination order $\lambda+1$ is also a free parameter in their model and it is higher than 2 for both blends (2.36 in the annealed blend and 2.53 in the as-spun blend).

A series of PPDT2FBT donor polymers with different molecular weight have been synthesized and investigated in OPV devices using P(NDI2OD-T2) as the acceptor material. With increasing polymer donor molecular weight, the domain size of the PPDT2FBT:P(NDI2OD-T2) blend film was reduced from $225 \mathrm{~nm}$ to 115 
$\mathrm{nm}$ and a superior degree of intermixing was observed by AFM. The optimized morphology led to a PCE of $5.10 \%$ for the high-molecular weight donor [46].

\subsubsection{Fullerene vs. nonfullerene organic photovoltaics}

The power conversion efficiency of organic solar cells continues to grow thanks to the development of high efficiency nonfullerene acceptors and further improvements in PCE are expected in this field. To produce best performing devices in a rational way, it is necessary to uncover relationships between material properties (e.g. carrier mobilities, recombination rates) and photovoltaic parameters and identify potential optimisations.

A recent study based on drift-diffusion simulations has revealed that NFA OPVs can potentially reach PCEs above $18 \%$ with mobility values readily achievable in existing material systems [136]. In their model, enhanced absorption due to the complementary absorption of the donor and acceptor materials leads to a gain in $J_{s c}$, while $V_{O C}$ decreases when using narrower bandgap materials. The authors advise that with the already reported hole and electron mobilities of $5 \times 10^{-4} \mathrm{~cm}^{2} \mathrm{~V}^{-1}$, single-junction NFA OPVs can potentially reach PCEs above $18 \%$ if the nongeminate recombination rate constant is reduced to $1 \times 10^{-12} \mathrm{~cm}^{3} \mathrm{~s}^{-1}$ and IQE increased to $95 \%$.

Free charge generation in a high performance blend of the donor polymer PM6 with the nonfullerene acceptor Y6 was studied recently with experimental and theoretical techniques combined, suggesting that in this system charge generation is assisted by the electrostatic interfacial field which for $\mathrm{Y} 6$ is large enough to compensate the Coulomb dissociation barrier [77]. The authors demonstrate that the efficiency of their devices is mainly limited by insufficient 
photon absorption and free charge extraction. Via drift-diffusion simulations utilising their measurements of mobilities and bimolecular recombination rate, they are able to reproduce the JV curves and show that the efficiency could be further improved with a smaller recombination coefficient and carrier mobility one order of magnitude higher. Interestingly, a device based on the new copolymer donor D18 in a D18:Y6 device recently demonstrated reduced recombination and balanced charge carrier transport, resulting in a PCE above 18\% [131].

In light of the aforementioned studies, we address the comparison between our fullerene and nonfullerene photovoltaic devices by directly monitoring the excited states using transient absorption spectroscopy with pump wavelengths to selectively excite donor and acceptor materials at various pump fluences. We aim to (i) explain the unexpected low value of $J_{s c}$ in the PPDT2FBT:NIDCSHO device, despite of its broad absorption due to complementary absorption of donor and aceptor, and (ii) compare charge generation, dissociation and recombination in these systems to determine their performance limiting factors.

\subsection{Results and discussions}

\subsubsection{Device characteristics}

Table 6.1 summarizes the photovoltaic characteristics in the optimized devices under study, as reported in the literature [45] [46] [47]. We can observe that the fullerene device shows the highest $J_{s c}$ and $F F$, resulting in the highest PCE, whereas the nonfullerene devices exhibit higher $V_{o c}$ but fail in the other photovoltaic parameters. The external quantum efficiency (EQE), defined as the number of extracted electrons with respect to the number of incident photons at short-circuit condition, is higher in the fullerene devices. 
Regarding the HOMO and LUMO energy levels (shown in Figure 5.1), the three donor:acceptor systems exhibit well-matched energy level alignments, with the smallest energy offsets of LUMO-LUMO and HOMO-HOMO in the case of PPDT2FBT:NIDCS-HO. A smaller energy driving force results in a higher CT energy and consequently a suppression of vibronic coupling to the ground state [137] [138], explaining the higher magnitude of $V_{O C}$ in the PPDT2FBT:NIDCS-HO system.

Table 5.1: Photovoltaic characteristics in the optimized devices under study [45] [47] [46].

\begin{tabular}{cccc}
\hline & D:PC $_{\mathbf{7 0}}$ BM [45] & D:NIDCS-HO [47] & D:N2200 [46] \\
\hline$V_{o c}$ & 0.79 & 1.03 & 0.85 \\
\hline$J_{s c}$ & 16.3 & 11.88 & 11.9 \\
\hline FF & 0.73 & 0.63 & 0.51 \\
\hline PCE & 9.4 & 7.6 & 5.1 \\
\hline EQE (532nm) & 0.8 & 0.7 & 0.4 \\
\hline
\end{tabular}

\subsubsection{UV-Vis steady state absorption}

As explained in Section 3.2 , it is a standard practice to measure the steady state UV-Vis absorption spectra of samples before and after carrying out a TAS experiment, to determine their ground state absorption and as a way to verify that no degradation has occurred during the experiment. UV-Vis absorption of the neat donor PPDT2FBT and its fullerene and nonfullerene OPV blend films in Figure 5.3 were obtained in a Cary 50 UV-Vis Spectrophotometer. For every film 
an intense peak is observed at $\lambda \approx 650 \mathrm{~nm}$ and additional peaks are exhibited by the blends, the molecular acceptor blend in particular exhibiting high intensity peaks complementary to the donor material. TAS experiments were performed with excitation wavelengths indicated with vertical lines in the figure.

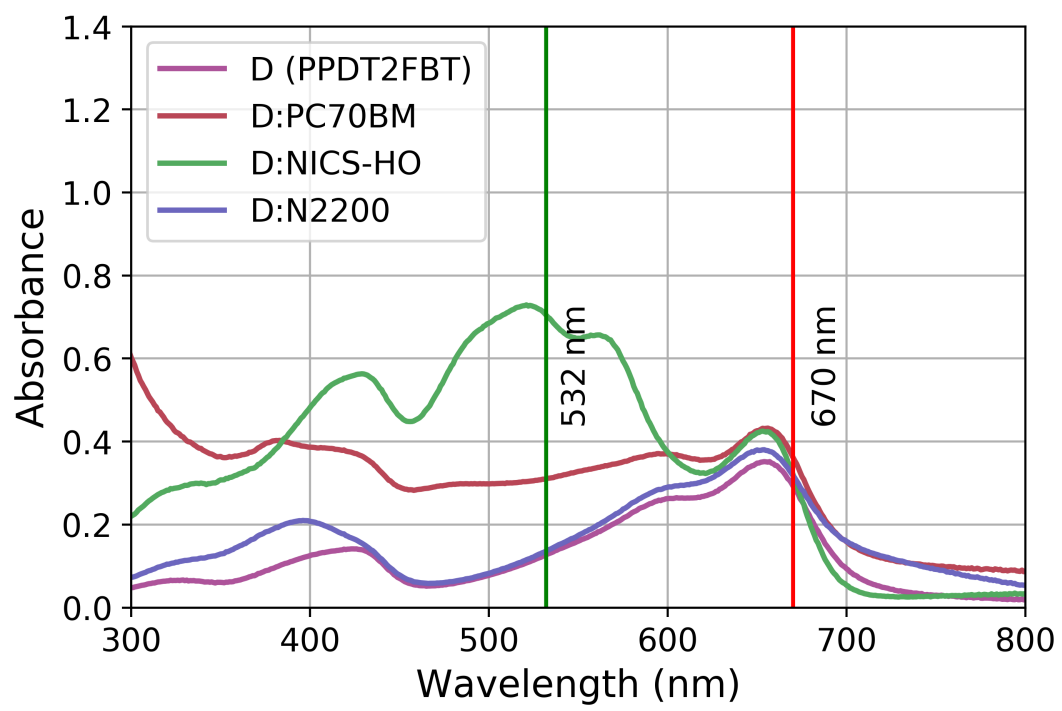

Figure 5.3: UV-Vis absorption spectra of the neat donor PPDT2FBT and the fullerene and nonfullerene OPV blend films.

\subsubsection{Transient absorption spectroscopy}

We measured transient absorption on the neat films and blends using two different excitation wavelengths (532 and $670 \mathrm{~nm}$ ) and various excitation fluences. $532 \mathrm{~nm}$ pump pulses can be obtained by tuning a parametric amplifier (TOPAS) and using the retroreflector mounted in a mechanical delay stage to obtain pump-probe delays up to $6 \mathrm{~ns}$. Additionally, $532 \mathrm{~nm}$ pulses can be obtained by using the second harmonic module of a picosecond laser with fundamental wavelength $1064 \mathrm{~nm}$. This beam is electronically delayed with respect to the probe beam resulting in time delays up to $3 \mu \mathrm{s}$. $670 \mathrm{~nm}$ pulses, however, can only 
be created using the TOPAS and time delays are limited to the nanosecond time scale. More details on the experimental setup are described in Section 3.3 of this thesis.

\subsubsection{Excited states in the neat materials}

Before performing TAS experiments on the donor:acceptor blend films, we performed TAS experiments on films of pristine donor and acceptor materials. This allows us to identify the absorption spectrum of excitons, which assists our interpretation of the spectral features on the blends.

\section{Neat PPDT2FBT}

TA spectral slices are shown in Figure 5.4 A for a pristine film of our donor material PPDT2FBT pumped by $532 \mathrm{~nm}$ pulses of $2.6 \mu \mathrm{J} / \mathrm{cm}^{2}$ fluence. As often encountered in neat materials, factorisation of the data matrix via singular value decomposition (SVD) suggests the presence of a singular excitonic component, created immediately after excitation. The spectral features observed consist of the ground state bleach (GSB) over the spectral region 1.8-2.4 eV, a photoinduced absorption (PIA) over the region $0.8-1.6 \mathrm{eV}$ and weak electro-absorption (EA) around $1.7 \mathrm{eV}$ (A detailed description about these TA features can be found in Chapter 3). The dynamics of the GSB and PIA features were obtained by direct integration over their respective spectral regions, exhibiting decays that follow the same dynamics, as expected for a single component matrix (Figure $5.4 \mathrm{~B})$. However, a single exponential is an insufficient fit for the decay, whereas a fit function consisting of two exponentials with time constants of $12 \mathrm{ps}$ and 126 ps can successfully explain the data. Such biphasic behaviour, with a fast component and a slower component in the TA dynamics of neat materials has been understood as inhomogeneity in the exciton population due to disorder, 
breaking the conjugation of the chromophores into segments of different lengths [139]. The decay of these features is independent of pump fluence, and therefore nonlinear recombination effects such as bimolecular exciton annihilation can be ruled out.
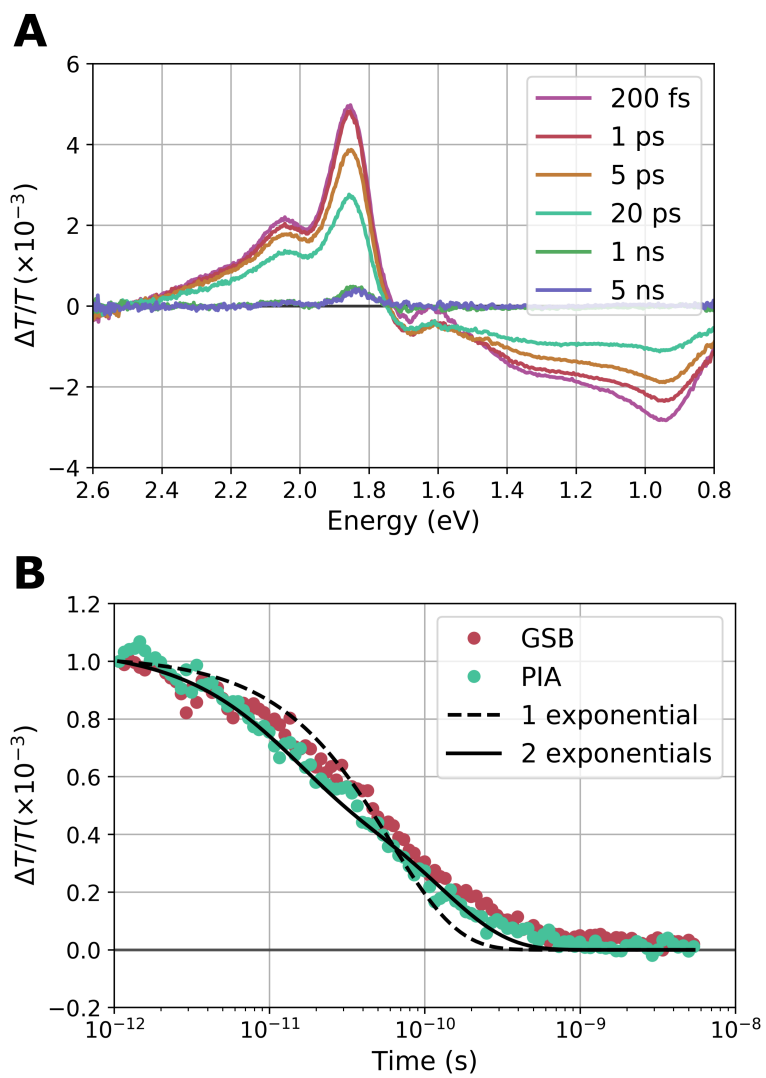

Figure 5.4: A) Low fluence $\left(2.6 \mu \mathrm{J} / \mathrm{cm}^{2}\right)$ TA spectral slices of a neat film of PPDT2FBT at indicated time delays. B) Integrated kinetics of the GSB and PIA features.

\section{Neat NIDCS-HO}

We measured transient absorption of pristine films of NIDCS-HO under the same conditions as the pristine PPDT2FBT film, which exhibited the spectral traces shown in Figure 5.5. In this case we observe spectral evolution of the PIA feature within the first picosecond. In effect, SVD of the data matrix returns 
singular values that suggest the presence of two components. Pure spectra of these two transient components and its associated kinetics were obtained via MCR-ALS bilinear decomposition using the spectra of the second component at long time delays (where the first component is no longer present) as a spectral mask and are presented in figure 5.6. Component 1 decays within the picosecond timescale and component 2 peaks over tens of picoseconds and decays over hundreds of picoseconds. Additionally, component 2 contains an EA feature, suggesting the population of charge (polaron) pairs [140]. It is unlikely that triplet states are present, since triplets would need more time to form via intersystem crossing and also more time to decay [141]. A photophysical study on the related small molecule acceptor NIDCS-MO [142] shows similar features in the picosecond timescale, however the spectral region around $1.6 \mathrm{eV}$ was not accessible with their TAS setup.

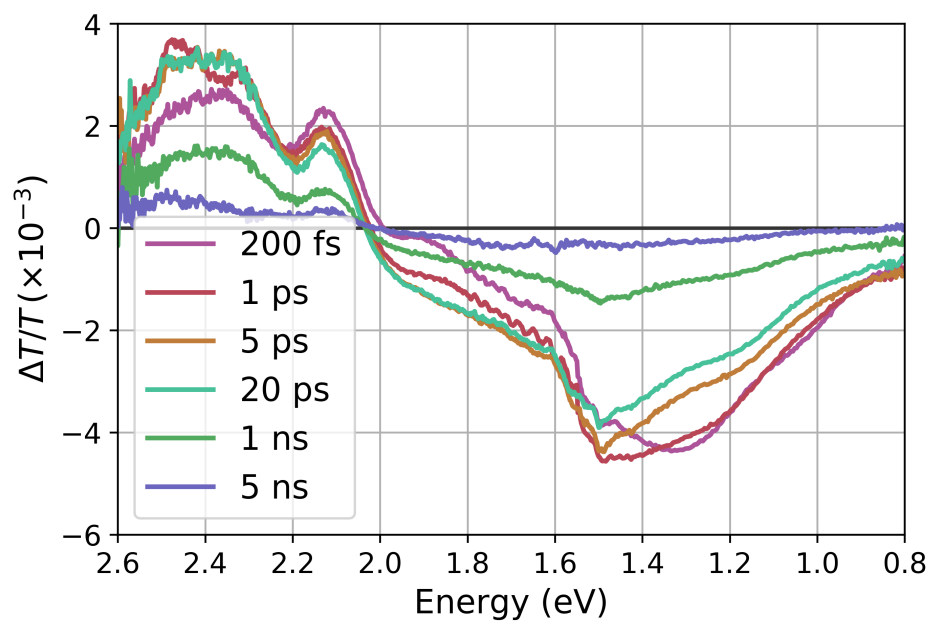

Figure 5.5: TA spectral slices of a neat film of NIDCS-HO at indicated time delays. 

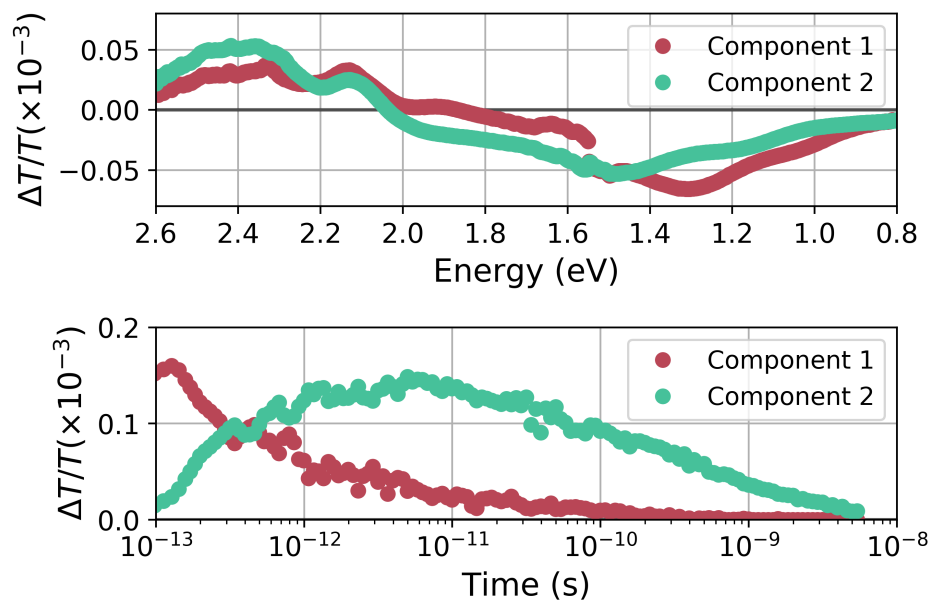

Figure 5.6: A) MCR spectra and B) kinetics of the TAS components in a film of NIDCSHO.

\section{Neat $\mathrm{PC}_{70} \mathrm{BM}$}

Regarding the fullerene acceptor, contributions from the transient PCBM anion are usually overwhelmed by the positive polaron in the donor phase. Reference [143] shows that the PCBM anion has a peak molar absorption coefficient of $6 \times 10^{3} \mathrm{M}^{-1} \mathrm{~cm}^{-1}$ which corresponds to an absorption cross-section of $2.3 \times 10^{-17} \mathrm{~cm}^{2}$. As will be seen below, such cross-section is an order of magnitude lower than our experimental value in the blend. Additionally, the PIA peak measured in our fullerene blend is nearly identical as the one found in the molecular acceptor blend, suggesting that such peak relates to hole polarons in the donor phase [144].

\section{Neat N2200}

Regarding our polymeric acceptor, transient spectra of a N2200 film found in figure 5.2 shows a ground state bleach (GSB) in the region of $600-820 \mathrm{~nm}$ and excited state photo-induced absorption (PIA) bands below and above the GSB. This information will be used to distinguish the N2200 contribution to the 
overall PIA signal of this blend.

\subsubsection{Donor:acceptor blend films - Assignment of the transient spectral features}

Transient species that contribute to the TAS signal at short time delays were desentangled using the soft modelling technique MCR-ALS with known spectra from the neat materials used as constraints. As explained in Chapter 3, this bilinear decomposition of the multi-component data returns the time-independent pure spectra of each transient component and the concentration profiles or kinetics for each component. In this section we focus on the MCR spectra obtained for each blend, and its corresponding kinetics is explored in the following section to analyse the charge generation process.

\section{PPDT2FBT:PC 70 BM}

TA spectral slices of a PPDT2FBT:PCBM film are presented in Figure 5.7 A and B for short and long time delays respectively. For this blend, SVD suggests the presence of 2 transient components for time delays below $6 \mathrm{~ns}$ and only one component left at longer time delays. The spectra of neat donor PPDT2FBT is superimposed in Figure 5.7 A, showing a strong overlap between the exciton in the donor material and the early time signal in the blend. Therefore, component 1 at early time delays is associated with the population of singlet excitons in the donor phase. Spectra of the other component obtained via MCR-ALS is shown in Figure 5.8. As mentioned above, the long time data is composed by only one component which decays in the microsecond time scale, and its spectra has a strong overlap with the spectra of component 2 at early times. In principle, such long-lived species can be due to the formation of triplet states, CT states or polarons. Because of the high EQE of this system and also because the decay 
of this feature depends upon the excitation density (as will be seen below), this component is interpreted as a charge feature due to the population of CT states and charged polarons in the blend.

It is worth noting here that the interpretation of the components obtained via MCR-ALS is known to be subjective and guided by additional knowledge on the particular systems [93]. Throughout this thesis, the determination of the number of components has been also assisted upon experimentation, analysing the results obtained assuming different number of components.
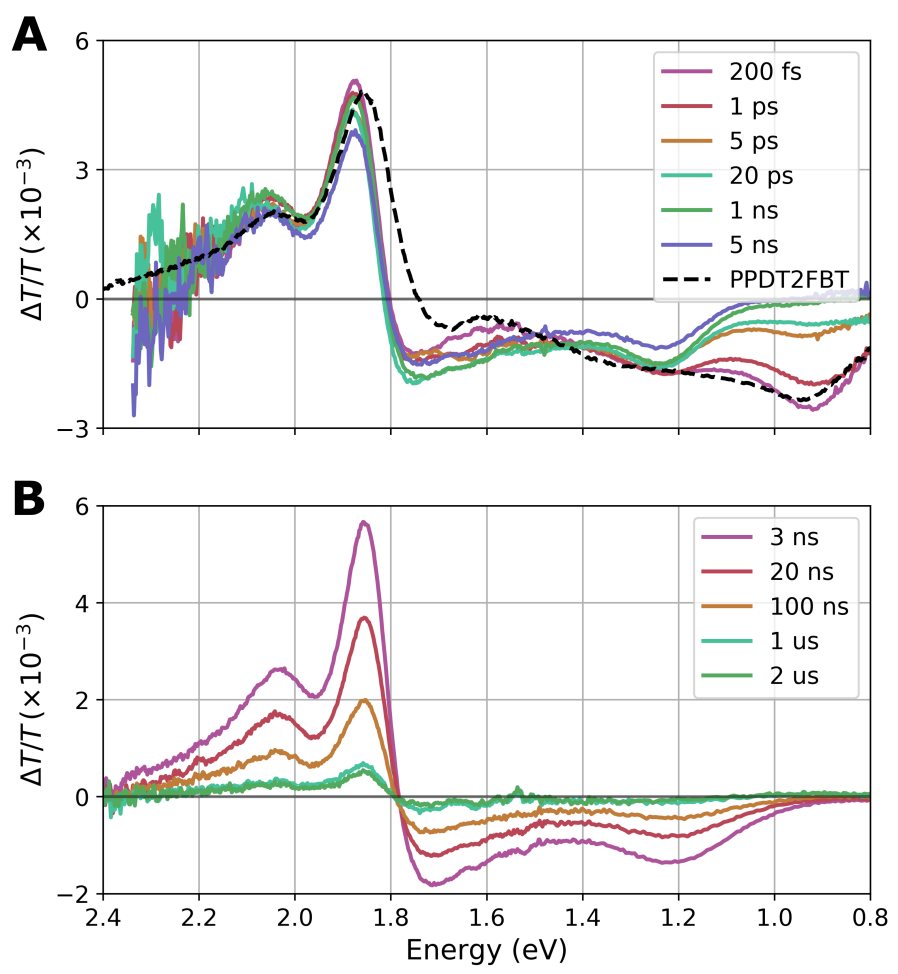

Figure 5.7: TA spectral slices of a film of PPDT2FBT:PCBM at indicated time delays.

We note in figure 5.8 that the MCR charge spectra at early times is nearly identical to the real spectra at long time delays, with small differences in the PIA 
region due to the numerical steps involved in the bilinear decomposition. The charge spectra exhibits a strong negative peak to the red side of the GSB, which is known as electro-absorption (EA) and will be discussed in more detail at the end of this section.

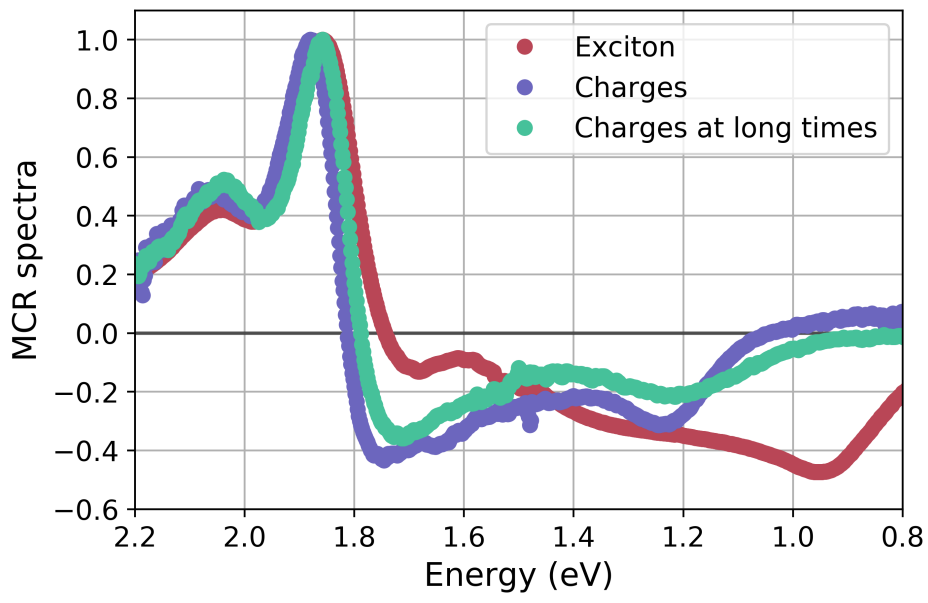

Figure 5.8: MCR spectral components in a film of PPDT2FBT:PCBM, obtained by softmodelling, including the TAS spectra at longer time delays.

\section{PPDT2FBT:NIDCS-HO}

In the case of TAS measurements in a PPDT2FBT:NIDCS-HO blend, $532 \mathrm{~nm}$ excitation pulses can be absorbed not only in the donor material PPDT2FBT, but also (and predominatly) in the molecular acceptor NIDCS-HO (Figure 5.3). Figure 5.9 shows TA spectral traces at short and long time delays and spectra of the exciton features measured in the pristine materials are also included, to show their overlap with the early time spectra of the blend. 

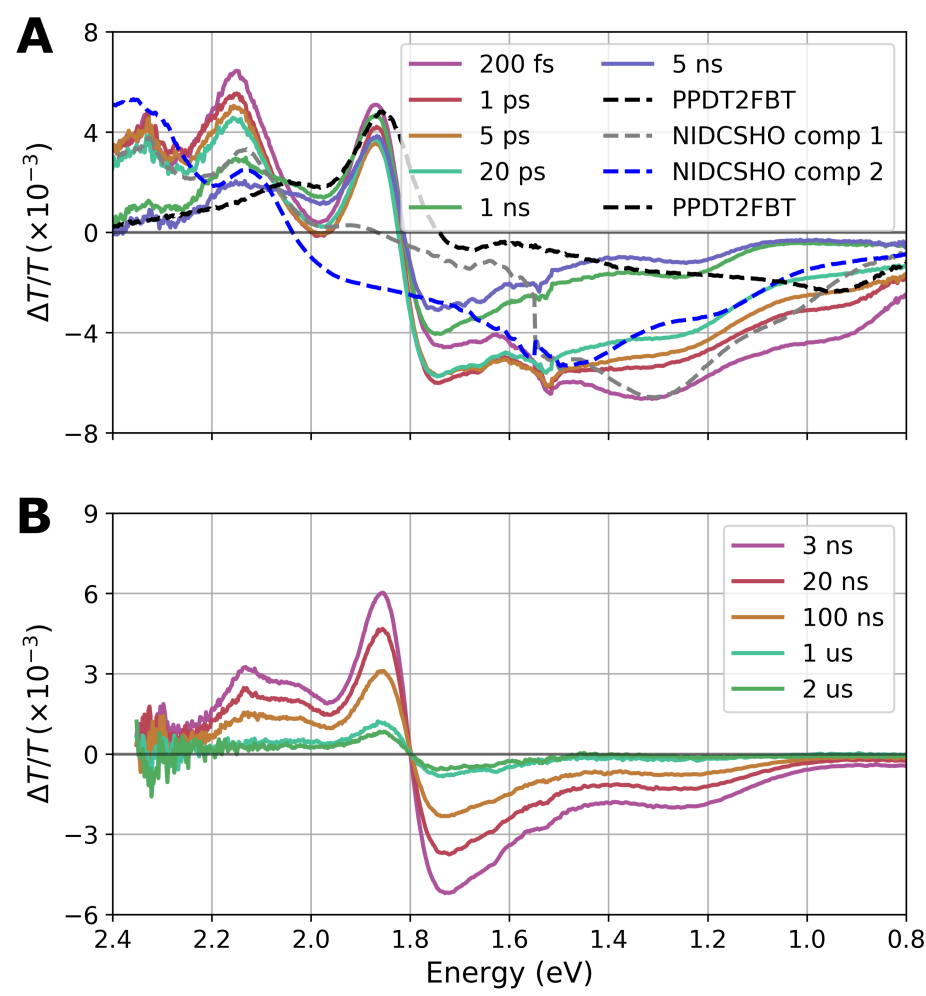

Figure 5.9: TA spectral slices of a film of PPDT2FBT:NIDCS-HO at indicated time delays.

TAS data below 6 ns was deconvoluted via MCR-ALS using the spectra in neat PPDT2FBT and NIDCS-HO (shown in Figure 5.9 A) used as spectral masks, i.e. fixing three components to those spectral profiles. In this way, a fourth component is obtained without rotational ambiguity. Resulting MCR spectra are shown in Figure 5.10. At longer time scales, up to the microseconds, only one component is found via SVD, and Figure 5.10 shows that spectra of such component matches component 4 at early time delays. Under the same criteria as with the fullerene blend, such long lived component is attributed to a charge feature. 


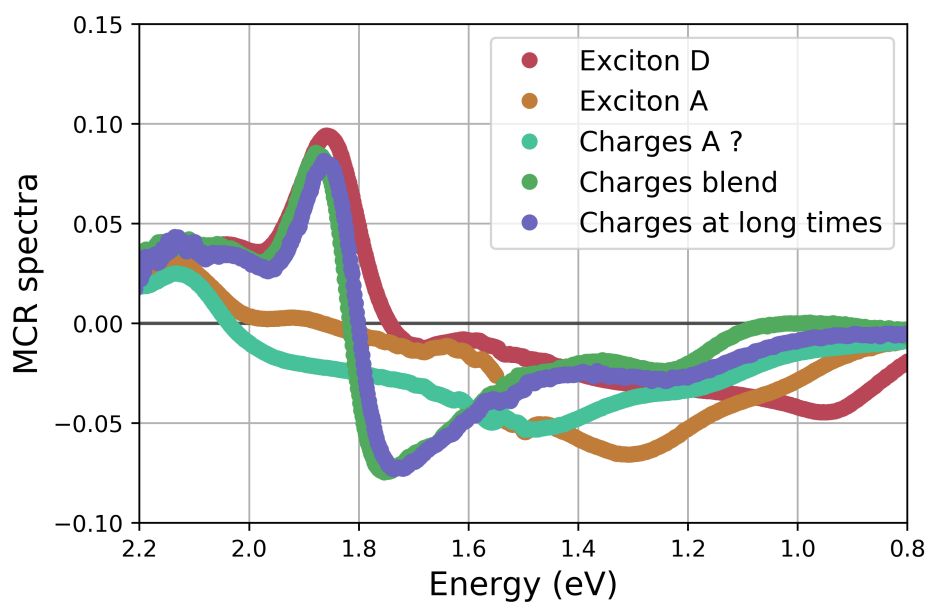

Figure 5.10: MCR spectral components in a film of PPDT2FBT:NIDCS-HO, obatined by soft-modelling, including the TAS spectra at longer time delays.

\section{PPDT2FBT:N2200}

TAS measurements in the all-polymer PPDT2FBT:N2200 blend were carried out under the same conditions as for the previous blends. TA spectral traces at different time delays are shown in Figure 5.11, for time delays up to $6 \mathrm{~ns}$ in (A) and up to $3 \mu \mathrm{s}$ in (B). The spectra measured in a neat donor film is superimposed to show the overlap with the TA spectra of the blend at early times. Therefore, the first component is attributed to singlet excitons in PPDT2FBT. At $532 \mathrm{~nm}$ the absorption from ground state N2200 is neglegible and therefore excitons are created in the donor PPDT2FBT exclusively. 

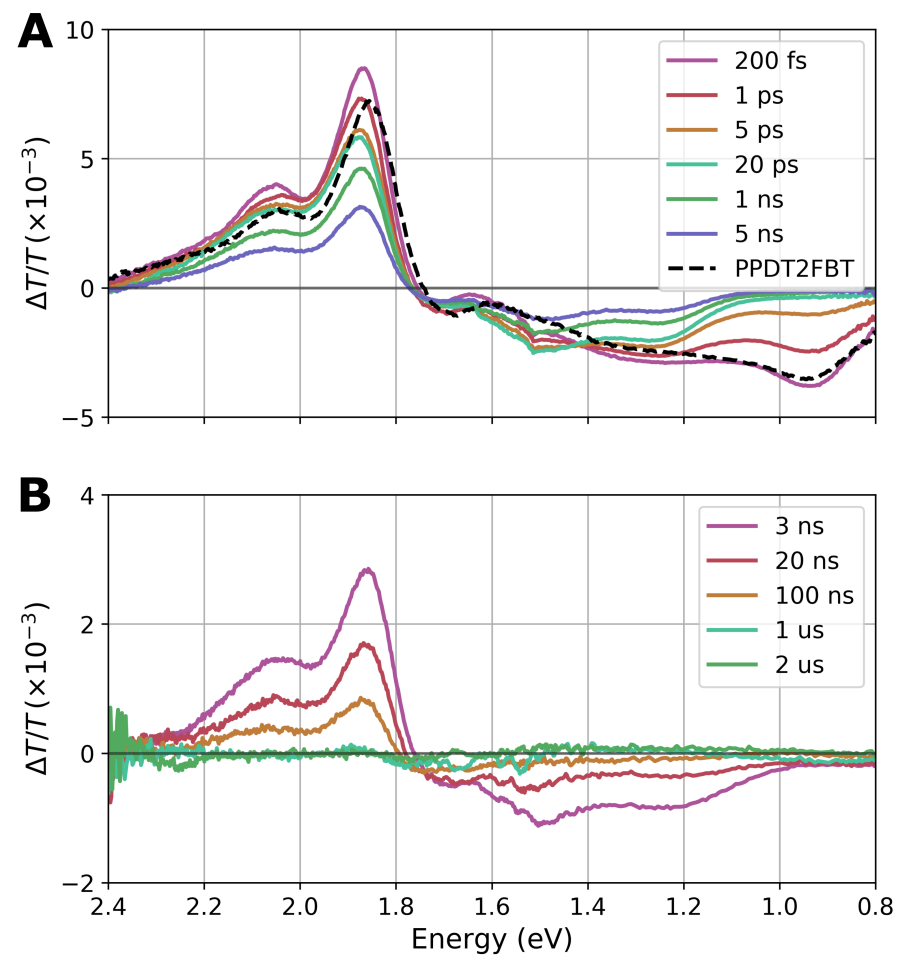

Figure 5.11: TA spectral slices of a PPDT2FBT:N2200 film at indicated time delays.

SVD suggests the presence of a second component, and MCR-ALS was used to disentangle the two species. MCR spectra are shown in Figure 5.12 and spectra at the microsecond timescale is included to show that it matches the second component at early time delays. Therefore, such component was labelled as a charge signal. 


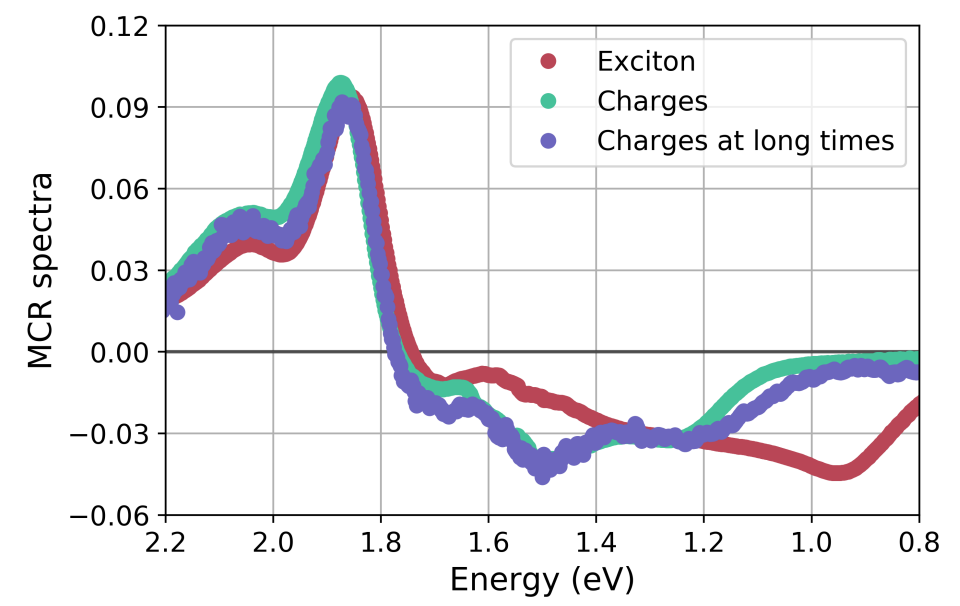

Figure 5.12: MCR spectral components in a film of PPDT2FBT:N2200, obatined by soft-modelling, including the TAS spectra at longer time delays.

\section{Charge spectra comparison}

In Figure 5.13 we compare the charge spectra extracted via the MCR-ALS deconvolution in all three blends using (A) $532 \mathrm{~nm}$ and (B) $670 \mathrm{~nm}$ pump pulses, and we point out the best possible assignment of the PIA feactures. In Figure $5.13 \mathrm{~A}$, the PIA peak at $1.2 \mathrm{eV}$ has the same magnitude with respect to the GSB for all three samples and it is attributed to the hole polaron $\mathrm{D}^{+}$. As discussed earlier, when using $532 \mathrm{~nm}$ pump pulses, spectral masks were used for excitons in PPDT2FBT and NIDCS-HO and also for a charge component in neat NIDCSHO. Therefore, it is not surprising that the remaining charge component in the PPDT2FBT:NIDCS-HO blend corresponds to the donor hole polaron $\mathrm{D}^{+}$only. In PPDT2FBT:N2200, we did not use a spectral mask for charges in the acceptor, which explains the contribution of the $\mathrm{N}^{2} 200^{-}$anion to the MCR charge spectra around $1.5 \mathrm{eV}$, in accordance with the literature. In the fullerene blend the hole polaron is dominant, as previously discussed. When using $670 \mathrm{~nm}$ pump pulses (Figure $5.13 \mathrm{~B}$ ) we additionally observe a peak around $1.5 \mathrm{eV}$ from the 
NIDCS-HO ${ }^{-}$polaron, because the NIDCS-HO charge spectra was not used as a spectral mask in this case. A sharp negative feature is observed to the red side of the GSB, which is stronger for the blends featuring the $\mathrm{PC}_{70} \mathrm{BM}$ and NIDCS-HO acceptors. This signal is known as electro-absorption (EA) and it is caused by the electric fields of photo-generated electron-hole pairs at the donor:acceptor interface affecting the absorption of surrounding chromophores (Stark effect) [145]. The intensity of the EA signal is determined mainly by the electron-hole distance, the alignment of the chromophores with respect to the interfacial dipoles from charge pairs and the nature of the chromophores [139], which explains why the strengh of the EA feature is different for each of our blends. In the PPDT2FBT:N2200 sample, we note that the GSB for N2200 is, according to the literature, within the range $1.51-2.06 \mathrm{eV}$, which explains the lower magnitude in the region of the EA signal.

In the following sections we focus on the dynamics. In Section 5.3.6 the MCR kinetics at short times is explored to investigate the charge generation process and in Section 5.3.7 the long time data collected using $532 \mathrm{~nm}$ pump pulses is analysed to investigate charge recombination.
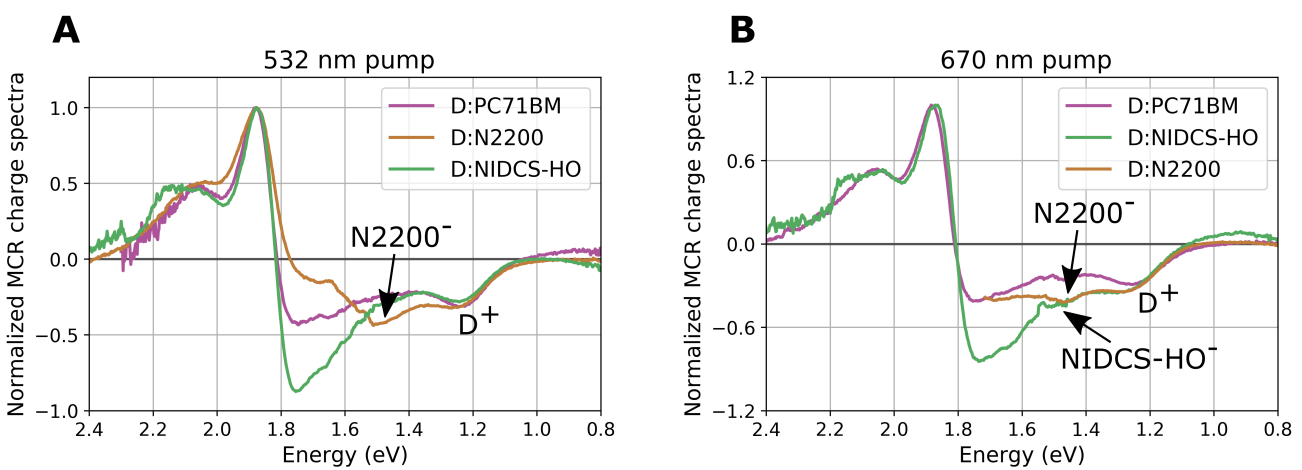

Figure 5.13: Comparison of charge spectra in all three blend films extracted from TA experiments using A) $532 \mathrm{~nm}$ and B) $670 \mathrm{~nm}$ pump pulses. 


\subsubsection{Donor:acceptor blend films - Charge generation}

The temporal resolution of our TA experiments is sufficient to probe the ultrafast process of charge generation. In the previous section we showed that the deconvolution of TA measurements via the MCR bilinear decomposition allows us to obtain the pure spectra of the transient components. In this section we show the MCR kinetics associated with those MCR spectra previously shown.

Figure 5.14 shows the MCR kinetics of the different transient components that emerge upon excitation with 532 and $670 \mathrm{~nm}$ pump pulses in PPDT2FBT:PC 70 BM, PPDT2FBT:NIDCS-HO and PPDT2FBT:N2200 films at a low fluence of $2.6 \mu \mathrm{J} / \mathrm{cm}^{2}$. Additionally, insets show the integrated GSB kinetics for each blend. For PPDT2FBT:PC ${ }_{70} \mathrm{BM}$ the decay of excitons, leads to the growth of a new component, assigned as charges in the donor (including both CT states and free separated charges). The inset in Figure 5.14 A shows a raise of the GSB signal between 10 ps and $1 \mathrm{~ns}$, whereas in Figure 5.14 B the GSB continuously decays. Due to the significant absorption of PCBM at $532 \mathrm{~nm}$ we attribute the GSB raise to hole transfer from acceptor to donor, which is delayed by the diffusion of PCBM excitons to the donor:acceptor interface [148] [146] [147]. This also explains why the charge component in the donor appears to decay earlier when using $670 \mathrm{~nm}$ pulses. At such excitation wavelength, only the donor is excited. Excitons reach the interfase and undergo electron transfer, creating CT states which subsequently either dissociate into free separated charges or recombine. The kinetics of charges in the donor shown in Figure 5.14 B indicates that recombination occurs at times above $100 \mathrm{ps}$. At $532 \mathrm{~nm}$ excitation wavelength, however, excitons are created in both donor and acceptor phases. With hole transfer being delayed due to the diffusion of excitons in the acceptor phase, as hypothesised earlier, charges in the donor phase are created upon hole transfer 
and therefore the overall charge kinetics in the donor continues to grow (up to $1 \mathrm{~ns})$. In Figure $5.14 \mathrm{C}$ for the PPDT2FBT:NIDCS-HO blend excited at $532 \mathrm{~nm}$, the exciton components in donor and acceptor are quenched while other two components emerge. One component is the charge feature which was also measured in the acceptor blend, and the remainder component is assigned to charges in the donor material. An important question here is if charge species in the acceptor are contributing to the photocurrent, which will be discussed in the following paragraph by analysing how the peak value of the charge component in the donor varies with the number of absorbed photons. As in the fullerene blend, the GSB Figure 5.14 C grows due to delayed hole transfer. In the case of the all polymer PPDT2FBT:N2200 blend (Figures 5.14 E and F), we observe that the charge feature begins to decay at earlier times around $30 \mathrm{ps}$, suggesting stronger geminate recombination, and that both the charge feature and the GSB decay continuously regardless of the excitation wavelength. 

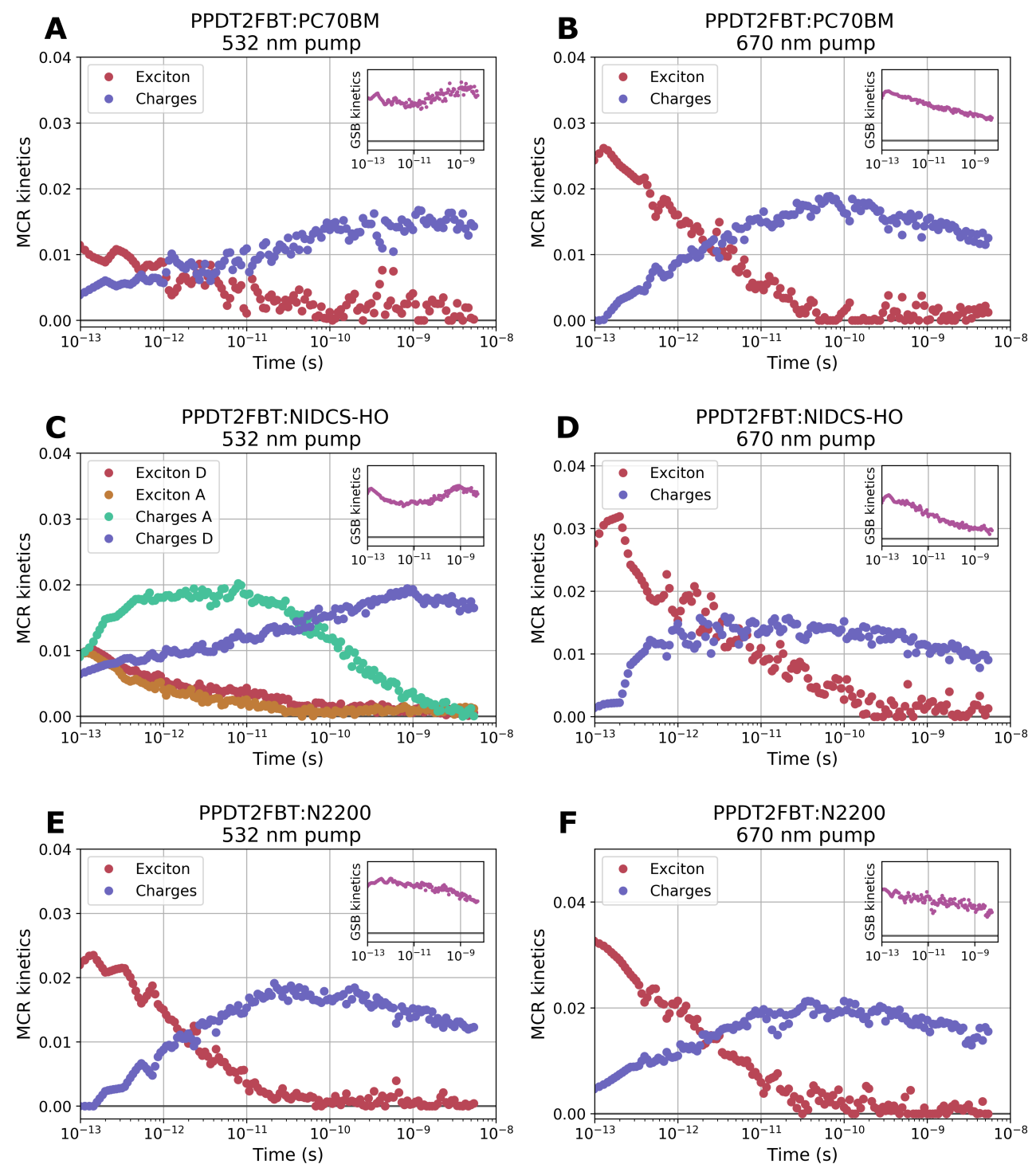

Figure 5.14: MCR kinetics and insets showing the integrated GSB kinetics for A-B) PPDT2FBT:PC ${ }_{70}$ BM, C-D) PPDT2FBT:NIDCS-HO and E-F) PPDT2FBT:N2200 using 532 and $670 \mathrm{~nm}$ pump pulses.

Having isolated the contribution of hole polarons in the kinetics, the effi- 
ciency for the conversion of excitons to charges can be estimated by plotting the peak value of the charge signal against the number of photons absorbed by the film [61]. Assuming that the exciton-to-charge efficiency is unitary in the case of the fullerene blend, the slope in the case of this film is an estimation for the polaron absorption cross-section. For the other two films, the slope will be an estimation of the absorption cross-section multiplied by the exciton-to-charge efficiency. Figure 5.15 shows that in the low intensity region, where the relation charge peak-absorbed photons is linear, the slope in the nonfullerene films does not differ much from the case of the fullerene blend film. However, the slope is slightly lower in the case of PPDT2FBT:NIDCS-HO, suggesting a lower exciton-to-charge yield. In this way, we propose that delayed hole transfer and the formation of charges in relatively large NIDCS-HO domains in the blend results in a slightly lower contribution to the photocurrent, reflected by a lower exciton-to-charge ratio. Cross-sections were obtained from linear regression, returning a value of $\sigma=1.6 \times 10^{-16} \mathrm{~cm}^{2}$ for the fullerene blend. In the case of the PPDT2FBT:NIDCS-HO blend, where more photons are absorbed, the relation charge peak-absorbed photons is no longer linear at the highest pump fluence used in our experiments, due to some degree of recombination in such conditions. 


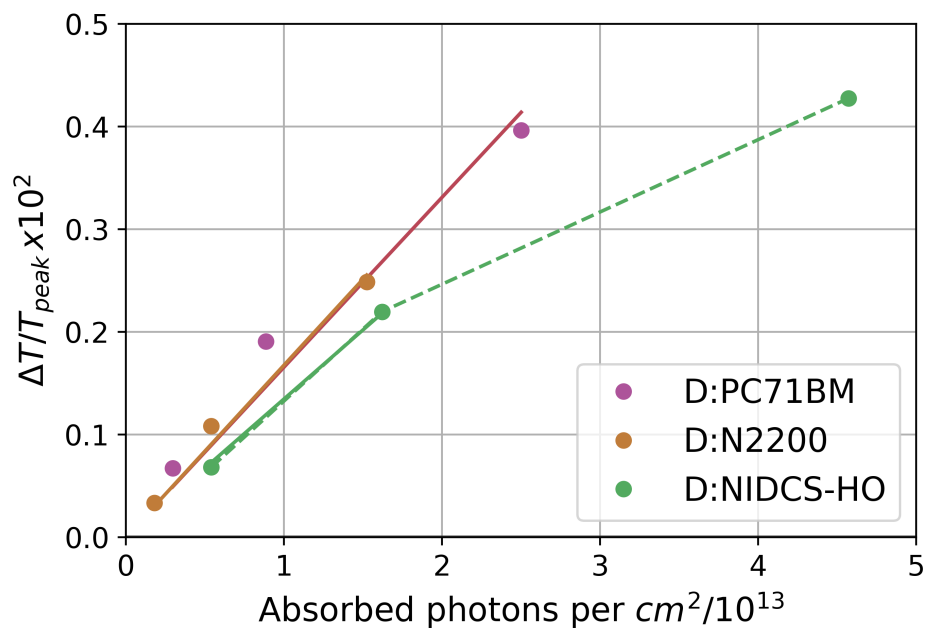

Figure 5.15: Peak value of the charge signal against the number of photons absorbed per $\mathrm{cm}^{2}$ for each film.

\subsubsection{Donor:acceptor blend films - Charge recombination}

Our TAS experiments were measured on samples without any electrodes, therefore at $V_{o c}$ condition, where all charges eventually recombine.

Above the nanosecond timescale, charge dynamics can be obtained by simple direct integration over the PIA region of the $D^{+}$feature, without the need of a bilinear decomposition. Considering that the absorption bands of transient CT states and hole polarons typically overlap, such species cannot be distinguished as unique TAS features [29]. However, because CT states recombine monomolecularly whereas separated charges follow recombination kinetical orders equal or higher than 2, they may be isolated via fluence-dependent measurements using an appropriate kinetic model. This approach will be explored in the following paragraphs.

The method implemented here to disentangle the temporal evolution of bound and separated polarons, named here as CT and SC, consists of using a 
model to describe the rate of change of these species, and optimise their profiles $\mathrm{CT}(\mathrm{t})$ and SC(t) such that the square of the residuals are minimised across measurements at different pump densities. The model is composed of vibrationally cold CT states which dissociate into SCs and SCs recombining nongeminately to the CT state, with all recombination to the ground state occurring via cold CT states at the donor-acceptor interfaces. This model reminds the Onsager-Braun model and is described by the system of Equations 6.1 and a scheme is shown in Figure 5.16.

The Onsager-Braun model was developed by Braun to describe the charge generation process in donor:acceptor organic semiconductors, based on the Onsager theory for the dissociation and recombination of ions in solution [65]. In this scenario, illustrated in Figure 5.16, separated charges generated from the dissociation of CT states undergo transport (drift and diffusion) to either reach the collecting electrodes (desired process) or cancel out (recombine) when encountering an opposite charge (undesired process). The yield of free carriers was specified by Braun as the product of the dissociation rate $k_{d}$ and the lifetime of the CT states, i.e. $\frac{k_{d}}{k_{d}+k_{r}}$ [65]. The main limitation of this model to describe the dissociation and recombination processes occuring in bulk-heterojunction OPVs is that the model assumes that carriers exist in a single phase, whereas holes and electrons in a bulk-heterojunction OPV live in different phases (holes in the donor phase and electrons in the acceptor phase).

The nongeminate recombination term in Equations 6.1 is described with a recombination constant $\gamma$ and recombination order $\lambda+1$, which is equal to two when the recombination process is bimolecular. Such recombination process is also analog to the recombination of ions in solution, which can be described by the Langevin theory, where the recombination rate depends on the sum 
of electron and hole mobilities and the recombination process is bimolecular. However, recombination orders in bulk-heterojunction OPV have been found to be higher than two. Different explanations for higher-order recombination include the trapping of charges in the tail states of the DOS and mobilities being charge carrier density dependent.

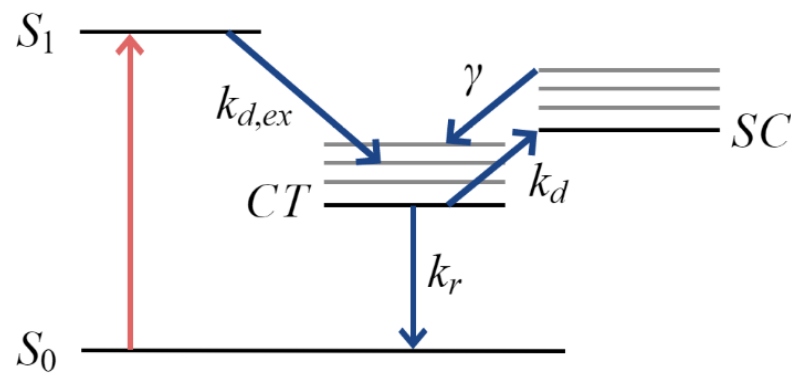

Figure 5.16: Scheme of the kinetic model described by the system of Equations 6.1.

$$
\left\{\begin{array}{l}
\frac{d C T(t)}{d t}=-k_{d} C T(t)-k_{r} C T(t)+\gamma S C(t)^{\lambda+1} \\
\frac{d S C(t)}{d t}=k_{d} C T(t)-\gamma S C(t)^{\lambda+1}
\end{array}\right.
$$

Since no analytical solution is found for $\mathrm{CT}(\mathrm{t})$ and $\mathrm{SC}(\mathrm{t})$ satisfying Equations 6.1 [61], kinetic profiles are solved numerically assuming that all kinetic parameters are time-independent. The best kinetic parameters are obtained by the simultaneous fit of the charge decay at different fluences. Prior to global fitting, TA charge signals were converted into charge densities using the cross-section value estimated for each blend in Section 5.3.6, through the following expression 5.2 for $\Delta T / T$ deduced from the Lambert-Beer law within the small signal approximation [149].

$$
\Delta T / T=-\sigma N d
$$


Table 5.2: Space of possible values for each parameter in the model described by the system of Equations 6.1 and illustrated in Figure 5.16.

\begin{tabular}{lc}
\hline & Parameter space \\
\hline$k_{r}\left(\mathrm{~s}^{-1}\right)$ & $1: 15 \times 10^{8}$ \\
\hline$k_{d}\left(\mathrm{~s}^{-1}\right)$ & $1: 10 \times 10^{9}$ \\
\hline$\lambda+1$ & $2: 3$ \\
\hline
\end{tabular}

where $\sigma$ is the cross-section of the electronic transition (in $\mathrm{cm}^{2}$ ), $N$ is the charge density (in $\mathrm{cm}^{-3}$ ) and $d$ is the film thickness (in $\mathrm{cm}$ ).

The space of possible values for each parameter in the model, presented in Table 5.2, was specified taking into consideration kinetic parameters found in the literature, such as the values of $k_{r}$ and $\lambda+1$ for RR-P3HT:PCBM, PCPDTBT:PCBM and TQ1:N2200 shown in Table 2.2 of this thesis.

In principle, the best kinetic parameters can be obtained by ordinary leastsquares, minimising the sum of the squares of the residuals between the data and the model, with guess initial values and bounds on the variables. However, we found that the output parameters are sensitive to the choice of the initial values, indicating the presence of several local minima. To avoid this problem, global optimization was achieved by applying the differential evolution algorithm by Storn and Price [150] from the Python library scipy.optimize. Our code allows us to fix parameters in the model to static values, for example, we can set the recombination order to a value of two, to evaluate the fitting outputs considering nongeminate recombination as a bimolecular process.

Figure 5.17 shows the resulting fitting curves with solid lines and the corre- 
sponding output parameters are given in Table 5.3. Dashed-line curves in Figure 5.17 correspond to the global fit where the recombination order is fixed to a value of two, i.e. assuming that recombination is bimolecular. 

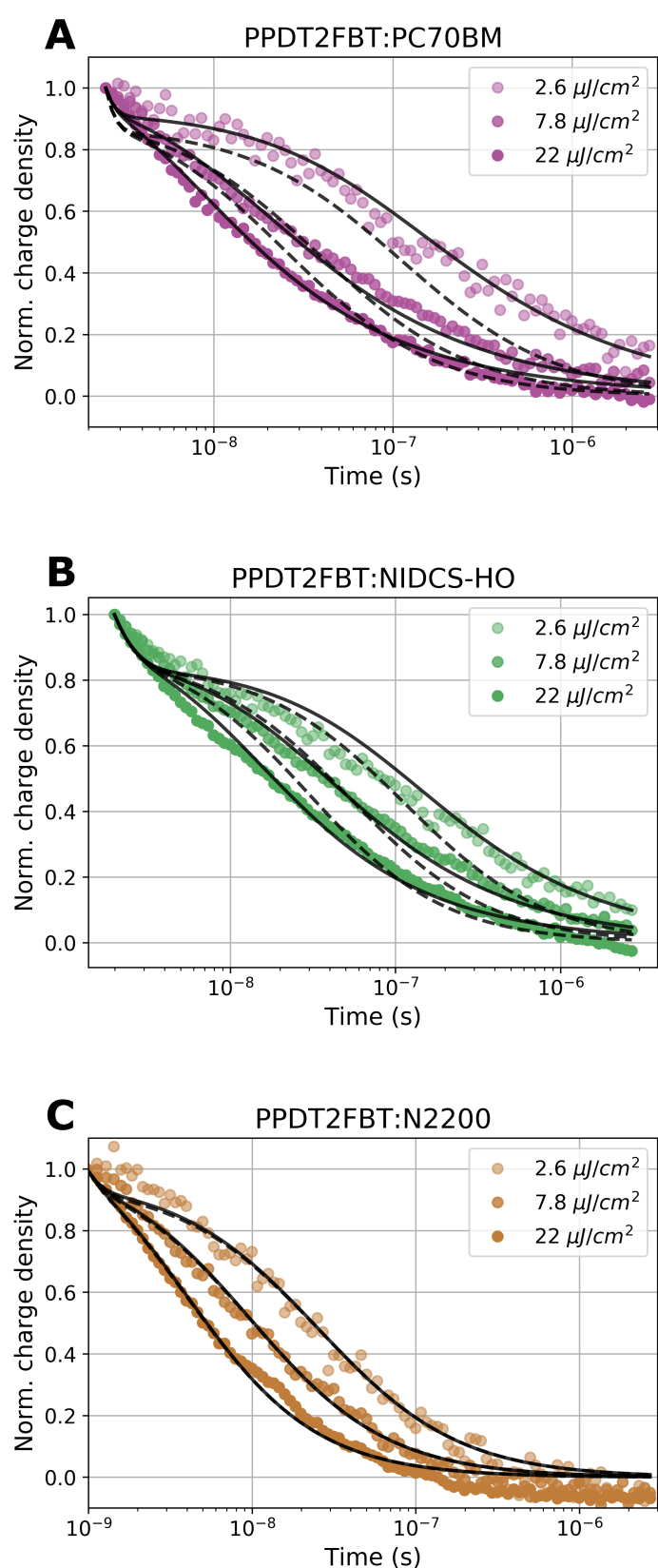

Figure 5.17: Charge decays at different fluences and global fitting curves for PPDT2FBT:PCBM (top panel), PPDT2FBT:NIDCS-HO (middle panel) and PPDT2FBT:N2200 (low panel).

In the fullerene blend (Figure $5.17 \mathrm{~A}$ ), decay of the CT state to the ground 
Table 5.3: Output kinetic parameters from the global fit of the fluence dependent charge decays.

PPDT2FBT : PCBM PPDT2FBT : NIDCS-HO

PPDT2FBT : N2200

\begin{tabular}{cccc}
\hline$k_{r}\left(\mathrm{~s}^{-1}\right)$ & $5.8 \times 10^{8}$ & $3.3 \times 10^{8}$ & $1.4 \times 10^{9}$ \\
\hline$k_{d}\left(\mathrm{~s}^{-1}\right)$ & $2.7 \times 10^{9}$ & $1.2 \times 10^{9}$ & $2.9 \times 10^{9}$ \\
\hline$\frac{k_{d}}{k_{r}+k_{d}}$ & 0.83 & 0.78 & 0.67 \\
\hline$\beta\left(\mathrm{cm}^{3} \mathrm{~s}^{-1}\right)$ & $1.0 \times 10^{-11}$ & $5.2 \times 10^{-12}$ & $8.2 \times 10^{-10}$ \\
\hline$\lambda+1$ & 2.8 & 2.6 & 2.0 \\
\hline
\end{tabular}

state exhibits a lifetime of $1 / k_{r}=1.7 \mathrm{~ns}$ and the estimated CT dissociation yield is 0.83 , which is above and in good agreement with the EQE $=0.8$ of this blend at $532 \mathrm{~nm}$ (Table 6.1). The recombination order results to be larger than 2, i.e. nongeminate recombination is not bimolecular. This might suggest that due to disorder, carriers are trapped in the density of states and the recombination of free charges with trapped charges is an additional recombination pathway to bimolecular recombination between free charges. In order to compare our experimental nongeminate constant with the Langevin value, an effective constant $\beta$, equivalent to a recombination order of 2 , is calculated as $\beta=\gamma \times N^{\lambda-1}$ with carrier density $N$ at solar illumination $\left(N=5 \times 10^{15} \mathrm{~cm}^{-3}\right) . \beta=1.0 \times 10^{-11} \mathrm{~cm}^{3} \mathrm{~s}^{-1}$ results to be lower than the calculated Langevin constant, which for this film is $2.7 \times 10^{-9} \mathrm{~cm}^{3} \mathrm{~s}^{-1}$ considering the sum of the hole and electron mobilities, and $9.0 \times 10^{-10} \mathrm{~cm}^{3} \mathrm{~s}^{-1}$ considering the slowest charge carrier only, i.e. using the expression in Equation 2.16 [151]. Overall, these results are in good agreement with published results in other fullerene systems [61].

The nonfullerene PPDT2FBT:NIDCS-HO blend shows a CT lifetime of $1 / k_{r}=$ 
$3 \mathrm{~ns}$, which is longer than in the fullerene blend. We relate this to the energy levels of this acceptor, leading to a higher energy of the CT state. We have recently demonstrated, for low donor content blends, that a higher lying CT state results in a reduced nonradiative CT decay rate [63]. Thus, it is not surprising to measure a slower geminate recombination rate in PPDT2FBT:NIDCS-HO. The estimated dissociation yield of 0.78 is in good agreement with the EQE $=0.7$ of this blend at $532 \mathrm{~nm}$ (Table 6.1). Regarding nongeminate recombination, the recombination order is also higher than 2 , which might suggest the contribution of trapped assisted recombination due to disorder. The effective bimolecular constant $\beta=5.2 \times 10^{-12} \mathrm{~cm}^{3} \mathrm{~s}^{-1}$ is lower than the calculated Langevin constant, which for this film is $8.5 \times 10^{-11} \mathrm{~cm}^{3} \mathrm{~s}^{-1}$ considering the sum of the hole and electron mobilities, and $3.8 \times 10^{-11} \mathrm{~cm}^{3} \mathrm{~s}^{-1}$ considering the slowest charge carrier only [151]. Interestingly, this value of the effective recombination constant is on the order of magnitude of the recommended value for achieving best performance NFA devices that has been recently published [136]. Due to the potential for tunability in NFA acceptors, we can use our kinetic model to vary some of the kinetic parameters in a reasonable way, and analyse the outputs to propose ways in which the performance could be improved. For example, we can run our numerical model fixing the effective bimolecular constant to the value we have obtained, modifying the recombination order to a bimolecular process. In this way, we keep the benefitial low value of $\beta$ we have obtained for this system, and we verify how much the lifetime of charges is increased if there was no disorder. In Figure 5.18 we show that if recombination is only bimolecular and the parameters $k_{r}, k_{d}$ and $\beta$ are maintained, charges would live approximately an order of magnitude longer, increasing their chance of being extracted in an operating device. 


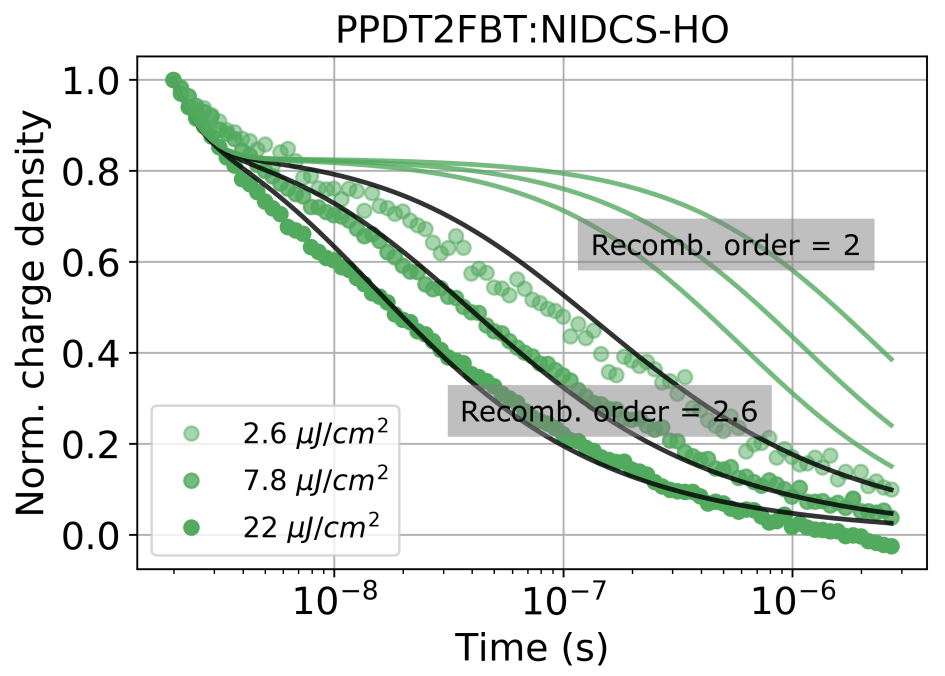

Figure 5.18: Global fitting curves for PPDT2FBT:NIDCS-HO where the effective bimolecular constant was fixed to the value reported in Table 5.3 and the recombination order is changed to a value of 2 .

The polymer:polymer blend PPDT2FBT:N200 Table 5.3 shows that the decay of the CT state to the ground state exhibits a lifetime of $1 / k_{r}=0.7 \mathrm{~ns}$, which is lower than the lifetime in the other blends, suggesting strong geminate recombination. The estimated dissociation yield of 0.67 is above the $\mathrm{EQE}=0.4$ of this blend at $532 \mathrm{~nm}$ (Table 6.1). The recombination order results to be close to 2 (the value for 3D Langevin recombination) and the effective bimolecular constant $\beta=8.2 \times 10^{-10} \mathrm{~cm}^{3} \mathrm{~s}^{-1}$ has the same order of magnitude as the calculated Langevin constant, which for this film is $1.3 \times 10^{-10} \mathrm{~cm}^{3} \mathrm{~s}^{-1}$ considering the sum of the hole and electron mobilities, and higher than $9.0 \times 10^{-12} \mathrm{~cm}^{3} \mathrm{~s}^{-1}$ which is the value considering the slowest charge carrier only [151]. The effective bimolecular recombination $\beta$ is on the order of magnitude of the one measured by TDCF in TQ1:N2200 systems, however it is one order of magnitude higher than the value of $\beta$ obtained by TAS in the same systems using a two pool kinetic 
model for the kinetic rate of CT and SCs [62].

\subsection{Conclusions}

In this work we compared charge generation and recombination between three systems featuring the same polymer donor PPDT2FBT matched with three different acceptors, namely the fullerene acceptor $\mathrm{PC}_{70} \mathrm{BM}$, the small molecule nonfullerene acceptor NIDCS-HO and the polymeric acceptor N2200. Our results provide insight on the processes that limit the performance of each device.

Our comparative TAS results show that in the fullerene and NIDCS-HO based devices hole transfer from acceptor to donor give rise to a delayed increase of charges in the donor phase. However, the advantage of this contribution to charges that could potentially be extracted in a functional device, is limited by the morphology in the NIDCS-HO -based device, as evidenced by a lower exciton-to-charge yield. This is in agreement with a relative low value of $J_{s c}$ in this system, despite its complementary donor-acceptor steady state absorption.

Our analysis of the charge recombination process consisted on global fitting the charge decay at different pump fluences using a kinetic model that involves the recombination of separated charges via the CT state. Constraining our parameter space to values that are found in the literature, our model fits the data very well when the recombination order is a free parameter larger or equal than 2. The dissociation yields calculated as $\frac{k_{d}}{k_{r}+k_{d}}$ follow the same trend as the EQE for these systems at the energy of our pump pulses. Importantly, the lifetime of the CT state to the ground state transition, i.e. $\frac{1}{k_{r}}$, is considerably longer for the NIDCS-HO blend compared to the other blends, which is consistent with the 
higher energy of the CT state due to the lower energy offset of this system. The lower value for $\frac{1}{k_{r}}$ is found in the PPDT2FBT:N2200 blend. We therefore identify that geminate recombination is a strong limiting factor in the all-polymer blend.

An effective bimolecular recombination constant $\beta$ was calculated for each blend and it is found to be lowest for the NIDCS-HO blend and highest for the N2200 all-polymer blend. Maintaining the effective bimolecular constant that we have obtained for PPDT2FBT:NIDCS-HO, we evaluated how the kinetics changes if there was no disorder and the recombination order was actually equal to two. Our result in Figure 5.18 shows that in absence of disorder, charges would live longer before recombining, increasing their chance of being extracted in an operating device. Thus, we speculate that disorder is the main limiting factor in this system. 


\section{Chapter 6}

\section{Charge Photogeneration in}

\section{Low-Donor-Content Organic}

\section{Photovoltaic Blends}

\subsection{Overview}

Establishing a fundamental picture for the generation of free charges in organic solar cells require an understanding of the dynamical properties of tightly bound, interfacial charge-transfer (CT) states at the donor-acceptor heterojunction. In this work we study charge carrier dynamics in a series of four solar devices composed predominantly of $\mathrm{C}_{60}(94 \mathrm{~mol} \%)$ and small amounts of organic small molecule donors (6mol\%) where their CT state energies are systematically varied. The well-defined microstructure of these systems, makes it easier to correlate macroscopic device properties to molecular parameters.

We use broadband transient absorption spectroscopy (TAS) to study charge recombination kinetics and we find that a kinetic model to describe the compe- 
tition between CT dissociation and recombination, including CT reformation through the bimolecular recombination of free charge, is a well suited framework for these systems. The kinetic parameters we retrieve via a global fit show that the systems with low CT energy exhibit short-lived CT states, likely related to their high nonradiative losses. These results are consistent with a combination of time-delayed collection field (TDCF), and external quantum efficiency (EQE) measurements at different bias performed by our collaborators, which show that the voltage dependence of the photocurrent is more pronounced for the systems with low CT energies and that this voltage dependence follows closely the bias dependence of charge generation, identifying geminate recombination as a major loss. Furthermore, we find that the dynamics of the CT decay are connected to the CT state energy via the energy-gap law. The main implication of our results is that the energy of the CT state is identified as the main parameter determining the efficiency of photocurrent generation in these morphologically well-defined donor-acceptor blends [63].

This work has been published in the Journal of the American Chemical Society (JACS) under the title Energy-Gap Law for Photocurrent Generation in FullereneBased Organic Solar Cells: The Case of Low-Donor-Content Blends [63].

\subsection{Background}

\subsubsection{Low-donor-content organic photovoltaic blends}

Conclusions regarding the role of a given parameter on the efficiency of photocurrent generation are often deduced from the study of donor-acceptor bulk heterojunction systems, where the energetics is varied systematically through chemical modifications. However, the morphology of such systems is generally 
ill-defined and, more importantly, may undergo changes when modifying the chemical structure of the components forming the blend. This ambiguity of interpretation is avoided in solar cells comprising mainly the fullerene acceptor, with small molecule donors added at low concentration $(<10 \mathrm{~mol} \%)$. Surprisingly, their efficiencies can be relatively high, despite the limited absorption of the fullerene [152-155]. Such low-donor-content systems have a well-defined morphology, where the donor molecules are homogeneously dispersed in the fullerene matrix [156-158]. This presents a very clear advantage for fundamental studies, as the variation of the chemical structure of the small molecule donor does not result in relevant changes of the blend microstructures. Building upon this advantage, low-donor-content blends have been investigated to correlate macroscopic device properties to molecular parameters, and to understand such correlations on the basis of the underlying dynamic processes [68, 159-162].

\subsubsection{Nonradiative voltage losses}

Voltage losses are considered as the limiting factor in the performance of fullerene-based organic photovoltaic devices, including losses due to i) the energy offsets at the heterojunction, and ii) fast charge recombination [52]. Although certain systems have shown high performace with very small donoracceptor energy offsets (driving force) [59], losses due to radiative and nonradiative recombination remain strong. Radiative voltage losses are inevitable given that such transitions are directly related to the inverse process of absorption, and indeed the Shockley-Queisser limit for the maximum efficiency assumes the contribution from radiative recombination exclusively. However, real devices additionally exhibit significant nonradiative voltage losses.

A recent relevant finding by Vandewal at al., based on 100 donor-fullerene 
combinations comprising vacuum- and solution-processed devices in different architectures, was that nonradiative recombination increased with decreasing CT energy of the donor-fullerene blends, as shown in Figure 6.2 [68]. The authors argue that this correlation is associated with an increased wavefunction overlap between the relaxed CT state and higher order vibrational modes of the ground state, in analogy with the energy-gap law for nonradiative decay in large organic molecules and their complexes. They carried out replacement of carbon-hydrogen bonds by carbon-deuterium bonds, which led to excluding $\mathrm{C}-\mathrm{H}$ vibrations as the dominant vibrational mode and they explain that analysis of the low-energy tail of CT emission spectra points to skeletal carbon-carbon bonds as the main mediator for the nonradiative decay of $\mathrm{D}-\mathrm{C}_{60} \mathrm{CT}$ states. This type of nonradiative recombination would therefore be intrinsic to organic materials. The authors report that if nonradiative voltage losses are taken into account, the optimal optical gap is blueshifted by $0.2-0.3 \mathrm{eV}$ and the theoretical maximum PCE is reduced to about $25 \%$ for single-junction OPVs, compared with the values calculated in Shockley-Queisser conditions. 

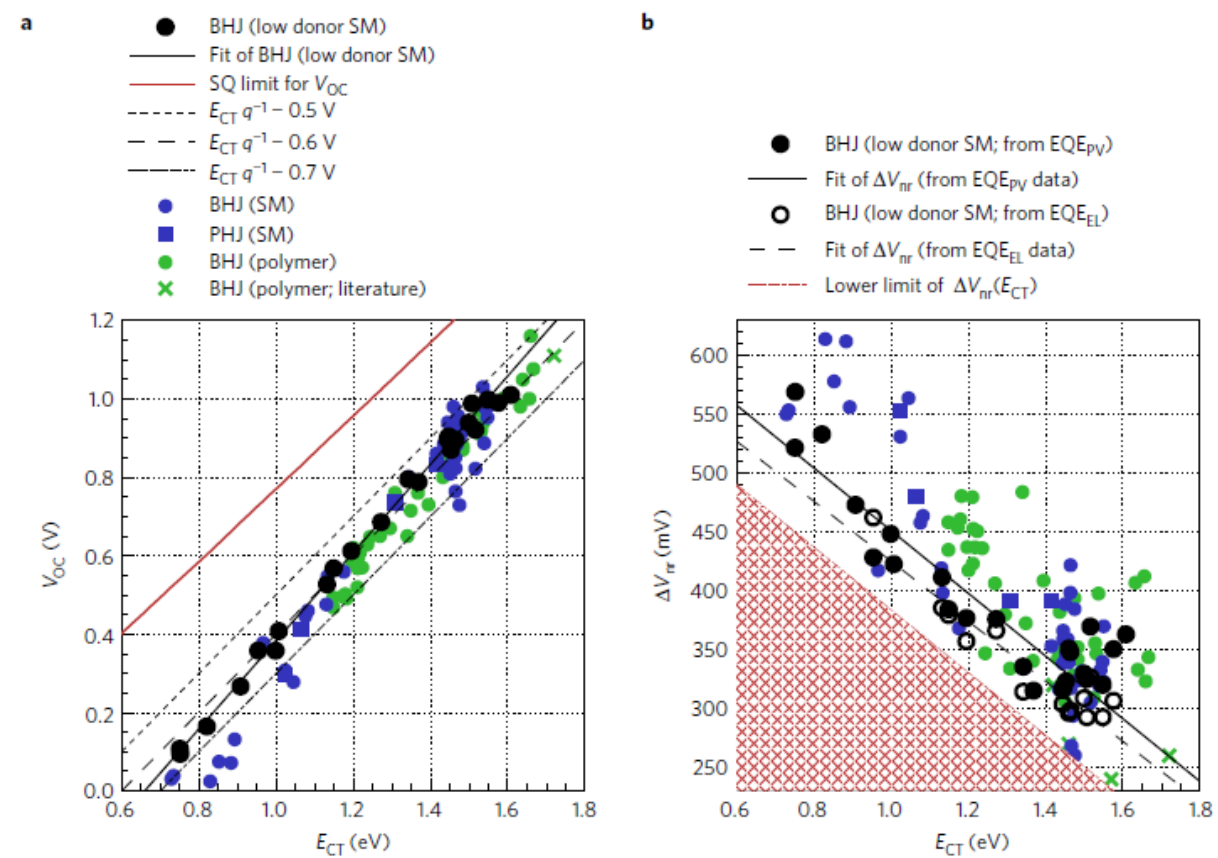

Figure 6.1: a) $V_{O C}$ and b) nonradiative voltage losses as a function of $E_{C T}$ in a wide range of OPV devices. Figure reproduced with permission from Reference [68].

\subsection{Results and discussion}

In this work, we study charge carrier dynamics in selected devices composed predominantly of $\mathrm{C}_{60}(94 \mathrm{~mol} \%)$ and small amounts of organic small molecule donors (6mol\%), with their CT state energies systematically varied. Since our TAS measurements on these systems were performed within a collaborative work with the Neher group at Postdam University, this section begins with a summary of the photovoltaic characteristics of the devices and the results obtained by our collaborators. 


\subsubsection{Device performance versus open-circuit voltage}

Our devices consist of a $50 \mathrm{~nm}$ of active layer, sandwiched between $\mathrm{ITO} / \mathrm{MoO}_{3}$ and Bphen/Ag electrodes. Figure 6.2 shows the J-V curves of several devices and the chemical structures of four selected donors studied in greater detail here and Table 6.1 summarizes their photovoltaic parameters. Despite the devices absorbing the same number of photons, it is striking that all device parameters, i.e., $J_{S C}, V_{O C}$, and fill factor $(F F)$, are very sensitive to the choice of donor utilized.
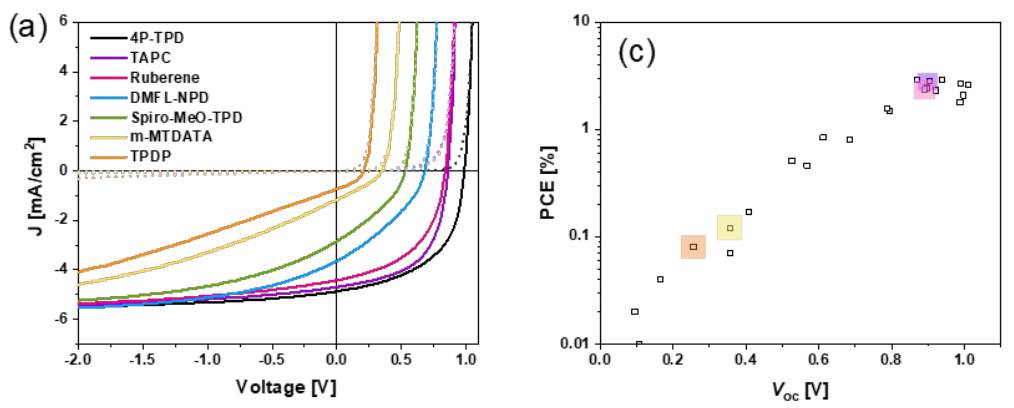

(b)
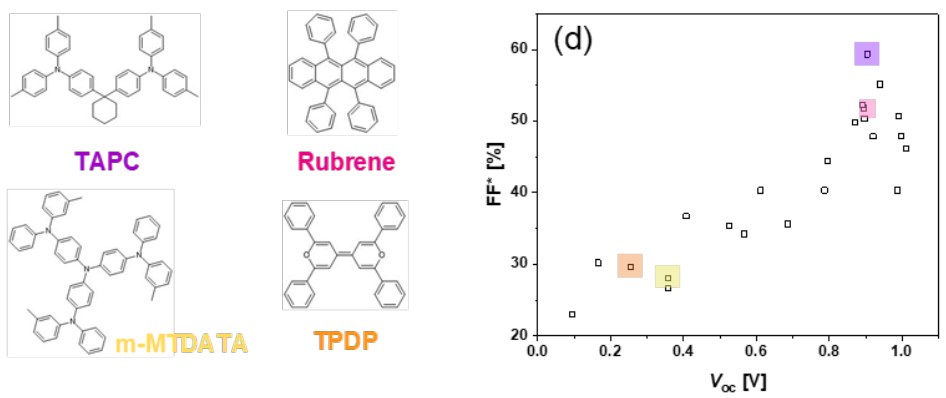

Figure 6.2: (a) J-V characteristics under simulated AM1.5G illumination (solid curves) and in the dark (dashed lines) for different low-donor content blends. (b) Chemical structure of some selected donor molecules. (c) Power conversion efficiency (PCE) as a function of the open-circuit voltage $\left(V_{O C}\right)$ for a wide range of donor molecules. Highlighted in color is the performance of the blends with the donors shown in (b). (d) Effective fill factor $\left(\mathrm{FF}^{*}\right)$ plotted versus $V_{O C}$. Figure from our collaborators, reprinted with permission from [63]. Copyright 2019 American Chemical Society. 
Table 6.1: Photovoltaic parameters, CT state properties, and $\mu_{h}$ for selected Donor: $\mathrm{C}_{60}$ systems [63].

\begin{tabular}{ccccc}
\hline & TAPC & Rubrene & m-MTDATA & TPDP \\
\hline$V_{o c}(\mathrm{~V})$ & 0.9 & 0.9 & 0.36 & 0.26 \\
\hline$J_{s c}\left(\mathrm{mAcm}^{-2}\right)$ & 5.3 & 5.22 & 1.05 & 0.81 \\
\hline $\mathrm{FF}(\%)$ & 58.5 & 50.7 & 32.3 & 39.6 \\
\hline $\mathrm{FF}^{*}(\%)$ & 59.3 & 51.8 & 28.0 & 29.6 \\
\hline $\mathrm{PCE}(\%)$ & 2.8 & 2.37 & 0.12 & 0.08 \\
\hline $\mathrm{E}_{C T}(\mathrm{eV})$ & 1.45 & 1.46 & 0.95 & 0.91 \\
\hline$\lambda(\mathrm{eV})$ & 0.16 & 0.08 & 0.41 & 0.16 \\
\hline$\mu_{h}\left(\mathrm{~cm}^{2} \mathrm{~V}^{-1} \mathrm{~s}^{-1}\right)$ & $1.57 \times 10^{-5}$ & $6.61 \times 10^{-5}$ & $1.08 \times 10^{-8}$ & $2.42 \times 10^{-5}$ \\
\hline
\end{tabular}

Importantly, a decrease in $V_{O C}$ goes along with a drastic reduction in $\mathrm{FF}$ as can be deduced from the J-V curves in Figure 6.2. In general, the FF is determined by the voltage dependence of geminate and nongeminate recombination processes, but it also depends explicitly on the value of $V_{O C}$. Therefore, an effective fill factor, $\mathrm{FF}^{*}$, was calculated by shifting all J-V curves so that they all have a $V_{O C}$ of $1 \mathrm{~V}$. The dependence of $\mathrm{FF}^{*}$ on the original (real) $V_{O C}$ is shown in Figure $6.2 \mathrm{~d}$, highlighting the continuous deterioration of the J-V characteristics with decreasing $V_{O C}$.

In light of this evidence, we carried out a detailed investigation of the photocurrent generation mechanisms for four selected blends, TAPC: $\mathrm{C}_{60}$, rubrene: $\mathrm{C}_{60}, \mathrm{~m}-\mathrm{MTDATA}: \mathrm{C}_{60}$ and TPDP: $\mathrm{C}_{60}$, guided by their specific values of $V_{O C}, \mathrm{FF}^{*}$, and $\mu_{h}$. TAPC and rubrene both have high $E_{C T}$ (and $V_{O C}$ ) values, 
while m-MTDATA and TPDP stand out because of their small $E_{C T}$ and their very different $\mu_{h}$.

Subsequently, the EQE spectra was measured at different bias in the four selected blends, finding that their bias dependence is different to that of a $\mathrm{C}_{60}$ device and specific to each blend. The results show that irrespective of whether the initially photoexcited species is an intermolecular CT state in neat $\mathrm{C}_{60}$, an intramolecular $\mathrm{C}_{60}$ Frenkel exciton, or a low energy donor-acceptor CT state, the EQE displays the very same dependence on the internal electric field. A similar situation has been reported for various donor-acceptor blends in the past and assigned to charge generation via low-energy donor-acceptor CT states.

\subsubsection{Bias and photon energy dependence of free charge gener- ation}

Bias dependence of charge generation and of the steady state photocurrent were compared by our collaborators, by overlaying the external generation efficiency (EGE) calculated from time-delayed collection field (TDCF) measurements onto the J-V curves at reverse bias, as shown in Figure 6.3 for each blend. For the TAPC and rubrene devices (high $V_{O C}$ systems), EGEs are nearly independent of bias except at around $V_{O C}$ where the internal electric field drops near to zero and nongeminate recombination becomes important. For TPDP, however, the shape of the J-V curve is fully governed by the bias-dependence of the EGE, implying that geminate recombination limits the photocurrent in this system. The situation for m-MTDATA appears to be an intermediate case, where biasdependent charge generation is accompanied by nongeminate recombination losses resulting from the very low hole mobility of this particular blend. 


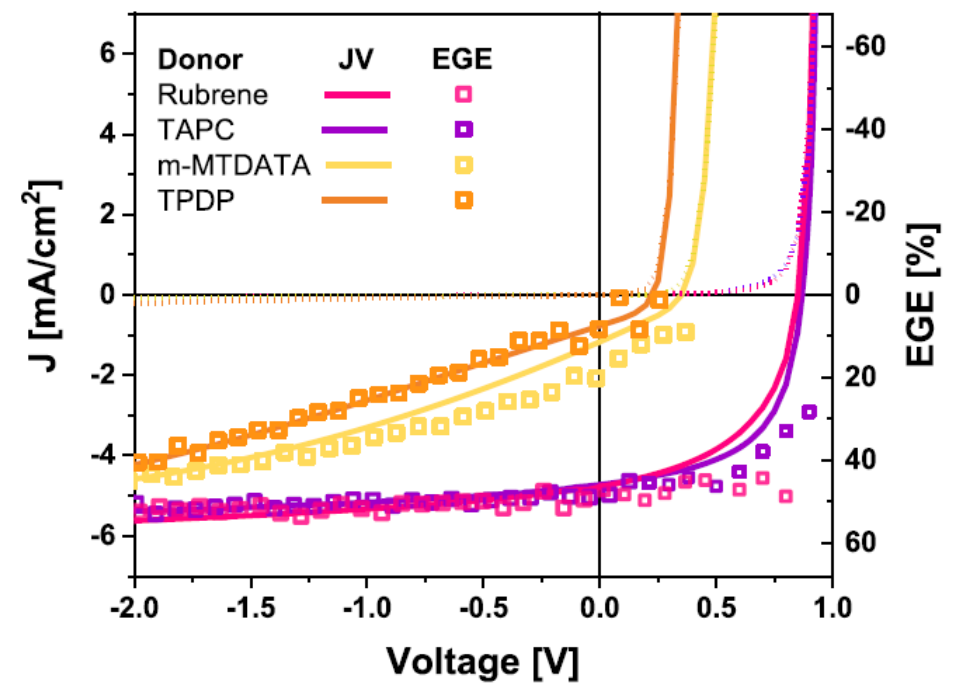

Figure 6.3: External generation efficiency (EGE) from TDCF measurements, plotted as a function of bias voltage for four selected low-donor-content blends, and compared to the corresponding J-V curves. Figure from our collaborators, reprinted with permission from [63]. Copyright 2019 American Chemical Society.

Additionally, our collaborators carried out TDCF measurements using different excitation wavelengths, to test whether the nature of the primary excited species has an appreciable effect on the free charge generation efficiency. Results show that for all systems, there is no systematic effect of the excitation energy on the bias dependence of the generation efficiency, confirming the conclusion from the bias-dependent EQE experiments that photocurrent generation involves a low energy donor-acceptor CT state manifold.

From the experimental EGE data, our collaborators deduced the CT dissociation efficiency as a function of the internal field and Monte Carlo simulations were performed to fit the experimental dissociation probabilities, by varying only the CT decay rate $k_{r}$. Excellent fits were obtained despite the fact that all input parameters except $k_{r}$ were the same for all blends. This confirms our 
hypothesis that the pathway of CT dissociation is largely independent of the choice of the donor.

\subsubsection{Transient absorption spectroscopy}

To directly measure the dynamics of photoexcited species, in particular the fate of CT states and free charges, we applied transient absorption spectroscopy (TAS) to the four selected low-donor-content blends: TAPC: $\mathrm{C}_{60}$, rubrene: $\mathrm{C}_{60}$, m-MTDATA: $\mathrm{C}_{60}$ and TPDP: $\mathrm{C}_{60}$. Experiments were performed with an excitation wavelength of $400 \mathrm{~nm}(3.1 \mathrm{eV})$, which excites mainly $\mathrm{C}_{60}$. Such pump pulses were tuned using a parametric amplifier (TOPAS), and the broadband probe was generated by focusing one of the $800 \mathrm{~nm}$ arms of the setup onto a $3 \mathrm{~mm}$ YAG crystal. More details about our transient absorption spectrometer are described in chapter 3. As mentioned earlier, the samples consisted on encapsulated active layers of $50 \mathrm{~nm}$ thickness composed of vacuum-deposited $\mathrm{C}_{60}$ and small amounts of the organic donor molecules.

\subsubsection{Spectral assignments}

We present in Figure 6.4 the TAS data obtained for pristine $\mathrm{C}_{60}$ at different time delays with an excitation wavelength of $400 \mathrm{~nm}$. When the data matrix is factorised via SVD, the magnitude of the singular values suggest that two components are needed to explain the data, which is in accordance with the literature. The first component is associated with intermolecular CT excitons formed in $\mathrm{C}_{60}$, which explains the reasonable device performances obtained in the neat material, whereas the second component is caused by localised Frenkel excitons. In Reference [163] Banerji et al. measured the TAS dynamics of such species in thermally evaporated $\mathrm{C}_{60}$ films using different excitation wavelengths 
to selectively excite the different species. When excited with $450 \mathrm{~nm}$ pump pulses, both Frenkel and intermolecular CT excitons are generated in $\mathrm{C}_{60}$, in accordance with our measurements. At early time delays the TAS signal exhibits a broad photoinduced absorption (PIA) band together with a pronounced EA feature, caused by strong electric dipoles perturbing the CT transitions of the surrounding $\mathrm{C}_{60}$ aggregates. Such spectrum is assigned to the direct population of intermolecular CT states. Subsequently, the TAS spectra shows a strong decay of the EA feature within the first picoseconds, after which mainly Frenkel excitons are left. Excitons are characterized by the weak $\mathrm{C}_{60}$ GSB below $500 \mathrm{~nm}$ strongly masked by overlapping PIA bands, a broad PIA band from 500-1200 nm, an EA peak around $550 \mathrm{~nm}$ and a weak indent at $650-850 \mathrm{~nm}$ due to SE. In our study, we can disregard the first transient component, i.e. the intermolecular CT state, since we will focus on the data above the picosecond time scale, where recombination processes in the blends take place.

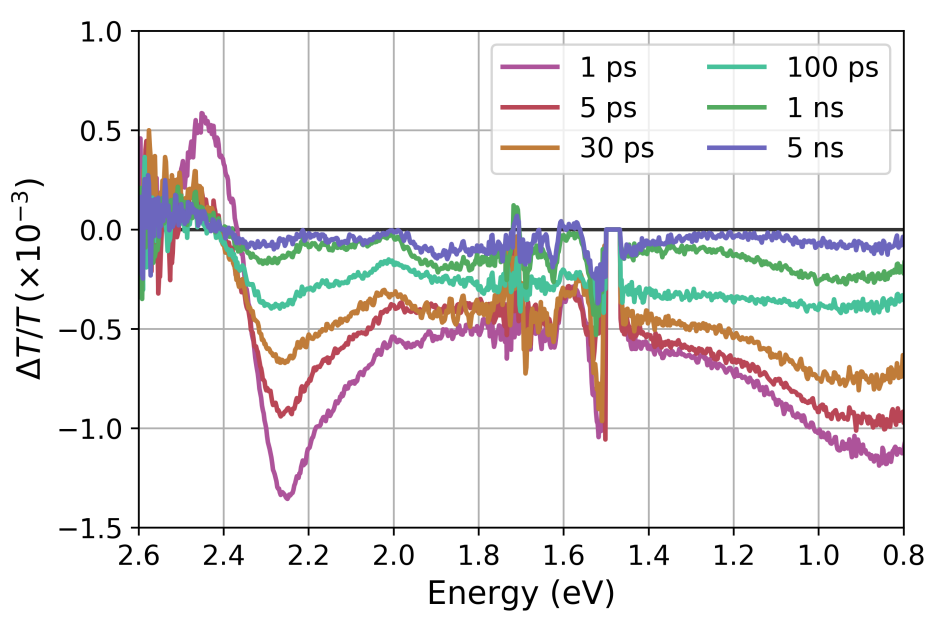

Figure 6.4: $\mathrm{TA}$ spectral slices of a neat $\mathrm{C}_{60}$ film at indicated time delays.

Figure 6.5 shows spectral slices of the TAS data of pristine $\mathrm{C}_{60}$ in the spectral region that is dominated by photoinduced absorption, and considering time 
delays above the first picosecond, where only Frenkel excitons remain present. Since the EA feature depends strongly on the ordering of the molecules at the interface, it results appropriate to avoid this spectral region when comparing the spectra of neat $\mathrm{C}_{60}$ spectra with the spectra in the blends. Singular value decomposition of the data shown in Figure 6.5 confirms that the data matrix can be fully explained by a single component.

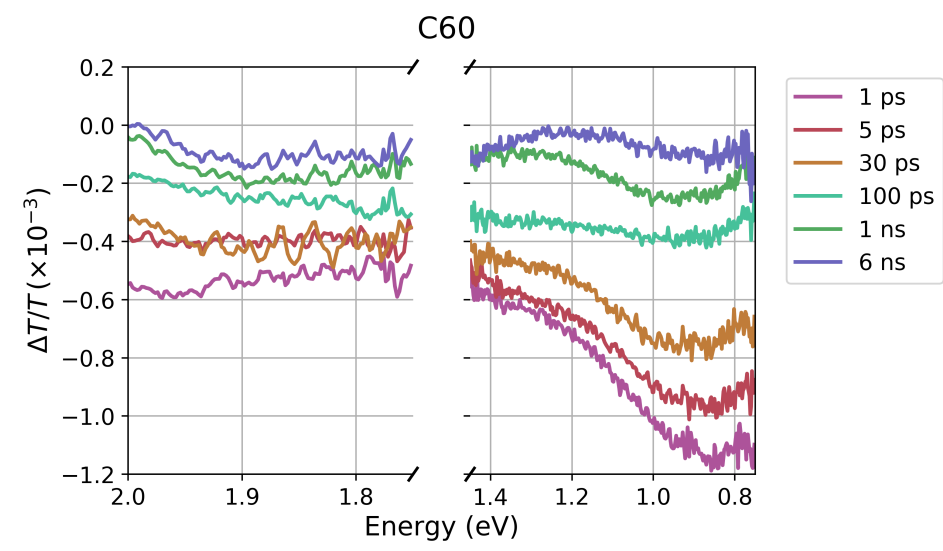

Figure 6.5: $\mathrm{TA}$ spectral slices of the neat $\mathrm{C}_{60}$ film at indicated time delays, in the spectral region that is dominated by photoinduced absorption, and considering time delays above the first picosecond, where only Frenkel excitons remain present.

Figure 6.6 shows spectral slices of the TAS data obtained for A) TAPC: $\mathrm{C}_{60}$, B) rubrene: $\left.\mathrm{C}_{60}, \mathrm{C}\right) \mathrm{m}-\mathrm{MTDATA}: \mathrm{C}_{60}$, and D) TPDP: $\mathrm{C}_{60}$ at indicated time delays, along with the exciton spectra measured in neat $\mathrm{C}_{60}$. We observe a clear contribution from the $\mathrm{C}_{60}$ exciton to the early time spectra in the TAPC: $\mathrm{C}_{60}$ and rubrene: $\mathrm{C}_{60}$ blends. In the case of $\mathrm{m}-\mathrm{MTDATA}: \mathrm{C}_{60}$ and TPDP: $\mathrm{C}_{60}$, the overlap with the $\mathrm{C}_{60}$ spectrum is weaker, due to another component having spectral features in the same region as the $\mathrm{C}_{60}$ exciton, as will be seen below. We considered the $\mathrm{C}_{60}$ spectrum as a known spectrum in our analysis on the blends (i.e. as a spectral mask) and we confirmed that the remainder spectrum we retrieved 
matched the nanosecond timescale spectrum, where excitons are no longer present.
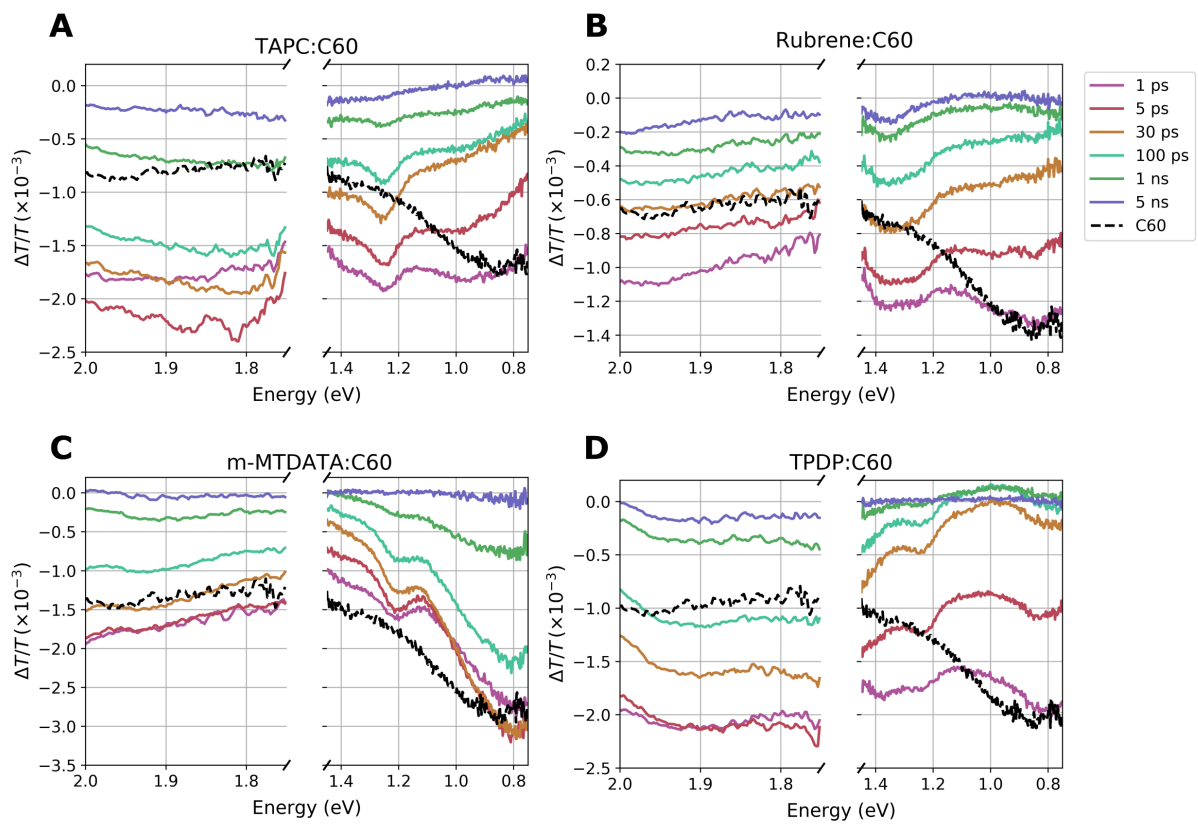

Figure 6.6: TA spectral slices of the blends A) TAPC: $\left.\mathrm{C}_{60}, B\right)$ rubrene: $\left.\mathrm{C}_{60}, \mathrm{C}\right) \mathrm{m}$ MTDATA: $\mathrm{C}_{60}$, and D) TPDP: $\mathrm{C}_{60}$ at indicated time delays.

In previous chapters we have shown that dynamics of the transient species composing a TA measurement can be retrieved via one of the following approaches i) finding a spectral region where only one component is present and simply integrate the signal over that region, or ii) deconvolute different overlaping signals via a bilinear decomposition. Due to the absence of clean regions with a single component contribution, we have followed the second approach by applying the soft modeling technique MCR-ALS (multivariate curve resolution by alternating least-squares) to decompose the data into a bilinear model, i.e., pairs of spectra and kinetics for each transient species. The TAS data for pristine $\mathrm{C}_{60}$ shown earlier was used as a spectral mask for the blends, allowing the 
spectra and kinetics of charges (including both CT states and SCs) to be cleanly isolated without rotational ambiguity. Additionally we used constraints for nonnegativity of the concentration profiles and non-positivity of PIA signals. This approach was implemented for all blends, except for m-MTDATA. The behavior of m-MTDATA is different, because the charge spectrum includes a feature in the same region as the exciton feature, which prevents the deconvolution of these two profiles to be viable through the MCR-ALS method. Thus, our approach for m-MTDATA consisted of obtaining the charge kinetics by direct integration of the decaying signal, bearing in mind that the spectrum of charges cannot be disentangled from the exciton spectrum. Interestingly, at very high fluences our measurements show intensity dependence on the exciton PIA spectra, and as a result we found that the exciton mask had to be used at a matching fluence. However, at the fluences presented here, the exciton spectra at the different fluences are not noticeably different.

The deconvolution of the transient components, and direct integration of the decaying signal in the case of m-MTDATA, returns the charge components in each blend. The spectral assignment of free charges was supported by confirming the $\mathrm{C}_{60}$ anion feature at around $1.2 \mathrm{eV}$ present in each case [164] and by comparing the spectra to the absorption spectra of $\mathrm{FeCl}_{3}$-doped donor solutions [63]. The charge features that we identify are shown in Figure 6.7. 


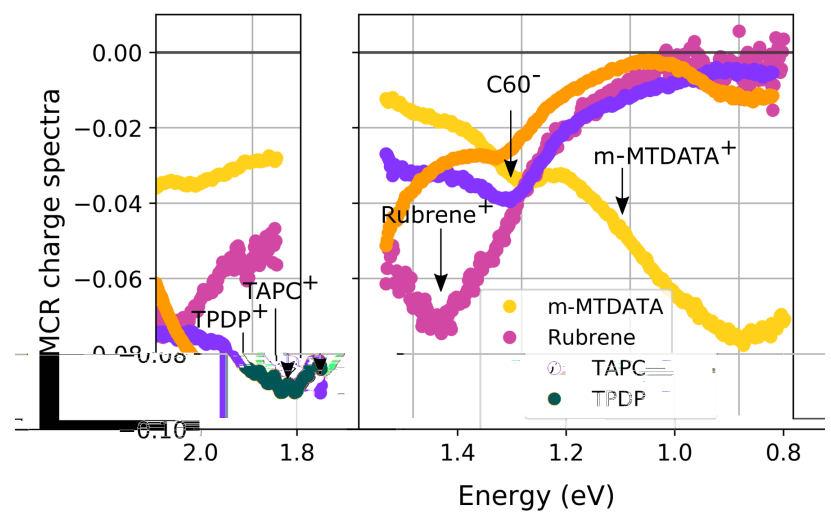

Figure 6.7: Spectra of the charge component in each blend, extracted via MCR-ALS, along with the positions of absorption peaks in FeCl3-doped donor solutions.

\subsubsection{Charge carrier dynamics}

Charge spectra shown for each blend in Figure 6.7 is accompanied by a corresponding kinetic profile. As noted earlier in this thesis, TA spectra of charges does not distinguish CT and SC species, however, the behavior of the CT states may be isolated via fluence-dependent measurements. The normalized charge kinetics shown in Figure 6.9 surprisingly reveal a minor fluence dependence on the nanosecond time scale, indicating that a first-order recombination process dominates the fate of the photogenerated carriers at this fluences. We argue below that this kinetic scenario stems from the rapid reformation of CT states from free charges through efficient bimolecular recombination, which enables the CT lifetime to be recovered from the observed recombination kinetics.

To model the dynamics of charge decay, we used the simple rate model in Equations 6.1 to describe the competition between CT dissociation and recombination, including CT reformation through the bimolecular recombination of free charge with the encounter coefficient $k_{\text {enc }}$. 


$$
\left\{\begin{array}{l}
\frac{d C T(t)}{d t}=-k_{d} C T(t)-k_{r} C T(t)+\gamma S C(t)^{2} \\
\frac{d S C(t)}{d t}=k_{d} C T(t)-\gamma S C(t)^{2}
\end{array}\right.
$$

In this model, nongeminate recombination of SC is bimolecular, all recombination to the ground state is via the decay of CT states with the CT recombination rate $k_{r}$, and $k_{d}$ is the zero field CT dissociation rate. We are aware that this rate model oversimplifies the complex process of CT dissociation and reformation via multiple carrier hopping. Nevertheless, this model allows us to reliably assess trends in CT lifetimes without needing to develop a simulation tool capable of assessing the high carrier densities of our measurements or introducing new parameters that cannot be independently verified. A similar rate model (including also the formation and decay of triplet CT states) was successfully employed to model the TAS transients of different donor/fullerene blends over a wide range in fluence [?]. Since no analytical solution is found for these nonlinear differential equations [61], we performed a numerical global fit of our data in MATLAB using the MATLAB function LSQCURVEFIT which returns the coefficients that best fit the model to all data sets. The initial conditions for SC and CT were determined such that SC tends to zero at early times ( $1 \mathrm{ps}$ ). This was achieved with $81 \%$ of CT and $9 \%$ of SC at a 30 ps time delay.

Because of the nearly complete lack of a fluence dependence of the TAS traces in Figure 6.9, an independent determination of all kinetic parameters in Equation 6.1 is not possible to achieve. Unfortunately, our TAS experiments at lower fluences where BMR dominates the carrier dynamics were very noisy, mainly because of the small thickness of the blend and the low absorption cross section of the fullerene anion. Global fits were, therefore, performed with $k_{d}$ and $k_{\text {enc }}$ being fixed at selected different values. Figure 6.8 shows the results of such 
a fitting where we assumed Langevin type encounter-limited recombination (Equation 2.14) with an electron mobility $\mu_{e}\left(\gg \mu_{h}\right)=0.5 \mathrm{~cm}^{2} /(\mathrm{Vs})$ and a dielectric constant of $\epsilon_{r}=4.5$, which are typical values for C60 fullerene [165, 166], yielding $k_{e n c}=2 \times 10^{-7} \mathrm{~cm}^{3} \mathrm{~s}^{-1}$. The zero field dissociation rate was estimated through $k_{d}=k_{\text {enc }} \frac{3}{4 \pi a^{3}} \exp \left(-E_{b i n d} / k_{B} T\right)$ where $E_{b i n d}=e^{2} / 4 \pi \epsilon_{0} \epsilon_{r} a$ is the CT Coulomb binding energy, and $a$ is the radius of the thermalised CT state, which was fixed to a value of $a=1.5 \mathrm{~nm}$. The fit captures the early temporal decay of the TAS signal, though it deviates from the experimental data at longer delay times. We note that our analysis is rather simple as it does not take into account the molecular details of the blend, energetic disorder, and related dispersive effects. Due to these limitations, the parameters $k_{e n c}$ and $k_{d}$ were modified to obtain a better fitting of the kinetics. For the relatively high value of $k_{\text {enc }}$ considered so far, we found that changing $k_{d}$ does not improve the quality of the fit, and we attribute this to the very rapid bimolecular recombination of SCs to the CT state. 
A

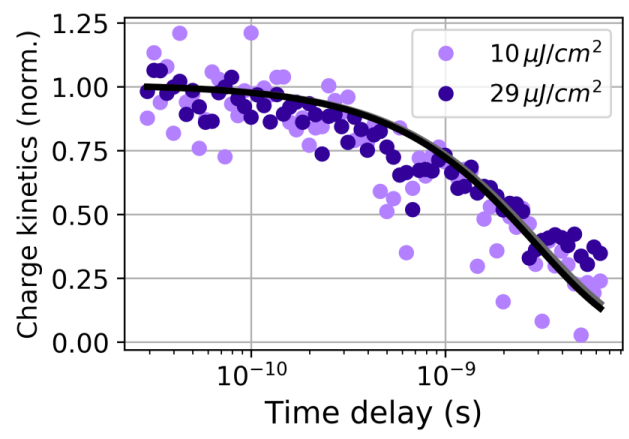

C

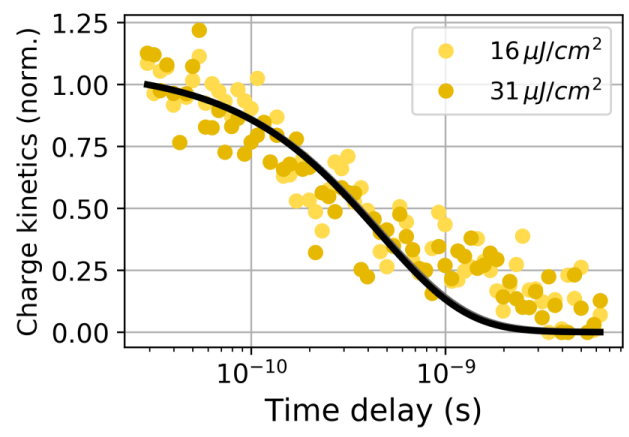

B

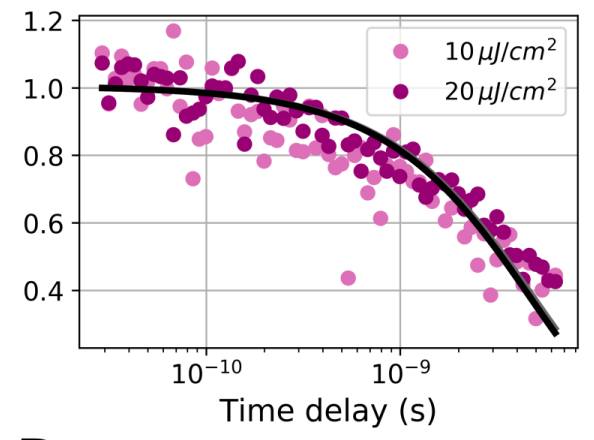

D

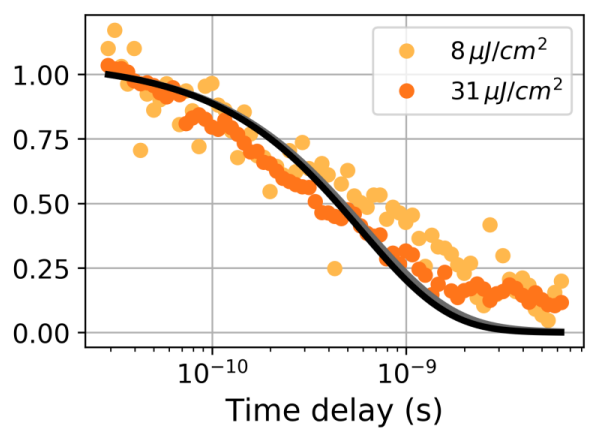

Figure 6.8: Normalized TAS transients of A) TAPC: $\mathrm{C}_{60}$, B) rubrene: $\left.\mathrm{C}_{60}, \mathrm{C}\right) \mathrm{m}$ MTDATA: $\mathrm{C}_{60}$ and D) TPDP: $\mathrm{C}_{60}$, together with the result of the numerical global fitting with Equation 6.1, assuming fast CT dissociation $\left(k_{d}=2.5 \times 10^{9} \mathrm{~s}^{-1}\right)$ and rapid bimolecular recombination of free charges $\left(k_{\text {enc }}=2 \times 10^{-7} \mathrm{~cm}^{3} \mathrm{~s}^{-1}\right)$.

Subsequently, we altered the value of $k_{\text {enc }}$, and Figure 6.9 displays fits where this parameter has been reduced to $k_{\text {enc }}=2 \times 10^{-9} \mathrm{~cm}^{3} \mathrm{~s}^{-1}$, now providing a better quality of the fit. Values for $k_{r}$ from these fits are assembled in Table 6.2. $k_{r}$ increases significantly when going from the rubrene blend to the low CT state energy (and low performance) systems, with the corresponding CT decay times decreasing from few nanoseconds to hundreds of picoseconds. At the same time, values for $k_{r}$ are smaller than the set value of $k_{d}=2.5 \times 10^{9} \mathrm{~s}^{-1}$ for the TAPC and rubrene blends, meaning that dissociation is faster than recombination 
for such blends, whereas the opposite occurs for the m-MTDATA and TPDP blends. This is fully compliant with the interpretation of the EQE and EGE experiments performed by our collaborators in Postdam. However, we note that such comparison should be considered with care, as $k_{d}$ simplifies the complex process of CT dissociation as pointed out above. Figure 6.10 shows how the CT decay times of the different blends vary with different assumptions made for $k_{e n c}$ and that independently of the exact choice of the input parameters the resulting values of $k_{r}$ exhibit a similar 10-fold increase when going from rubrene to TPDP.

A

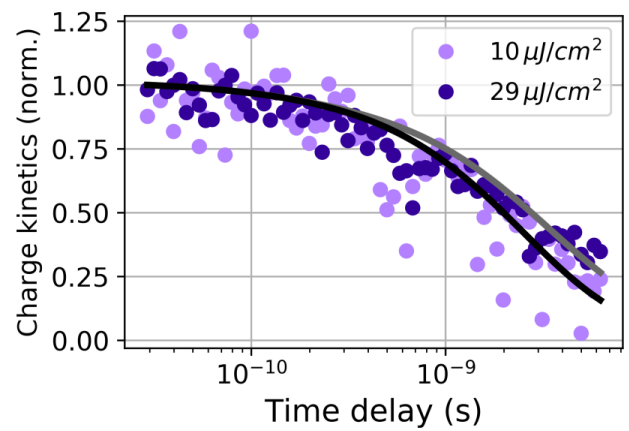

\section{C}

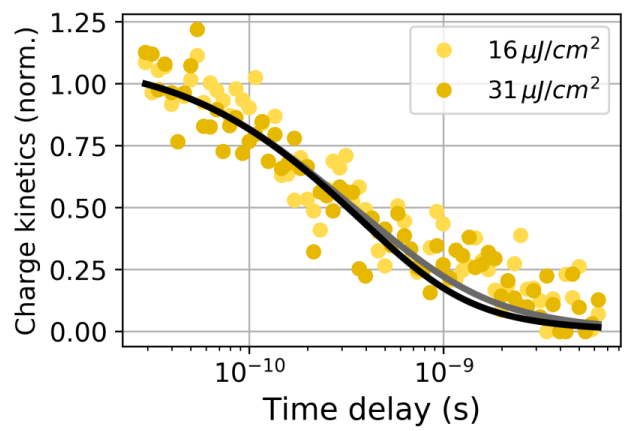

\section{B}

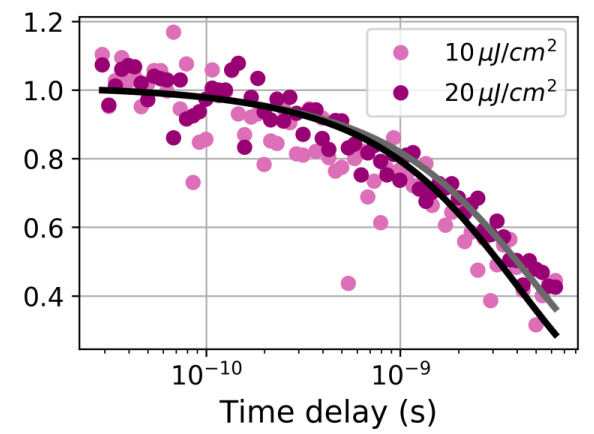

D

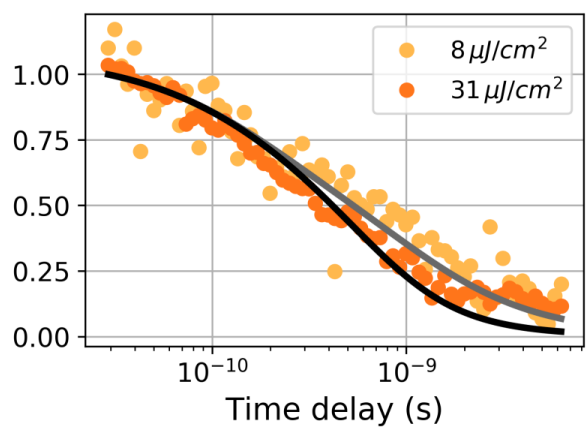

Figure 6.9: Fitting curves from the numerical global fit of Equation 6.1 (solid lines) for A) TAPC: $\left.\mathrm{C}_{60}, \mathrm{~B}\right)$ rubrene: $\left.\mathrm{C}_{60}, \mathrm{C}\right) \mathrm{m}-\mathrm{MTDATA}_{\mathrm{C}} \mathrm{C}_{60}$, and D) TPDP: $\mathrm{C}_{60}$, with all fitting parameters listed in Table 6.2 
Table 6.2: CT Decay rates, $k_{r}$, as estimated from the fit of the fluence dependent TAS measurements in Figure 6.9, with given values for the bimolecular recombination coefficient, $k_{e n c}$, and the zero-field dissociation rate, $k_{d}$ [63].

\begin{tabular}{ccccc}
\hline & TAPC & Rubrene & m-MTDATA & TPDP \\
\hline$k_{\text {enc }}\left(\mathrm{cm}^{-3} \mathrm{~s}^{-1}\right)$ & $2.0 \times 10^{-9}$ & $2.0 \times 10^{-9}$ & $2.0 \times 10^{-9}$ & $2.0 \times 10^{-9}$ \\
\hline$k_{d}(\mathrm{~s}-1)$ & $2.5 \times 10^{9}$ & $2.5 \times 10^{9}$ & $2.5 \times 10^{9}$ & $2.5 \times 10^{9}$ \\
\hline$k_{r}(\mathrm{~s}-1)$ & $0.65 \times 10^{9}$ & $0.44 \times 10^{9}$ & $3.86 \times 10^{9}$ & $2.97 \times 10^{9}$ \\
\hline $1 / k_{r}(\mathrm{~ns})$ & 1.54 & 2.27 & 0.26 & 0.34 \\
\hline
\end{tabular}

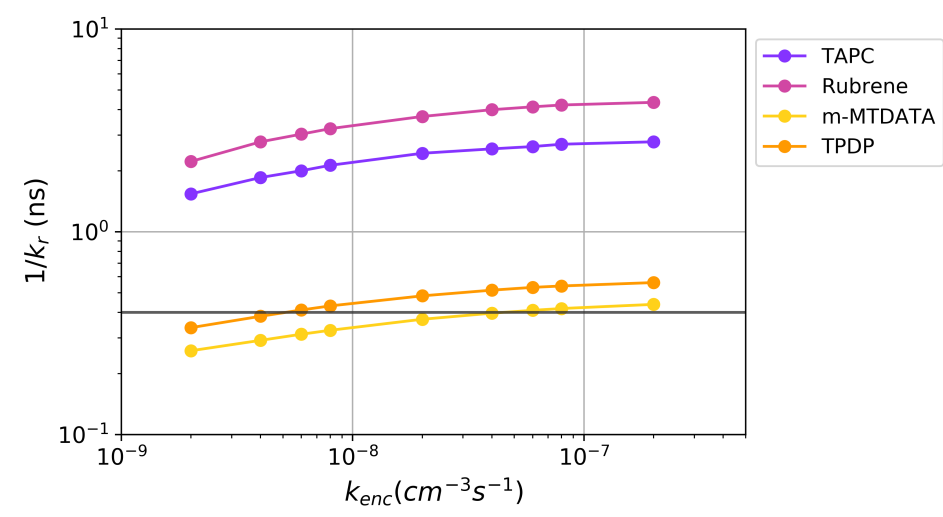

Figure 6.10: CT decay times for the four systems under study and different assumptions made regarding the choice of $k_{e n c}$. The reference line indicates the dissociation lifetime considered $\left(1 / k_{d}=0.4 \mathrm{~ns}\right)$.

So far we have shown that our TAS measurements, in combination with bias dependent EQE and TDCF measurements applied to these samples, provide evidence that the rate of the CT decay depends on the CT energy, and that the poor performance of the devices with low $V_{O C}$ is due to the fast nonradiative CT recombination, competing with CT dissociation. It is a common view that the 
decay of CT states to the ground state in organic donor-acceptor systems occurs primarily through nonradiative decay channels. Recent work proposed that such nonradiative decays are inherent to organic donor-acceptor heterojunctions and that they proceed primarily through internal conversion. In this limit, the CT decay rate is related to $E_{C T}$ and the vibrational energy of the dominant/mean high-frequency mode via the well-known energy-gap law described in Equation 6.2:

$$
k_{r}=k_{\text {rad }}+k_{\text {nonrad }} \cong k_{\text {nonrad }} \propto \exp \left[-B \frac{E_{C T}-\lambda}{h v_{v}}\right]
$$

where $\lambda$ is the CT reorganization energy due to soft (low frequency) modes. The prefactor $B$ in the exponent depends on details of the exact modes involved. Figure 6.11 plots our values for $k_{r}$ (Table 6.2) as a function of $E_{C T}-\lambda$, together with the values of $k_{r}$ from the simulation of the TDCF data. Both data sets align with the prediction of the energy gap law according to Equation 6.2, with $h v_{v} / B \approx 200 \pm 20 \mathrm{meV}$.

We conclude that blends with low-lying CT states (low $V_{O C}$ ) indeed suffer from high CT state decay rates, that the rate of this process is determined by nonradiative internal conversion, and that the CT decay kinetics are connected to the CT state energy via the energy-gap law. Notably, the slope of the exponential factor in Equation 6.2 agrees fairly well with the result from a recent charge recombination study on nonfullerene blends comprising the polymer MEH-PPV and different fluorene-based acceptors. This implies a common mechanism determining the CT decay in organic donor-acceptor blends. Recent theoretical and experimental work suggested that the nonradiative decay of CT states involves high-frequency carbon-carbon stretching modes of the $\pi$-conjugated carbon framework, which are intrinsic to organic semiconducting materials. 


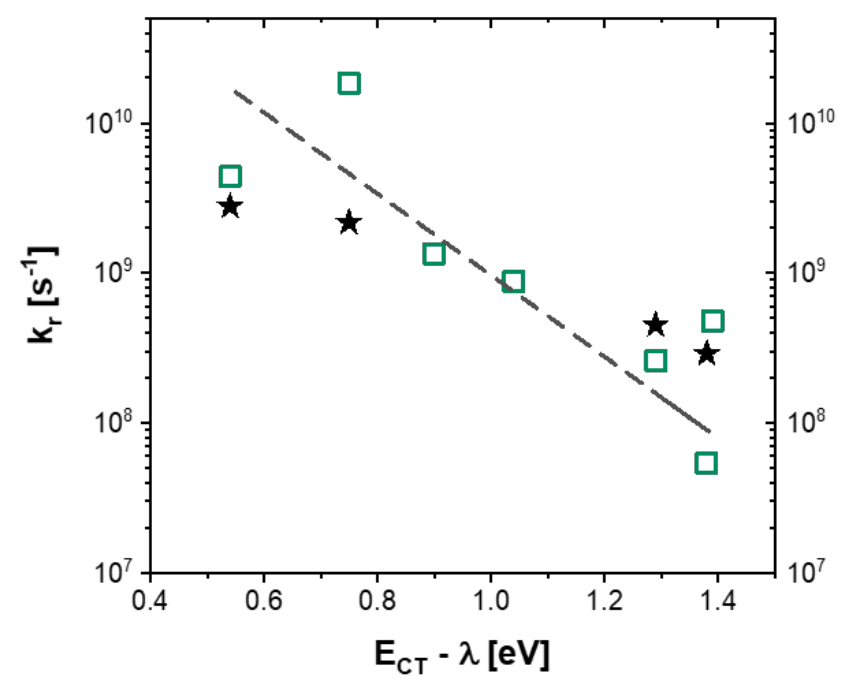

Figure 6.11: $k_{r}$ from the analysis of the TDCF data (open squares) performed by our collaborators and from our fluence dependent TAS data (stars), plotted as function $E_{C T}-$ $\lambda$. The dashed line is calculated from Equation 6.2 with $h v_{v} / B=200 \mathrm{meV}$. Reprinted with permission from [63]. Copyright 2019 American Chemical Society.

\subsection{Conclusions}

In this work we performed TAS measurements, in combination with steadystate and transient techniques from our collaborators, to a subset of four selected low-donor-content blends, TAPC: $\mathrm{C}_{60}$, rubrene: $\mathrm{C}_{60}, \mathrm{~m}-\mathrm{MTDATA}: \mathrm{C}_{60}$, and TPDP: $\mathrm{C}_{60}$, from a larger set of samples which revealed a correlation between the $V_{O C}$ and the quality of the $\mathrm{J}-\mathrm{V}$ characteristics as expressed by the parameter $\mathrm{FF}^{*}$, with photocurrent generation becoming less efficient and more field-dependent when decreasing the CT state energy.

Despite all studied blends having a small concentration of molecular donors dispersed into a $\mathrm{C}_{60}$-rich matrix, some systems display a strong effect of the electric field on photocurrent generation, while others do not. This suggests that 
the aggregation of only one component is not sufficient to guarantee efficient charge collection, and that extensive hole delocalization, e.g., along a conjugated polymer backbone or within molecular aggregate, is not a necessary prerequisite for field-independent photocurrent generation.

Bias-dependent EQE measurements and TDCF measurements from our collaborators using different excitation wavelengths suggest that photocurrent generation involves a low energy donor-acceptor CT state manifold. This is consistent with the minor fluence dependence we measured by TAS on the nanosecond time scale, indicating that a first-order recombination process dominates the fate of the photogenerated carriers at this fluences. We argue that this kinetic scenario stems from the rapid reformation of CT states from free charges through efficient bimolecular recombination, which enables the CT lifetime to be recovered from the observed recombination kinetics.

Our charge carrier dynamics measured by TAS in each blend, was global fit accross different pump fluences using a simple kinetic model to describe the competition between CT dissociation and recombination, including CT reformation through the bimolecular recombination of free charge. CT Decay rates estimated from the global fit exhibit a 10-fold increase when going from rubrene to TPDP. And when these values of $k_{r}$ as well as the values estimated from TDCF via Monte Carlo simulations by our collaborator are plotted as a function of $E_{C T}-\lambda$, both data sets align with the prediction of the energy gap law.

Particularly useful conclusions that we extract from this extensive study, are the following:

- that blends with low-lying CT states (and low $V_{O C}$ ) suffer from high CT 
state decay rates which are determined by nonradiative internal conversion, and,

- that the dynamics of the CT decay are connected to the CT state energy via the energy-gap law.

The main implication of this, is the importance of reducing the rate of CT state decay in future OPV devices. We note that despite the CT states being key for the understanding of charge photo-generation, there is a lack of information on its intrinsic decay, which should not be confused with the CT state lifetime, as is typically deduced from TA or transient photoluminescence, which comprise contributions from all processes depopulating the CT state, including CT state dissociation. Our work on morphologically well-defined low-donorcontent blends proposes a direct energy gap-type link between the efficiency of photocurrent generation and the energy of the relevant CT state.

It is worth noting that in state-of-the-art bulk heterojunction OPVs it has been shown that the microscopic details of the heterojunction, in particular the molecular packing and orientation of the chromophores at the heterojunction and the intermolecular coupling and wave function delocalization affect the CT decay process, rendering the investigation and manipulation of the CT decay kinetics an exciting but also challenging topic of OPV research. Nevertheless, we argue that increasing the $V_{O C}$ by increasing the CT energy in bulk heterojunction blends is likely to equally improve all other photovoltaic parameters. In fact, some novel high $V_{O C}$ blends with nonfullerene acceptors yielded efficient charge generation despite a nearly zero energy offset at the heterojunction $[59,76,167$, $168]$. 


\section{Chapter 7}

\section{Conclusions}

In this thesis work we performed spectroscopic studies of organic photovoltaic (OPV) active layers (without contacts) using transient absorption spectroscopy (TAS). This technique allows us to directly measure the photo-excited species created upon light absorption in order to investigate the mechanisms of charge photo-current generation. We disentangled the contributions from individual transient species to the overall TAS signal via the soft-modelling algorithm known as Multivariate Curve Resolution by Alternating Least Squares (MCR-ALS), and we used a simple kinetic model, which takes into account that CT states mediate charge generation and recombination, to isolate the dynamics of bound CT pair.

In Chapter 4 we investigated a family of related polymers based on PTBT, where the sulphur atom in the thiadiazole unit was substituted with oxygen or selenium in order to investigate how atom substitution affects the photophysics in this family of related low-bandgap copolymers for photovoltaic cells. Our results on the PTBO and PTBSe systems explain the superior photovoltaic performance of the original sulfur-containing variant PTBT, highlighting the low tolerance of these materials to backbone substitutions. In both PTBO and PTBSe 
systems, we identify strong recombination of geminate CT pairs as the major limiting factor of the Jsc and FF photovoltaic parameters. This is attributed to unfavourable electronic and conformational properties at the donor:acceptor interface. In the particular case of PTBSe:PC61BM, the recombination pathway of CT states with triplet character into the triplet exciton manifold is facilitated by the heavy atom effect, in addition to a highly intermixed morphology.

Chapter 5 comprises the spectroscopic comparison between OPV layers featuring the same polymer donor PPDT2FBT matched with three different acceptors, namely the fullerene acceptor PC70BM, the small molecule nonfullerene acceptor NIDCS-HO and the polymeric acceptor N2200. Our results provide insight on the processes that limit the performance of each device, showing that small molecule NFA are promising acceptors, since morphology and disorder, the factors that we have found to be limiting the device performance, could potentially be tuned for the development of more efficient materials. In the fullerene and NIDCS-HO -based devices, we find that hole transfer from acceptor to donor gives rise to a delayed increase of charges in the donor phase. However, the advantage of this contribution to charges that could potentially be extracted in a functional device, is limited by the morphology in the NIDCSHO -based device. For the all-polymer device based on the N2200 acceptor, we find that both geminate and nongeminate recombination are limiting the photovoltaic performance.

Lastly, in chapter 6 we investigated charge carrier dynamics in a series of solar devices composed predominantly of C60 and small amounts of organic small molecule donors, where their CT state energies are systematically varied. Our results, in combination with time-delayed collection field (TDCF), and external quantum efficiency measurements (EQE) measurements at different bias 
performed by our collaborators, allow us to identify geminate recombination as the major loss channel and we find that the dynamics of the CT decay are connected to the CT state energy via the energy-gap law. In this way, the energy of the CT state is identified as the main parameter determining the efficiency of photocurrent generation in these morphologically well-defined donor:acceptor blends.

Overall, the contributions in this thesis work demonstrate how TAS measurements can provide valuable information to construct a comprehensive picture of the underpinning mechanisms of charge photo-current generation in OPV layers. In terms of modelling, we have shown that a kinetic model where CT states are explicitly mediating charge generation and recombination can successfully explain TAS fluence-dependent data in different systems, including fullerene and nonfullerene OPV blend films. 


\section{Appendix A}

\section{Sample Preparation}

As mentioned in Section 3.1 of this thesis, the materials PTBO, PTBSe, PPDT2FBT and NIDCS-HO studied in Chapters 4 and 5 were provided by our collaborators from Korea University in the research group of Prof. H. Y. Woo. The samples studied in Chapter 6 were fabricated by our collaborators from University of Postdam in the research group of Prof. Dieter Neher.

\section{A.1 Preparation of the PTBO and PTBSe solutions}

Casting solutions of PTBO, PTBSe and their fullerene blends were prepared following the preparation conditions of the PTBT parent system [87]. Chlorobenzene (CB) was used as the solvent, and solutions were prepared with a concentration of $11.1 \mathrm{mg} / \mathrm{ml}$ in the case of neat polymer, and a total polymer+fullerene (donor+acceptor) concentration $22.2 \mathrm{mg} / \mathrm{ml}$ in the case of the blends. In the blend solutions the donor:acceptor ratio was $1: 2(\mathrm{w} / \mathrm{w})$. Films were spin coated on quartz substrates at $1500 \mathrm{rpm}$ for $60 \mathrm{~s}$. 


\section{A.2 Preparation of the PPDT2FBT:PC 70 BM solution}

This solution was made such that the polymer:fullerene (donor:acceptor) ratio was $1: 1.5(\mathrm{w} / \mathrm{w})$ and the total concentration of donor+acceptor in chlorobenzene (CB) solution was $15 \mathrm{mg} / \mathrm{ml}$. A volume fraction of 2 vol\% of additive diphenlyether (DPE) was used. The solution was stirred at $80^{\circ} \mathrm{C}$ overnight before being spin-coated at $1000 \mathrm{rpm}$ for $40 \mathrm{~s}$ onto a quartz substrate. Post thermal annealing treatment was performed at $70^{\circ} \mathrm{C}$ during $20 \mathrm{~min}$ on a heating plate.

\section{A.3 Preparation of the PPDT2FBT:NIDCS-HO solu- tion}

This solution consisted on a donor:acceptor ratio of $1: 2.5(\mathrm{w} / \mathrm{w})$ and the total concentration of donor+acceptor in Chloroform (CF) solution was $10 \mathrm{mg} / \mathrm{ml}$. The solution was stirred at $55^{\circ} \mathrm{C}$ overnight before being spin-coated at $1000 \mathrm{rpm}$ for $60 \mathrm{~s}$ onto a quartz substrate. Post thermal annealing was performed at $90^{\circ} \mathrm{C}$ during $10 \mathrm{~min}$ on a heating plate.

\section{A.4 Preparation of PPDT2FBT:N2200 solution}

For this blend, the donor:acceptor ratio was $1: 0.7(\mathrm{w} / \mathrm{w})$ and the total concentration of donor+acceptor in Chloroform (CF) solution $10 \mathrm{mg} / \mathrm{ml}$. The volume fraction of diphenlyether (DPE) additive to donor+acceptor in the CF solution was 1 vol\%. The solution was stirred at $45^{\circ} \mathrm{C}$ for one day before being spin-coated onto a quartz substrate. 


\section{Bibliography}

[1] The Causes of Climate Change. Accessed 25 July 2020, https://climate.nasa.gov/causes/

[2] Fossil fuels, explained. Accessed 25 July 2020, https://www.nationalgeographic.com/environment/energy/reference/fossilfuels/

[3] Climate Change: How Do We Know?. Accessed 25 July 2020, https://climate.nasa.gov/evidence/

[4] What is the Paris Agreement? Accessed 25 July 2020, https://unfccc.int/process-and-meetings/the-paris-agreement/whatis-the-paris-agreement

[5] Special Report: Global Warming of $1.5^{\mathrm{O}}$ C. Accessed 25 July 2020, https://www.ipcc.ch/sr15/

[6] A Degree of Concern: Why Global Temperatures Matter. Accessed 25 July 2020, https://climate.nasa.gov/news/2878/a-degree-of-concern-why-globaltemperatures-matter/

[7] Pathways for solar photovoltaics, Joel Jean, Patrick R. Brown, Robert L. Jaffe, Tonio Buonassisi and Vladimir Bulović. Energy Environ. Sci., 2015, 8, 1200. 
[8] A Fundamental Look At Supply Side Energy Reserves For The Planet. Accessed 25 July 2020, https://www.iea-shc.org/data/sites/1/publications/201511-A-Fundamental-Look-at-Supply-Side-Energy-Reserves-for-thePlanet.pdf

[9] Best Research-Cell Efficiency Chart. Accessed 25 July 2020, https://www.nrel.gov/pv/cell-efficiency.html

[10] Solar Energy. Accessed 25 July 2020, https://www.irena.org/solar

[11] Roll-to-roll fabrication of polymer solar cells. Roar Søndergaard, Markus Hösel, Dechan Angmo, Thue T.Larsen-Olsen and Frederik C.Krebs. Materials Today Volume 15, Issues 1-2, Pages 36-49.

[12] Wide-gap non-fullerene acceptor enabling high-performance organic photovoltaic cells for indoor applications. Yong Cui, Yuming Wang, Jonas Bergqvist, Huifeng Yao, Ye Xu, Bowei Gao, Chenyi Yang, Shaoqing Zhang, Olle Inganäs, Feng Gao and Jianhui Hou. Nature Energy, volume 4, pages 768-775 (2019).

[13] Electronic Processes in Organic Semiconductors. Anna Köhler and Heinz Bässler. ISBN: 978-3-527-33292-2.

[14] Principles of Molecular Photochemistry, Nicholas J. Turro, V. Ramamurthy and J.C. Scaiano, University Science Books (2009), ISBN 978-1-891389-57-3.

[15] Synthesis of electrically conducting organic polymers: halogen derivatives of polyacetylene, (CH)x. Hideki Shirakawa, Edwin J. Louis, Alan G. MacDiarmid, Chwan K. Chiang and Alan J. Heeger. J. Chem. Soc., Chem. Commun., 1977, 578-580. 
[16] The Nobel Prize in Chemistry 2000, accessed 31 March 2020, https://www.nobelprize.org/prizes/chemistry/2000/summary/

[17] Franck-Condon Principle, Govindjee, based on E.Rabinowitch and Govindjee, 1969. Accessed 31 March 2020, http://www.life.illinois.edu/govindjee/biochem494/Abs.html

[18] What is the Binding Energy of a Charge Transfer State in an Organic Solar Cell? Stavros Athanasopoulos, Franz Schauer, Vojtech Nádaždy, Mareike Weiß, Frank-Julian Kahle, Ullrich Scherf, Heinz Bässler and Anna Köhler. Advanced Energy Materials, Volume 9, Issue 24, 2019.

[19] Elementary processes of photochemical reactions, J. Frank, Transactions of the Faraday Society 21, 536-542 (1926).

[20] A theory of intensity distribution in band systems, E. Condon, Physical Review 28, 1182-1201 (1926).

[21] Temas de Mecánica Cuántica. Raul Oscar Barrachina. 1993.

[22] Electronic Spectra of Molecules, accessed 31 March 2020, http://hyperphysics.phy-astr.gsu.edu/hbase/molecule/molelect.html

[23] Measurements of exciton diffusion in conjugated polymers. Paul Edward Shaw. Dissertation.

[24] Molecular Doping Processes in Organic Semiconductors investigated by Photoelectron Spectroscopy. Max L. Tietze. Dissertation.

[25] Franck-Condon principle, Wikipedia Image.

[26] Radiationless Transitions in Polyatomic Molecules. I. Calculation of FranckCondon Factors. W. Siebrand, J. Chem. Phys. 46, 440 (1967). 
[27] Theory of electronic energy relaxation in solid phase. Robinson, G.W. and Frosch, R.P., J. Chem. Phys., 37 (9), 1962.

[28] The Nobel Prize in Chemistry 1992, accessed 11 August 2020, https://www.nobelprize.org/prizes/chemistry/1992/summary/

[29] Charge Photogeneration in Organic Solar Cells, Tracey M. Clarke and James R. Durrant, Chem. Rev. 2010, 110, 6736-6767.

[30] Two-layer organic photovoltaic cell. C. W. Tang. Appl. Phys. Lett. 48, 183 (1986).

[31] Semiconducting polymer-buckminsterfullerene heterojunctions: Diodes, photodiodes, and photovoltaic cells, N. S. Sariciftci, D. Braun, and C. Zhang, Appl. Phys. Lett. 62, 585 (1993).

[32] Polymer Photovoltaic Cells: Enhanced Efficiencies via a Network of Internal Donor-Acceptor Heterojunctions, G. Yu, J. Gao, J. C. Hummelen, F. Wudi, A. J. Heeger, Science 1995, 270, 1789.

[33] Preparation and Characterization of Fulleroid and Methanofullerene Derivatives. Jan C. Hummelen, Brian W. Knight, F. LePeq, Fred Wudl, Jie Yao and Charles L. Wilkins. J. Org. Chem. 1995, 60, 3, 532-538.

[34] Fullerenes for Bioscience and Photovoltaic Applications, Michael D. Diener, accessed 31 March 2020, https://www.sigmaaldrich.com/technicaldocuments/articles/materials-science/nanomaterials/fullerenes.html

[35] Organic solar cells based on non-fullerene acceptors. Jianhui Hou, Olle Inganäs, Richard H. Friend and Feng Gao. Nature Materials, volume 17, 119128(2018). 
[36] Low band gap polymers for organic photovoltaics. Eva Bundgaard and Frederik C. Krebs. Solar Energy Materials \& Solar Cells 91 (2007) 954-985.

[37] High Photovoltaic Performance of a Low-Bandgap Polymer. D. Mühlbacher M. Scharber M. Morana Z. Zhu D. Waller R. Gaudiana C. Brabec. Adv Mater (2006) 18(21), 2884.

[38] Regioregular poly(3-hexyl)selenophene: a low band gap organic hole transporting polymer. Martin Heeney, Weimin Zhang, David J. Crouch, Michael L. Chabinyc, Sergey Gordeyev, Rick Hamilton, Simon J. Higgins, Iain McCulloch, Peter J. Skabara, David Sparrowea and Steve Tierney. Chem. Commun., 2007, 5061-5063.

[39] Alternate donor-acceptor small-band-gap semiconducting polymers; Polysquaraines and polycroconaines. E. E. Havinga, W.ten Hoeve and H. Wynberg. Synth. Met. 1993, 55, 299-306.

[40] Atomistic Band Gap Engineering in Donor-Acceptor Polymers. Gregory L. Gibson, Theresa M. McCormick and Dwight S. Seferos. J. Am. Chem.Soc. 2012, $134,539-547$.

[41] Polymer:fullerene bulk heterojunction solar cells. Jenny Nelson. Materials Today, Volume 14, Issue 10, October 2011, Pages 462-470.

[42] High-efficiency photovoltaic devices based on annealed poly(3hexylthiophene) and 1-(3-methoxycarbonyl)-propyl-1-phenyl-(6,6)c-61 blends. M. Reyes-Reyes, K. Kim, and D. L. Carroll. Applied Physics Letters 87, 083506 (2005).

[43] Aggregation and morphology control enables multiple cases of highefficiency polymer solar cells. Yuhang Liu, Jingbo Zhao, Zhengke Li, Cheng 
Mu, Wei Ma, Huawei Hu, Kui Jiang, Haoran Lin, Harald Ade and He Yan. Nature Communications volume 5, Article number: 5293 (2014).

[44] A low cost and high performance polymer donor material for polymer solar cells. Chenkai Sun, Fei Pan, Haijun Bin, Jianqi Zhang, Lingwei Xue, Beibei Qiu, Zhixiang Wei, Zhi-Guo Zhang and Yongfang Li. Nature Communications volume 9, Article number: 743 (2018).

[45] Semi-crystalline photovoltaic polymers with efficiency exceeding $9 \%$ in a $300 \mathrm{~nm}$ thick conventional single-cell device, T. L. Nguyen, H. Choi, S.-J. Ko, M. A. Uddin, B. Walker, S. Yum, J.-E. Jeong, M. H. Yun, T. J. Shin, S. Hwang, J. Y. Kim and H. Y. Woo, Energy Environ. Sci., 2014, 7, 3040.

[46] Determining the Role of Polymer Molecular Weight for High-Performance All-Polymer Solar Cells: Its Effect on Polymer Aggregation and Phase Separation, Hyunbum Kang, Mohammad Afsar Uddin, Changyeon Lee, Ki-Hyun Kim, Thanh Luan Nguyen, Wonho Lee, Yuxiang Li, Cheng Wang, Han Young Woo and Bumjoon J. Kim, J. Am. Chem. Soc. 2015, 137, 2359-2365.

[47] A High Efficiency Nonfullerene Organic Solar Cell with Optimized Crystalline Organizations, Oh Kyu Kwon, Mohammad Afsar Uddin, Jung-Hwa Park, Sang Kyu Park, Thanh Luan Nguyen, Han Young Woo and Soo Young Park, Adv. Mater. 2016, 28, 910-916.

[48] Low-Bandgap Donor/Acceptor Polymer Blend Solar Cells with Efficiency Exceeding 4\%. Daisuke Mori , Hiroaki Benten, Izumi Okada, Hideo Ohkita, and Shinzaburo Ito. Adv. Energy Mater. 2014, 4, 1301006.

[49] Light Harvesting for Organic Photovoltaics Gordon J. Hedley, Arvydas Ruseckas, and Ifor D. W. Samuel. Chem Rev. 2017 Jan 25; 117(2): 796-837. 
[50] Charge Carrier Generation, Recombination, and Extraction in PolymerFullerene Bulk Heterojunction Organic Solar Cells. Frédéric Laquai, Denis Andrienko, Carsten Deibel, and Dieter Neher. Elementary Processes in Organic Photovoltaics pp 267-291.

[51] Role of the Charge Transfer State in Organic Donor-Acceptor Solar Cells. Carsten Deibel and Thomas Strobel Vladimir Dyakonov. Advanced Materials. Volume 22, Issue 37, 2010, Pages 4097-4111.

[52] Understanding Energy Loss in Organic Solar Cells: Toward a New Efficiency Regime. S. Matthew Menke, Niva A. Ran, Guillermo C. Bazan, and Richard H. Friend. Joule, Volume 2, Issue 1, 17 January 2018, Pages 25-35.

[53] Spectroscopic investigation of excitonic and charge photogeneration processes in organic photovoltaic cells, Joseph Keith Gallaher, 2016. Dissertation.

[54] The role of driving energy and delocalized states for charge separation in organic semiconductors. Bakulin AA, Rao A, Pavelyev VG, van Loosdrecht PH, PshenichnikovMS, et al. Science 335:1340-44, 2012.

[55] Ultrafast charge separation in organic photovoltaics enhanced by charge delocalization and vibronically hot exciton dissociation. Tamura H, Burghardt I. J. Am. Chem. Soc. 135:16364-67, 2013.

[56] Charge Separation Pathways in a Highly Efficient Polymer: Fullerene Solar Cell Material. Arun Aby Paraecattil, Natalie Banerji. J. Am. Chem. Soc. 2014, $136,4,1472-1482$.

[57] Dissociation of Charge Transfer States and Carrier Separation in Bilayer Organic Solar Cells: A Time-Resolved Electroabsorption Spectroscopy Study. Andrius Devižis, Jelissa De Jonghe-Risse, Roland Hany, Frank Nüesch, Sandra 
Jenatsch, Vidmantas Gulbinas, Jacques-E. Moser. J. Am. Chem. Soc. 2015, 137, 25, 8192-819.

[58] Efficient charge generation by relaxed charge-transfer states at organic interfaces. Koen Vandewal, Steve Albrecht, Eric T. Hoke, Kenneth R. Graham, JohannesWidmer, Jessica D. Douglas, Marcel Schubert, William R. Mateker, Jason T. Bloking, George F. Burkhard, Alan Sellinger, Jean M. J. Fréchet, Aram Amassian, Moritz K. Riede, Michael D. McGehee, Dieter Neher and Alberto Salleo. Nature Materials volume 13, pages63-68(2014).

[59] Fast charge separation in a non-fullerene organic solar cell with a small driving force. Jing Liu, Shangshang Chen, Deping Qian, Bhoj Gautam, Guofang Yang, Jingbo Zhao, Jonas Bergqvist, Fengling Zhang, Wei Ma, Harald Ade, Olle Inganäs, Kenan Gundogdu, Feng Gao and He Yan. Nature Energy volume 1, Article number: 16089 (2016).

[60] Understanding the High Performance of over 15\% Efficiency in SingleJunction Bulk Heterojunction Organic Solar Cells. Akchheta Karki, Joachim Vollbrecht, Alana L. Dixon, Nora Schopp, Max Schrock, G. N. Manjunatha Reddy, and Thuc-Quyen Nguyen. Adv. Mater. 2019, 31, 1903868.

[61] Effect of Morphology on Ultrafast Free Carrier Generation in Polythiophene:Fullerene Organic Solar Cells, Ian A. Howard, Ralf Mauer, Michael Meister, and Frédéric Laquai, J. Am. Chem. Soc. 2010, 132, 14866-14876.

[62] Thermal Annealing Reduces Geminate Recombination in TQ1:N2200 AllPolymer Solar Cells, Safakath Karuthedath, Armantas Melianas, Zhipeng Kan, Vytenis Pranculis, Markus Wohlfahrt, Jafar I. Khan, Julien Gorenflot, Yuxin Xia, Olle Inganäs, Vidmantas Gulbinas, Martijn Kemerink, and Frédéric Laquai, J. Mater. Chem. A, 2018,6, 7428-7438. 
[63] Energy-Gap Law for Photocurrent Generation in Fullerene-Based Organic Solar Cells: The Case of Low-Donor-Content Blends. Elisa Collado-Fregoso, Silvina N. Pugliese, Mariusz Wojcik, Johannes Benduhn, Eyal Bar-Or, Lorena Perdigón Toro, Ulrich Hörmann, Donato Spoltore, Koen Vandewal, Justin M. Hodgkiss and Dieter Neher. J. Am. Chem. Soc. 2019, 141, 6, 2329-2341.

[64] The Effect of Solvent Additives on Morphology and Excited-State Dynamics in PCPDTBT:PCBM Photovoltaic Blends. Fabian Etzold, Ian A. Howard, Nina Forler, Don M. Cho, Michael Meister, Hannah Mangold, Jie Shu, Michael Ryan Hansen, Klaus Müllen, and Frédéric Laquai. J. Am. Chem. Soc. 2012, 134, 10569-10583.

[65] The Role of Mobility on Charge Generation, Recombination and Extraction in Polymer-Based Solar Cells. Safa Shoaee, Martin Stolterfoht and Dieter Neher. Adv. Energy Mater. 2018, 1703355.

[66] Models of charge pair generation in organic solar cells. Sheridan Few, Jarvist M. Frost and Jenny Nelson. Phys. Chem. Chem. Phys., 2015, 17, 2311.

[67] Physics of Solar Cells, J. Nelson, Imperial College Press, London (2003).

[68] Intrinsic Non-Radiative Voltage Losses in Fullerene-Based Organic Solar Cells. Benduhn, J.; Tvingstedt, K.; Piersimoni, F.; Ullbrich, S.; Fan, Y.; Tropiano, M.; McGarry, K. A.; Zeika, O.; Riede, M. K.; Douglas, C. J.; Barlow, S.; Marder, S. R.; Neher, D.; Spoltore, D.; Vandewal, K. Nat. Energy 2017, 2, 17053.

[69] A New Figure of Merit for Organic Solar Cells with Transport-limited Photocurrents. Dieter Neher, Juliane Kniepert, Arik Elimelech \& L. Jan Anton Koster. Scientific Reports volume 6, Article number: 24861 (2016).

[70] Exciton Diffusion in Conjugated Polymers: From Fundamental Understanding to Improvement in Photovoltaic Conversion Efficiency. Yasunari Tamai, 
Hideo Ohkita, Hiroaki Benten, Shinzaburo Ito. J. Phys. Chem. Lett. 2015, 6, 17, 3417-3428.

[71] Exciton diffusion in organic semiconductors. Mikhnenko, O.V.; Blom, P.W.M.; Nguyen, T.-Q. Energy Environ. Sci. 2015, 8, 1867-1888.

[72] Singlet Exciton Lifetimes in Conjugated Polymer Films for Organic Solar Cells. Stoichko D. Dimitrov, Bob C. Schroeder, Christian B. Nielsen, Hugo Bronstein, Zhuping Fei 1, Iain McCulloch, Martin Heeney and James R. Durrant. Polymers 2016, 8, 14.

[73] The energy gap law for radiationless transitions in large molecules. Englman, R. and Jortner, J. Mol. Phys. 18, 145-164 (1970).

[74] On the relation between radiative and nonradiative transitions in molecules. Siebrand, W. Chem. Phys. Lett. 1971, 9, 157-159.

[75] High Exciton Diffusion Coefficients in Fused Ring Electron Acceptor Films. Sreelakshmi Chandrabose, Kai Chen, Alex J. Barker, Joshua J. Sutton, Shyamal K. K. Prasad, Jingshuai Zhu, Jiadong Zhou, Keith C. Gordon, Zengqi Xie, Xiaowei Zhan, and Justin M. Hodgkiss. J. Am. Chem. Soc. 2019, 141, 6922-6929.

[76] Impact of interfacial molecular orientation on radiative recombination and charge generation efficiency. Niva A. Ran, Steffen Roland, John A. Love, Victoria Savikhin, Christopher J. Takacs, Yao-Tsung Fu, Hong Li, Veaceslav Coropceanu, Xiaofeng Liu, Jean-Luc Brédas, Guillermo C. Bazan, Michael F. Toney, Dieter Neher and Thuc-Quyen Nguyen. Nat Commun. 2017 Jul 19;8(1):79.

[77] Barrierless Free Charge Generation in the High-Performance PM6:Y6 Bulk Heterojunction Non-Fullerene Solar Cell. Lorena Perdigón-Toro, Huotian 
Zhang, Anastasia Markina, Jun Yuan, Seyed Mehrdad Hosseini, Christian M. Wolff, Guangzheng Zuo, Martin Stolterfoht, Yingping Zou, Feng Gao, Denis Andrienko, Safa Shoaee and Dieter Neher. Volume 32, Issue 9, 2020, 1906763.

[78] Suppression of geminate charge recombination in organic photovoltaic devices with a cascaded energy heterojunction. Chris Groves. Energy Environ. Sci., 2013,6, 1546-1551.

[79] Bimolecular recombination in polymer/ fullerene bulk heterojunction solar cells. L. J. A. Koster, V. D. Mihailetchi, and P. W. M. Blom. Phys. Lett. 88, 052104 (2006).

[80] Effect of traps on the performance of bulk heterojunction organic solar cells. M. M. Mandoc, F. B. Kooistra, J. C. Hummelen, B. de Boer, and P. W. M. Blom. Appl. Phys. Lett. 91, 263505 (2007).

[81] Overcoming Geminate Recombination and Enhancing Extraction in Solution-Processed Small Molecule Solar Cells. Christopher M. Proctor , Steve Albrecht, Martijn Kuik, Dieter Neher , and Thuc-Quyen Nguyen. Advanced Energy Materials. Volume 4, Issue 10, 2014, 1400230.

[82] Charge Generation and Recombination in Organic Photovoltaics, Michelle Southworth Vezie. 2017. Dissertation.

[83] J-Aggregates: From Serendipitous Discovery to Supramolecular Engineering of Functional Dye Materials. Frank Würthner, Theo E. Kaiser, and Chantu R. Saha-Möller. Angew. Chem. Int. Ed. 2011, 50, 3376-3410.

[84] El laser, la luz de nuestro tiempo. Fernandez Benjamin Alonso et al.

[85] Investigation of water ionization by interaction with laser radiation. Silvina N. Pugliese, Master Thesis. 
[86] Distance Distributions of Photogenerated Charge Pairs in Organic Photovoltaic Cells. Barker, A. J.; Chen, K.; Hodgkiss, J. M. J. Am. Chem. Soc. 2014, 136, 12018-12026.

[87] Spectroscopically Tracking Charge Separation in Polymer : Fullerene Blends with a Three-Phase Morphology. Gallaher, J. K.; Prasad, S. K. K.; Uddin, M. A.; Kim, T.; Kim, J. Y.; Woo, H. Y.; Hodgkiss, J. M. Energy Environ. Sci. 2015, 8, 2713-2724.

[88] Sub-50 fs broadband absorption spectroscopy with tunable excitation: putting the analysis of ultrafast molecular dynamics on solid ground. U. Megerle, I. Pugliesi, C. Schriever, C.F. Sailer and E. Riedle. Appl Phys B (2009) 96: $215-231$.

[89] Unraveling Efficiency-Limiting Processes in Organic Solar Cells by Ultrafast Spectroscopy - Impact of Chemical Structure and Morphology on Photophysics and Efficiency. Dominik Werner Gehrig. Dissertation.

[90] Evolving Factor Analysis for the Resolution of Overlapping Chromatographic Peaks. Marcel Maeder and Arne Zilian. Chemometrics and Intelligent Laboratory Systems. Volume 3, Issue 3, March 1988, Pages 205-213.

[91] A graphical user-friendly interface for MCR-ALS: a new tool for multivariate curve resolution in MATLAB. Joaquim Jaumot, Raimundo Gargallo, Anna de Juan, Romà Tauler. Chemometrics and Intelligent Laboratory Systems 76 (2005) 101-110.

[92] Multivariate Curve Resolution (MCR). Solving the mixture analysis problem. Anna de Juan, Joaquim Jaumot and Romà Tauler. Anal. Methods, 2014, 6, 4964. 
[93] Multivariate curve resolution: A review of advanced and tailored applications and challenges, C. Ruckebusch and L. Blanchet, Analytica Chimica Acta 765 (2013) 28-36.

[94] Transient absorption data analysis by soft-modelling. I.A. Howard, H. Mangold, F. Etzold, D. Gehrig, and F. Laquai.

[95] Sub-ns triplet state formation by non-geminate recombination in PSBTBT:PC70BM and PCPDTBT:PC60BM organic solar cells. Fabian Etzold, Ian A. Howard, Nina Forler, Anton Melnyk, Denis Andrienko, Michael Ryan Hansen and Frédéric Laquai. Energy Environ. Sci., 2015, 8, 1511-1522.

[96] Triplet state formation in photovoltaic blends of DPP-type copolymers and PC71BM. Ochsmann JR, Chandran D, Gehrig DW, Anwar H, Madathil PK, Lee KS, Laquai F. Macromol. Rapid Commun. 2015, 36, 1122

[97] High open-circuit voltage small-molecule p-DTS(FBTTh2)2:ICBA bulk heterojunction solar cells - morphology, excited-state dynamics, and photovoltaic performance. Aung Ko Ko Kyaw, Dominik Gehrig, Jie Zhang, Ye Huang, Guillermo C. Bazan, Frédéric Laquai and Thuc-Quyen Nguyen. J. Mater. Chem. A, 2015,3, 1530-1539.

[98] From Recombination Dynamics to Device Performance: Quantifying the Efficiency of Exciton Dissociation, Charge Separation, and Extraction in Bulk Heterojunction Solar Cells with Fluorine-Substituted Polymer Donors. Julien Gorenflot, Andreas Paulke, Fortunato Piersimoni, Jannic Wolf, Zhipeng Kan, Federico Cruciani, Abdulrahman El Labban, Dieter Neher, Pierre M. Beaujuge, and Frédéric Laquai. Adv. Energy Mater. 2017, 1701678. 
[99] Effect of Group-14 and Group-16 Substitution on the Photophysics of Structurally Related Donor-Acceptor Polymers. Gregory L. Gibson, Theresa M. McCormick and Dwight S. Seferos. J. Phys. Chem. C 2013, 117, 16606-16615.

[100] Effects of heteroatom substitution in conjugated heterocyclic compounds on photovoltaic performance: from sulfur to tellurium. Y. S. Park, T. S. Kale, C.-Y. Nam, D. Choib and R. B. Grubbs. Chem. Commun., 2014, 50, 7964.

[101] Donor-Acceptor Small Molecules for Organic Photovoltaics: Single-Atom Substitution (Se or S). Xiaoming He, Bing Cao, Tate C. Hauger, Minkyu Kang, Sergey Gusarov, Erik J. Luber and Jillian M. Buriak. ACS Appl. Mater. Interfaces 2015, 7, 8188-8199.

[102] Systematic control of heteroatoms in donor-acceptor copolymers and its effects on molecular conformation and photovoltaic performance. Sebastian Wood, Ji-Hoon Kim, Jessica Wade, Jong Baek Park, Do-Hoon Hwang and Ji-Seon Kim. J. Mater. Chem. C, 2016,4, 7966-7978.

[103] Selenium-substituted polymers for improved photovoltaic performance. Jiangsheng Yu, Guanqun Ding, Jiefeng Hai, Enwei Zhu, Xinxing Yin, Zhongsheng Xu, Baojing Zhou, Fujun Zhang, Wanli Ma and Weihua Tang. Phys. Chem. Chem. Phys., 2016,18, 7978-7986.

[104] The role of spin in the kinetic control of recombination in organic photovoltaics. Akshay Rao, Philip C. Y. Chow, Simon Gélinas, Cody W. Schlenker, Chang-Zhi Li, Hin-Lap Yip, Alex K.-Y. Jen, David S. Ginger and Richard H. Friend. Nature volume 500, 435-439 (2013).

[105] Quantitative Bimolecular Recombination in Organic Photovoltaics through Triplet Exciton Formation. Philip C. Y. Chow, Simon Gélinas, Akshay Rao, and Richard H. Friend. J. Am. Chem. Soc. 2014, 136, 3424-3429. 
[106] Polaron pair mediated triplet generation in polymer/fullerene blends. Stoichko D. Dimitrov, Scot Wheeler, Dorota Niedzialek, Bob C. Schroeder, Hendrik Utzat, Jarvist M. Frost, Jizhong Yao, Alexander Gillett, Pabitra S. Tuladhar, Iain McCulloch, Jenny Nelson and James R. Durrant. Nature Communications volume 6, Article number: 6501 (2015) .

[107] Analysis of Triplet Exciton Loss Pathways in PTB7:PC71BM Bulk Heterojunction Solar Cells. Hannes Kraus, Michael C. Heiber, Stefan Vath, Julia Kern, Carsten Deibel, Andreas Sperlich and Vladimir Dyakonov. Scientific Reports volume 6, Article number: 29158 (2016).

[108] Interplay Between Triplet-, Singlet-Charge Transfer States and Free Charge Carriers Defining Bimolecular Recombination Rate Constant of Organic Solar Cells. Ardalan Armin, James R. Durrant, and Safa Shoaee. J. Phys. Chem. C 2017, 121, 13969-13976.

[109] Decoding Charge Recombination through Charge Generation in Organic Solar Cells. Safa Shoaee, Ardalan Armin, Martin Stolterfoht, Seyed Mehrdad Hosseini, Jona Kurpiers and Dieter Neher. Sol. RRL 2019, 3, 1900184.

[110] Interfacial Charge Transfer States in Condensed Phase Systems. Koen Vandewal. Annu. Rev. Phys. Chem. 2016. 67:113-33.

[111] H- and J-Aggregate Behavior in Polymeric Semiconductors. Frank C. Spano and Carlos Silva. Annu. Rev. Phys. Chem. 2014. 65:477-500.

[112] The Effect of H- and J-Aggregation on the Photophysical and Photovoltaic Properties of Small Thiophene-Pyridine-DPP Molecules for BulkHeterojunction Solar Cells. Miriam Más-Montoya and René A. J. Janssen. Adv. Funct. Mater. 2017, 27, 1605779. 
[113] Subnanosecond Geminate Charge Recombination in Polymer-Polymer Photovoltaic Devices. Justin M. Hodgkiss, Andrew R. Campbell, R. Alex Marsh, Akshay Rao, Sebastian Albert-Seifried, and Richard H. Friend. PRL 104, 177701 (2010).

[114] Performance, morphology and photophysics of high open-circuit voltage, low band gap all-polymer solar cells. Kedar D. Deshmukh, Tianshi Qin, Joseph K. Gallaher, Amelia C. Y. Liu, Eliot Gann, Kane O’Donnell, Lars Thomsen, Justin M. Hodgkiss, Scott E. Watkins and Christopher R. McNeill. Energy Environ. Sci., 2015, 8, 332-342.

[115] Transient Absorption Studies of Bimolecular Recombination Dynamics in Polythiophene/ Fullerene Blend Films. Tracey M. Clarke, Fiona C. Jamieson, and James R. Durrant. J. Phys. Chem. C 2009, 113, 20934-20941.

[116] Ultrafast Exciton Dissociation Followed by Nongeminate Charge Recombination in PCDTBT:PCBM Photovoltaic Blends. Fabian Etzold, Ian A. Howard, Ralf Mauer, Michael Meister, Tae-Dong Kim, Kwang-Sup Lee, Nam Seob Baek, and Frédéric Laquai. J. Am. Chem. Soc. 2011, 133, 9469-9479.

[117] Exciton-Charge Annihilation in Organic Semiconductor Films. Justin M. Hodgkiss Sebastian Albert-Seifried Akshay Rao Alex J. Barker Andrew R. Campbell R. Alex Marsh Richard H. Friend. Advanced Functional Materials. Volume 22, Issue 8, Pages 1567-1577.

[118] Probing the pathways of free charge generation in organic bulk heterojunction solar cells. Jona Kurpiers, Thomas Ferron, Steffen Roland, Marius Jakoby, Tobias Thiede, Frank Jaiser, Steve Albrecht, Silvia Janietz, Brian A. Collins, Ian A. Howard and Dieter Neher. Nature Communications volume 9, Article number: 2038 (2018). 
[119] Ultrafast dynamics of charge carrier photogeneration and geminate recombination in conjugated polymer:fullerene solar cells. Physical Review B 72, 195208, 2005.

[120] Suppression of geminate charge recombination in organic photovoltaic devices with a cascaded energy heterojunction. Chris Groves. Energy Environ. Sci., 2013,6, 1546-1551.

[121] Charge Photogeneration in Neat Conjugated Polymers. Obadiah G. Reid, Ryan D. Pensack, Yin Song, Gregory D. Scholes, and Garry Rumbles. Chem. Mater. 2014, 26, 561-575.

[122] Triplet excitons: improving exciton diffusion length for enhanced organic photovoltaics. Bruno T. Luppi, Darren Majak, Manisha Gupta, Eric Rivard and Karthik Shankar. J. Mater. Chem. A, 2019,7, 2445-2463.

[123] Triplet states in organic semiconductors. A. Köhler and H. Bässler. Materials Science and Engineering R 66 (2009) 71-109.

[124] Sensitized phosphorescence in organic solutions at low temperature. Energy transfer between triplet states. A. Terenin and V. Ermolaev. Trans. Faraday Soc., 1956,52, 1042-1052.

[125] Evidence for the Rapid Conversion of Primary Photoexcitations to Triplet States in Seleno- and Telluro- Analogues of Poly(3-hexylthiophene). Ryan D. Pensack, Yin Song, Theresa M. McCormick, Ashlee A. Jahnke, Jon Hollinger, Dwight S. Seferos and Gregory D. Scholes. J. Phys. Chem. B 2014, 118, 9, 2589-2597.

[126] Charge Carrier Generation Followed by Triplet State Formation, Annihilation, and Carrier Recreation in PBDTTT-C/PC60BM Photovoltaic Blends. 
Dominik W. Gehrig, Ian A. Howard, and Frédéric Laquai. J. Phys. Chem. C 2015, 119, 13509-13515.

[127] Enhanced triplet exciton generation in polyfluorene blends. T. A. Ford, I. Avilov, D. Beljonne, and N. C. Greenham. Physical Review B 71, 125212 (2005).

[128] Photophysical Properties of a 1,2,3,4,5,6-Hexasubstituted Fullerene Derivative. Khin K. China, Shih-Ching Chuanga, Billy Hernandezb, Matthias Selkeb, Christopher S. Footea and Miguel A. Garcia-Garibaya. J Phys Chem A. 2006 December 28; 110(51): 13662-13666.

[129] Plastic Solar Cells. Christoph J. Brabec, N. Serdar Sariciftci and Jan C. Hummelen. Adv. Funct. Mater. 2001, 11, No. 1.

[130] Highly Thermal Stable and Efficient Organic Photovoltaic Cells with Crosslinked Networks Appending Open-Cage Fullerenes as Additives, ChihPing Chen, Chien-Yu Huang and Shih-Ching Chuang, Adv. Funct. Mater. 2015, 25, 207-213.

[131] 18 \% Efficiency organic solar cells. Qishi Liu, Yufan Jiang, Ke Jin, Jianqiang Qin, Jingui Xu, Wenting Li, Ji Xiong,Jinfeng Liu, Zuo Xiao, Kuan Sun, Shangfeng Yang, Xiaotao Zhang, LimingDing. Science Bulletin (2020).

[132] Enhanced Efficiency and Long-Term Stability of Perovskite Solar Cells by Synergistic Effect of Nonhygroscopic Doping in Conjugated Polymer-Based Hole-Transporting Layer, Chang Woo Koh, Jin Hyuck Heo, Mohammad Afsar Uddin, Young-Wan Kwon, Dong Hoon Choi, Sang Hyuk Im, Han Young Woo, ACS Appl. Mater. Interfaces 2017, 9, 50, 43846-43854.

[133] A high-mobility electron-transporting polymer for printed transistors, He Yan, Zhihua Chen, Yan Zheng, Christopher Newman, Jordan R. Quinn, 
Florian Dotz, Marcel Kastler and Antonio Facchetti, Nature volume 457, pages 679-686 (2009).

[134] Polymer Blend Solar Cells Based on a High-Mobility NaphthalenediimideBased Polymer Acceptor: Device Physics, Photophysics and Morphology, Jennifer R. Moore, Sebastian Albert-Seifried, Akshay Rao, Sylvain Massip, Benjamin Watts, David J. Morgan, Richard H. Friend, Christopher R. McNeill, and Henning Sirringhaus, Adv. Energy Mater. 2011, 1, 230-240.

[135] Comparing the device physics, dynamics and morphology of polymer solar cells employing conventional PCBM and non-fullerene polymer acceptor N2200, Jianyu Yuan, Wenping Guo, Yuxin Xia, Michael J. Ford, Feng Jin, Dongyang Liu, Haibin Zhao, Olle Inganäs, Guillermo C. Bazan, Wanli Ma, Nano Energy 35 (2017) 251-262.

[136] Key Parameters Requirements for Non-Fullerene-Based Organic Solar Cells with Power Conversion Efficiency 20\%, Yuliar Firdaus, Vincent M. Le Corre, Jafar I. Khan, Zhipeng Kan, Frédéric Laquai, Pierre M. Beaujuge, and Thomas D. Anthopoulos, Adv. Sci. 2019, 6, 1802028.

[137] Intrinsic non-radiative voltage losses in fullerene-based organic solar cells. Johannes Benduhn, Kristofer Tvingstedt, Fortunato Piersimoni, Sascha Ullbrich, Yeli Fan, Manuel Tropiano, Kathryn A. McGarry, Olaf Zeika, Moritz K. Riede, Christopher J. Douglas, Stephen Barlow, Seth R. Marder, Dieter Neher, Donato Spoltore and Koen Vandewal. Nat. Energy 2017, 2, $17053 .$.

[138] Hybridization of Local Exciton and Charge-Transfer States Reduces Nonradiative Voltage Losses in Organic Solar Cells. Flurin D. Eisner, Mohammed Azzouzi, Zhuping FeiXueyan Hou, Thomas D. Anthopoulos, T. John S. Dennis, Martin Heeney and Jenny Nelson. Am. Chem. Soc. 2019, 141, 15, 6362-6374. 
[139] A Close Look at Charge Generation in Polymer:Fullerene Blends with Microstructure Control, Mariateresa Scarongella, Jelissa De Jonghe-Risse, Ester Buchaca-Domingo, Martina Causa, Zhuping Fei, Martin Heeney, Jacques-E. Moser, Natalie Stingelin and Natalie Banerji. J. Am. Chem. Soc. 2015, 137, 2908-2918

[140] Effect of Annealing on P3HT:PCBM Charge Transfer and Nanoscale Morphology Probed by Ultrafast Spectroscopy, R. Alex Marsh, Justin M. Hodgkiss, Sebastian Albert-Seifried and Richard H. Friend, Nano Lett. 2010, 10, 923-930.

[141] Electronic Processes in Organic Semiconductors, Anna Köhler and Heinz Bässler, Print ISBN: 978-3-527-33292-2.

[142] Designing high performance all-small-molecule solar cells with nonfullerene acceptors: comprehensive studies on photoexcitation dynamics and charge separation kinetics, Junqing Shi, Anna Isakova, Abasi Abudulimu, Marius van den Berg, Oh Kyu Kwon, Alfred J. Meixner, Soo Young Park, Dai Zhang, Johannes Gierschner and Larry Lüer, Energy Environ. Sci., 2018,11, 211-220.

[143] Transient absorption spectroscopy of polymer-based thin-film solar cells. Hideo Ohkita, Shinzaburo Ito, Polymer 52 (2011) 4397-4417.

[144] Discerning Bulk and Interfacial Polarons in a Dual Electron Donor/Acceptor Polymer. Jose M. Marin-Beloqui, Kealan J. Fallon, Hugo Bronstein, and Tracey M. Clarke. J. Phys. Chem. Lett. 2019, 10, 3813-3819.

[145] Ultrafast Long-Range Charge Separation in Organic Semiconductor Photovoltaic Diodes. Simon Gélinas, Akshay Rao, Abhishek Kumar, Samuel L. Smith, Alex W. Chin, Jenny Clark, Tom S. van der Poll, Guillermo C. Bazan, Richard H. Friend. Science Vol. 343, Issue 6170, pp. 512-51. 
[146] The influence of microstructure on charge separation dynamics in organic bulk heterojunction materials for solar cell applications. M. Scarongella, A. A. Paraecattil, E. Buchaca-Domingo, J. D. Douglas,c S. Beaupré, T. McCarthyWard, M. Heeney, J.-E. Moser, M. Leclerc, J. M. J. Fréchet, N. Stingelinb and N. Banerji. J. Mater. Chem. A, 2014, 2, 6218.

[147] The fate of electron-hole pairs in polymer:fullerene blends for organic photovoltaics. Martina Causa, Jelissa De Jonghe-Risse, Mariateresa Scarongella, Jan C. Brauer, Ester Buchaca-Domingo, Jacques-E. Moser, Natalie Stingelin and Natalie Banerji. Nat Commun 7, 12556 (2016).

[148] The Effect of Solvent Additives on Morphology and Excited-State Dynamics in PCPDTBT:PCBM Photovoltaic Blends. Fabian Etzold, Ian A. Howard, Nina Forler, Don M. Cho, Michael Meister, Hannah Mangold, Jie Shu, Michael Ryan Hansen, Klaus Müllen, and Frédéric Laquai. J. Am. Chem. Soc. 2012, 134, 10569-10583.

[149] Pump-Probe Spectroscopy in Organic Semiconductors: Monitoring Fundamental Processes of Relevance in Optoelectronics, Juan CabanillasGonzalez, Giulia Grancini and Guglielmo Lanzani, Adv. Mater. 2011, 23, 54685485.

[150] Differential Evolution - a Simple and Efficient Heuristic for Global Optimization over Continuous Spaces, Storn R, and Price K., Journal of Global Optimization, 1997, 11, 341-359.

[151] Bimolecular recombination in polymer/fullerene bulk heterojunction solar cells, L. J. A. Koster, V. D. Mihailetchi, and P. W. M. Blom, Applied Physics Letters 88, 0521042006. 
[152] Bulk Heterojunction Photovoltaic Cells with Low Donor Concentration. Zhang, M., Wang, H., Tian, H., Geng, Y., Tang, C. W. Adv. Mater. 2011, 23, 4960-4964.

[153] Fullerene-Based Schottky-Junction Organic Solar Cells: A Brief Review. Sutty, S., Williams, G., Aziz, H. J. Photonics Energy 2014, 4, 040999.

[154] Highly Efficient Bulk Heterojunction Photovoltaic Cells Based on C70 and Tetraphenyldibenzoperiflanthene. Zheng, Y., Potscavage, W. J., Komino, T., Hirade, M., Adachi, J., Adachi, C. Appl. Phys. Lett. 2013, 102, 143304.

[155] Quantitative Analyses of Competing Photocurrent Generation Mechanisms in Fullerene-Based Organic Photovoltaics. Xu, L.; Wang, J.; Villa, M. de A.; Daunis, T. B.; Lee, Y.-J.; Malko, A. V.; Hsu, J. W. P. J. Phys. Chem. C 2016, 120, 16470-16477.

[156] Increased Open- Circuit Voltage of Organic Solar Cells by Reduced DonorAcceptor Interface Area. Vandewal, K.; Widmer, J.; Heumueller, T.; Brabec, C. J.; McGehee, M. D.; Leo, K.; Riede, M.; Salleo, A. Adv. Mater. 2014, 26, 3839-3843.

[157] Charge Transport in Pure and Mixed Phases in Organic Solar Cells. Melianas, A.; Pranculis, V.; Spoltore, D.; Benduhn, J.; Inganäs, O.; Gulbinas, V.; Vandewal, K.; Kemerink, M. Adv. Energy Mater. 2017, 7, 1700888.

[158] Origin of Photocurrent in Fullerene-Based Solar Cells. Albes, T.; Xu, L.; Wang, J.; Hsu, J. W. P.; Gagliardi, A. J. Phys. Chem. C 2018, 122, 15140-15148.

[159] Charge Transfer States in Dilute Donor-Acceptor Blend Organic Heterojunctions. Liu, X.; Ding, K.; Panda, A.; Forrest, S. R. ACS Nano 2016, 10, 76197626. 
[160] Absorption Tails of Donor:C 60 Blends Provide Insight into Thermally Activated Charge-Transfer Processes and Polaron Relaxation. Vandewal, K.; Benduhn, J.; Schellhammer, K. S.; Vangerven, T.; Ruückert, J. E.; Piersimoni, F; Scholz, R.; Zeika, O.; Fan, Y.; Barlow, S.; Neher, D.; Marder, S. R.; Manca, J.; Spoltore, D.; Cuniberti, G.; Ortmann, F. J. Am. Chem. Soc. 2017, 139, 16991704.

[161] Hole Transport in Low-Donor-Content Organic Solar Cells. Spoltore, D.; Hofacker, A.; Benduhn, J.; Ullbrich, S.; Nyman, M.; Zeika, O.; Schellhammer, S.; Fan, Y.; Ramirez, I.; Barlow, S.; Riede, M.; Marder, S. R.; Ortmann, F.; Vandewal, K. J. Phys. Chem. Lett. 2018, 9, 5496-5501.

[162] Loss Mechanisms in Fullerene-Based Low-Donor Content Organic Solar Cells. Jiang, W.; Jin, H.; Stolterfoht, M.; Shaw, P. E.; Nagiri, R. C. R.; Kopidakis, N.; Burn, P. L. J. Phys. Chem. C 2018, 122, 20611-20618.

[163] Femtosecond Dynamics of Photoexcited C60 Films. Martina Causa', Ivan Ramirez, Josue F. Martinez Hardigree, Moritz Riede, and Natalie Banerji. J. Phys. Chem. Lett. 2018, 9, 1885-1892.

[164] Femtosecond Dynamics of Photoexcited C60 Films. Martina Causa', Ivan Ramirez, Josue F. Martinez Hardigree, Moritz Riede, and Natalie Banerji. J. Phys. Chem. Lett. 2018, 9, 1885-1892.

[165] Charge transport in Pure and Mixed Phases in Organic Solar Cells. Melianas, A.; Pranculis, V.; Spoltore, D.; Benduhn, J.; Inganäs, O.; Gulbinas, V.; Vandewal, K.; Kemerink, M. Adv. Energy Mater. 2017, 7, 1700888.

[166] Effect of Polarization on the Mobility of C 60: A Kinetic Monte Carlo Study. Volpi, R.; Kottravel, S.; Nørby, M. S.; Stafström, S.; Linares, M. J. Chem. Theory Comput. 2016, 12, 812-824. 
[167] Design rules for minimizing voltage losses in high-efficiency organic solar cells. Deping Qian, Zilong Zheng, Huifeng Yao, Wolfgang Tress, Thomas R. Hopper, Shula Chen, Sunsun Li, Jing Liu, Shangshang Chen, Jiangbin Zhang, Xiao-Ke Liu, Bowei Gao, Liangqi Ouyang, Yingzhi Jin, Galia Pozina, Irina A. Buyanova, Weimin M. Chen, Olle Inganäs, Veaceslav Coropceanu, Jean-Luc Bredas, He Yan, Jianhui Hou, Fengling Zhang, Artem A. Bakulin and Feng Gao. Nature Materials volume 17, pages 703-709 (2018).

[168] Molecular Optimization Enables over 13\% Efficiency in Organic Solar Cells. Zhao, W.; Li, S.; Yao, H.; Zhang, S.; Zhang, Y.; Yang, B.; Hou, J. J. Am. Chem. Soc. $2017,139,7148-7151$. 